\title{
GLOBAL JOURNAL
}

OF SCIENCE FRONTIER RESEARCH: A

\section{Physics and Space Science}

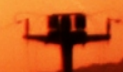

Dust Cosmological Models

Styrene Polymerization Kinetics

\section{Highlights}

\section{Explanation of Dark Matter}

Role of the Gailitis Resonance

Discovering Thoughts, Inventing Future VOLUME $15 \quad$ ISSUE 1 VERSION 1.0 
Global JOURnAl of SCIENCE Frontier ReseARCH: A PHYSICS \& SPACE SCIENCE 
Global Journal of SCIENCE Frontier ReSEARCH: A PHYSICS \& SPACE SCIENCE

VOLUME 15 ISSUE 1 (VER. 1.0) 
(C) Global Journal of Science

Frontier Research. 2015.

All rights reserved.

This is a special issue published in version 1.0 of "Global Journal of Science Frontier

Research.” By Global Journals Inc.

All articles are open access articles distributed under "Global Journal of Science Frontier Research"

Reading License, which permits restricted use. Entire contents are copyright by of "Global Journal of Science Frontier Research" unless otherwise noted on specific articles.

No part of this publication may be reproduced or transmitted in any form or by any means, electronic or mechanical, including photocopy, recording, or any information storage and retrieval system, without written permission.

The opinions and statements made in this book are those of the authors concerned.

Ultraculture has not verified and neither confirms nor denies any of the foregoing and no warranty or fitness is implied.

Engage with the contents herein at your own risk.

The use of this journal, and the terms and conditions for our providing information, is governed by our Disclaimer, Terms and Conditions and Privacy Policy given on our website http://globaljournals.us/terms-and-condition/ menu-id-1463/

By referring / using / reading / any type of association / referencing this journal, this signifies and you acknowledge that you have read them and that you accept and will be bound by the terms thereof.

All information, journals, this journal, activities undertaken, materials, services and our website, terms and conditions, privacy policy, and this journal is subject to change anytime without any prior notice.

Incorporation No.: 0423089

License No.: 42125/022010/1186

Registration No.: 430374

Import-Export Code: 1109007027 Employer Identification Number (EIN): USA Tax ID: 98-0673427

\section{Global Journals Inc.}

(A Delaware USA Incorporation with "Good Standing”; Reg. Number: 0423089) Sponsors: Open Association of Research Society

Open Scientific Standards

\section{Publisher's Headquarters office}

Global Journals Headquarters

301st Edgewater Place Suite, 100 Edgewater Dr.-Pl, Wakefield MASSACHUSETTS, Pin: 01880, United States of America

USA Toll Free: +001-888-839-7392

USA Toll Free Fax: +001-888-839-7392

\section{Offset Typesetting}

Global Journals Incorporated

2nd, Lansdowne, Lansdowne Rd., Croydon-Surrey, Pin: CR9 2ER, United Kingdom

\section{Packaging \& Continental Dispatching}

Global Journals

E-3130 Sudama Nagar, Near Gopur Square, Indore, M.P., Pin:452009, India

Find a correspondence nodal officer near you

To find nodal officer of your country, please email us atlocal@globaljournals.org

eContacts

Press Inquiries: press@globaljournals.org Investor Inquiries: investors@globaljournals.org Technical Support: technology@globaljournals.org Media \& Releases: media@globaljournals.org

Pricing (Including by Air Parcel Charges):

For Authors:

22 USD (B/W) \& 50 USD (Color)

Yearly Subscription (Personal \& Institutional): 200 USD (B/W) \& 250 USD (Color) 
John A. Hamilton," Drew" Jr.,

Ph.D., Professor, Management

Computer Science and Software

Engineering

Director, Information Assurance

Laboratory

Auburn University

\section{Dr. Henry Hexmoor}

IEEE senior member since 2004

Ph.D. Computer Science, University at

Buffalo

Department of Computer Science

Southern Illinois University at Carbondale

\section{Dr. Osman Balci, Professor}

Department of Computer Science

Virginia Tech, Virginia University

Ph.D.and M.S.Syracuse University,

Syracuse, New York

M.S. and B.S. Bogazici University, Istanbul, Turkey

\section{Yogita Bajpai}

M.Sc. (Computer Science), FICCT

U.S.A.Email:

yogita@computerresearch.org

\section{Dr. T. David A. Forbes}

Associate Professor and Range

Nutritionist

Ph.D. Edinburgh University - Animal

Nutrition

M.S. Aberdeen University - Animal

Nutrition

B.A. University of Dublin- Zoology

\section{Dr. Wenying Feng}

Professor, Department of Computing \& Information Systems

Department of Mathematics

Trent University, Peterborough,

ON Canada K9J 7B8

\section{Dr. Thomas Wischgoll}

Computer Science and Engineering, Wright State University, Dayton, Ohio

B.S., M.S., Ph.D.

(University of Kaiserslautern)

Dr. Abdurrahman Arslanyilmaz

Computer Science \& Information Systems

Department

Youngstown State University

Ph.D., Texas A\&M University

University of Missouri, Columbia

Gazi University, Turkey

\section{Dr. Xiaohong He}

Professor of International Business

University of Quinnipiac

BS, Jilin Institute of Technology; MA, MS,

$\mathrm{PhD}$, . (University of Texas-Dallas)

\section{Burcin Becerik-Gerber}

University of Southern California

Ph.D. in Civil Engineering

DDes from Harvard University

M.S. from University of California, Berkeley

\& Istanbul University 


\begin{abstract}
Dr. Bart Lambrecht
Director of Research in Accounting and

FinanceProfessor of Finance

Lancaster University Management School

BA (Antwerp); MPhil, MA, PhD
\end{abstract}

(Cambridge)

\section{Dr. Carlos García Pont}

Associate Professor of Marketing

IESE Business School, University of

Navarra

Doctor of Philosophy (Management), Massachusetts Institute of Technology (MIT)

Master in Business Administration, IESE, University of Navarra

Degree in Industrial Engineering, Universitat Politècnica de Catalunya

\section{Dr. Fotini Labropulu}

Mathematics - Luther College

University of ReginaPh.D., M.Sc. in

Mathematics

B.A. (Honors) in Mathematics

University of Windso

\section{Dr. Lynn Lim}

Reader in Business and Marketing

Roehampton University, London

BCom, PGDip, MBA (Distinction), PhD, FHEA

\section{Dr. Mihaly Mezei}

ASSOCIATE PROFESSOR

Department of Structural and Chemical Biology, Mount Sinai School of Medical Center

Ph.D., Etvs Lornd University

Postdoctoral Training,

New York University

\section{Dr. Söhnke M. Bartram}

Department of Accounting and

FinanceLancaster University Management

SchoolPh.D. (WHU Koblenz)

MBA/BBA (University of Saarbrücken)

\section{Dr. Miguel Angel Ariño}

Professor of Decision Sciences

IESE Business School

Barcelona, Spain (Universidad de Navarra) CEIBS (China Europe International Business School).

Beijing, Shanghai and Shenzhen

Ph.D. in Mathematics

University of Barcelona

BA in Mathematics (Licenciatura)

University of Barcelona

\section{Philip G. Moscoso}

Technology and Operations Management IESE Business School, University of Navarra Ph.D in Industrial Engineering and

Management, ETH Zurich

M.Sc. in Chemical Engineering, ETH Zurich

Dr. Sanjay Dixit, M.D.

Director, EP Laboratories, Philadelphia VA Medical Center

Cardiovascular Medicine - Cardiac

Arrhythmia

Univ of Penn School of Medicine

\section{Dr. Han-Xiang Deng}

MD., Ph.D

Associate Professor and Research

Department Division of Neuromuscular Medicine

Davee Department of Neurology and Clinical NeuroscienceNorthwestern University Feinberg School of Medicine 


\section{Dr. Pina C. Sanelli}

Associate Professor of Public Health

Weill Cornell Medical College

Associate Attending Radiologist

NewYork-Presbyterian Hospital

MRI, MRA, CT, and CTA

Neuroradiology and Diagnostic

Radiology

M.D., State University of New York at

Buffalo,School of Medicine and

Biomedical Sciences

\section{Dr. Roberto Sanchez}

Associate Professor

Department of Structural and Chemical

Biology

Mount Sinai School of Medicine

Ph.D., The Rockefeller University

\section{Dr. Wen-Yih Sun}

Professor of Earth and Atmospheric

SciencesPurdue University Director

National Center for Typhoon and

Flooding Research, Taiwan

University Chair Professor

Department of Atmospheric Sciences,

National Central University, Chung-Li,

TaiwanUniversity Chair Professor

Institute of Environmental Engineering,

National Chiao Tung University, Hsinchu, Taiwan.Ph.D., MS The University of

Chicago, Geophysical Sciences

BS National Taiwan University,

Atmospheric Sciences

Associate Professor of Radiology

\section{Dr. Michael R. Rudnick}

M.D., FACP

Associate Professor of Medicine

Chief, Renal Electrolyte and

Hypertension Division (PMC)

Penn Medicine, University of

Pennsylvania

Presbyterian Medical Center,

Philadelphia

Nephrology and Internal Medicine

Certified by the American Board of Internal Medicine

\section{Dr. Bassey Benjamin Esu}

B.Sc. Marketing; MBA Marketing; Ph.D

Marketing

Lecturer, Department of Marketing, University of Calabar

Tourism Consultant, Cross River State

Tourism Development Department

Co-ordinator , Sustainable Tourism

Initiative, Calabar, Nigeria

Dr. Aziz M. Barbar, Ph.D.

IEEE Senior Member

Chairperson, Department of Computer

Science

AUST - American University of Science \& Technology

Alfred Naccash Avenue - Ashrafieh 
PRESIDENT EDITOR (HON.)

Dr. George Perry, (Neuroscientist)

Dean and Professor, College of Sciences

Denham Harman Research Award (American Aging Association)

ISI Highly Cited Researcher, Iberoamerican Molecular Biology Organization

AAAS Fellow, Correspondent Member of Spanish Royal Academy of Sciences

University of Texas at San Antonio

Postdoctoral Fellow (Department of Cell Biology)

Baylor College of Medicine

Houston, Texas, United States

Chief Author (HON.)

\section{Dr. R.K. Dixit}

M.Sc., Ph.D., FICCT

Chief Author, India

Email: authorind@computerresearch.org

\section{DEAN \& EDITOR-IN-CHIEF (HON.)}

\section{Vivek Dubey(HON.)}

MS (Industrial Engineering),

MS (Mechanical Engineering)

University of Wisconsin, FICCT

Editor-in-Chief, USA

editorusa@computerresearch.org

\section{Sangita Dixit}

M.Sc., FICCT

Dean \& Chancellor (Asia Pacific) deanind@computerresearch.org

\section{Suyash Dixit}

(B.E., Computer Science Engineering), FICCTT President, Web Administration and Development , CEO at IOSRD COO at GAOR \& OSS

\section{Er. Suyog Dixit}

(M. Tech), BE (HONS. in CSE), FICCT

SAP Certified Consultant

CEO at IOSRD, GAOR \& OSS

Technical Dean, Global Journals Inc. (US)

Website: www.suyogdixit.com

Email:suyog@suyogdixit.com

\section{Pritesh Rajvaidya}

(MS) Computer Science Department

California State University

BE (Computer Science), FICCT

Technical Dean, USA

Email: pritesh@computerresearch.org

Luis Galárraga

J!Research Project Leader

Saarbrücken, Germany 


\section{CONTENTS OF THE ISSUE}

i. Copyright Notice

ii. Editorial Board Members

iii. Chief Author and Dean

iv. Contents of the Issue

1. Some Dust Cosmological Models with Time Dependent $\Lambda(T)$ in Creation Field Cosmology. 1-11

2. Explanation of Dark Matter and Dark Energy Phenomena. 13-18

3. Possible Role of the Gailitis Resonance in Low Energy Nuclear Fusion Experiments. 19-23

4. Formulate Equations for Reciprocity Vector and Scalar of Some Mechanical and Electrical Quantities. 25-34

5. Factor Analysis of the Styrene Polymerization Kinetics Statistical Verification of the Procedure. 35-39

6. Is Spacetime Fractal and Quantum Coherent in the Golden Mean? 41-60

v. Fellows and Auxiliary Memberships

vi. Process of Submission of Research Paper

vii. Preferred Author Guidelines

viii. Index 


\title{
Some Dust Cosmological Models with Time Dependent $\Lambda(t)$ in Creation Field Cosmology
}

\author{
By H. R. Ghate \& Sanjay A. Salve \\ Jijamata Mahavidyalaya Buldana, India
}

Abstract-We have studied the Hoyle-Narlikar's Creation-field cosmology for LRS Bianchi type-II, LRS Bianchi type- $\mathrm{VI}_{0}$, Plane Symmetric and Kantowski-Sachs universes with time dependent cosmological constant $\Lambda(t)$, when the universe is filled with dust distribution. To get deterministic model of the universe, a relation between shear $(\sigma)$ and expansion $(\theta)$ is assumed. The physical aspects of the models are also discussed.

Keywords: Creation-field cosmology, Cosmological constant $\Lambda(t)$.

GJSFR-A Classification : FOR Code: 020109

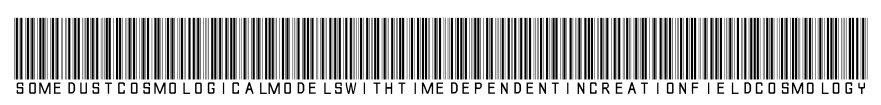

Strictly as per the compliance and regulations of:

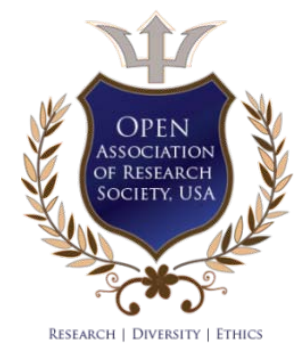

(c) 2015. H. R. Ghate \& Sanjay A. Salve. This is a research/review paper, distributed under the terms of the Creative Commons Attribution-Noncommercial 3.0 Unported License http://creativecommons.org/licenses/by-nc/3.0/), permitting all non commercial use, distribution, and reproduction in any medium, provided the original work is properly cited. 


\title{
Some Dust Cosmological Models with Time Dependent $\wedge(t)$ in Creation Field Cosmology
}

\author{
H. R. Ghate ${ }^{\alpha}$ \& Sanjay A. Salve ${ }^{\sigma}$
}

Abstract- We have studied the Hoyle-Narlikar's Creation-field cosmology for LRS Bianchi type-II, LRS Bianchi type- $\mathrm{VI}_{0}$, Plane Symmetric and Kantowski-Sachs universes with time dependent cosmological constant $\Lambda(t)$, when the universe is filled with dust distribution. To get deterministic model of the universe, a relation between shear $(\sigma)$ and expansion $(\theta)$ is assumed. The physical aspects of the models are also discussed.

Keywords: Creation-field cosmology, Cosmological constant $\Lambda(t)$

\section{InTRODUCTION}

$(1$ osmology is the scientific study of origin, evolution, large scale structures \& dynamics of the universe. It involves the formation of theories or hypothesis about the universe which makes specific predictions for phenomenon. These predictions can be tested with observations. Einstein's theory of general relativity is a very successful gravitational theory in describing the gravitational phenomena which also served as a basis for the models of the universe. All the investigations dealing with physical process are successfully explained by Einstein's field equations based big-bang model. The phenomenon of expanding universe, Primordial nucleo synthesis \& the observed isotropy of Comic Microwave Background Radiations (CMBR) are important observations in astronomy which were successfully explained the big-bang model. This model is described by FRW line element and a matter density source which obeys equation of state $\rho=3 p$, where $p$ and $\rho$ are the fluid pressure and matter density respectively. But this model has various problems like; singularity in the past and may possibly in the future; no remarkable predictions in the big-bang model that explain the origin, evolution and characteristic of structures in the universe; the conservation of the energy is violated; the flatness and horizon problem explanations given from big-bang model of the universe.

However Smoot et al. [1] revealed that the astronomical predictions of the FRW type of models do not always exactly meet our expectations. The

\footnotetext{
Author a: Department of Mathematics, Jijamata Mahavidyalaya,Buldana (M.S.) India.e-mail: hrghate@gmail.com Author o: Department of Mathematics, Shri Shivaji Science and Arts College, Chikhli, Dist. Buldana (M.S.) India.

e-mail:salvesanjaya@gmail.com
}

theoretical explanations given from big -bang type model were contradicted by some puzzling results regarding the red-shifts from extra-galactic objects. The gravitational collapse of massive objects is an unavoidable consequence of general relativity [2-3]. Also CMBR discovery did not prove it to be an outcome of big-bang theory. Therefore alternative theories of gravitation were proposed time to time to overcome the drawbacks of big-bang model. Bondi \& Gold [4] proposed a steady state theory in which the universe does not have any singular beginning or an end on the cosmic time scale where the matter density is throughout constant. Also they state that the statistical properties of the large scale features of the universe do not change. Further the constancy of mass density has been accounted by continuous creation of matter going on in contrast to the one time infinite and explosive creation of matter at $t=0$ as in earlier standard model. But the principle of conservation of matter was violated in this formalism. This difficulty was overcome by Hoyle \& Narlikar [5-7] by adopting a field theoretical approach and introducing a massless \& chargeless scalar field C in the Einstein-Hilbert action to explain creation of matter. In Hoyle-Narlikar C-field theory there is no bigbang type singularity as in steady state theory by Bondi \& Gold. Narlikar [8] has shown that the matter creation is accomplished at the expense of negative energy Cfield in which he solves horizon and flatness problem faced by big-bang model. Narlikar \& Padmanabhan [9] have obtained a solution of Einstein field equations admitting radiation with a negative energy massless scalar field C. In fact Narlikar et al. [10] have proved the possibilities of non-relic interpretation of CMBR. Chatterjee \& Banerjee [11] have investigated higher dimensional cosmology in C-field theory. Singh \& Chaubey [12] have studied Bianchi type I, III, V, VI $\mathrm{I}_{0}$ and Kantowski-Sachs universes in C-field Cosmology. Adhav et al. [13-14] have investigated higher dimensional Bianchi Type $\mathrm{VI}_{0}$ and Bianchi type-I string cosmological models in Creation field cosmology. Katore [15] has studied plane symmetric universe in C-field cosmology. Recently Patil et al. [16] have obtained Bianchi type-IX dust filled universe with ideal fluid distribution in Creation field theory.

Einstein's basic cosmological model was a static, homogeneous with spherical geometry. Since at that time universe was not known to be expanding, it is considered as the gravitational effect of matter caused 
acceleration in the model. In 1917, Einstein introduced a cosmological constant $\Lambda$ in his equation as the universal repulsion or anti-gravity effect to make the universe static in accordance with generally accepted picture of that time. Hubble showed that the universe was expanding by his study about nearby galaxies. Then Einstein regretted modifying his elegant theory and viewed the cosmological constant as his greatest mistake. Recent cosmological observations by the HighZ Supernovae Team and Supernovae Cosmological Project [17-25] suggest the existence of a positive cosmological constant $\Lambda$ with the magnitude $\Lambda\left(G \hbar / c^{3}\right) \approx 10^{-123}$. Zel'dovich [26] has tried to visualize the meaning of cosmological constant from the theory of elementary particles. Bergmann [27] has interpreted the cosmological constant $\Lambda$ in terms of Higgs scalar field. In quantum field theory, the cosmological constant is considered as the vacuum energy density. Linde [28] had shown that the cosmological term arises from spontaneous symmetry breaking and also suggested that the cosmological term is not a constant but a function of temperature. Dolgo has [29-30] focused that cosmological constant remains constant in the absence of any interaction with matter and radiation. Pavon [31] have studied model with cosmological constant problem as the discrepancy between the negligible value for the present universe. Glashow-Salam-Weinberg model [33] expected $10^{50}$ larger value whereas grand unified theory [34] expected $10^{107}$ larger value whereas of cosmological constant. Bertolami [35] was the first who consider cosmological models with a variable cosmological constant of the form $\Lambda \sim t^{-2}$. Berman and Som [36] have shown that $\Lambda \sim t^{-2}$ plays a very important role in cosmology. Chen and Wu [37] have also solved the problem by considering $\Lambda \sim R^{-2}$, where $R$ is the scale factor in the Robertson-Walker space time. Carvalho et al. [38] generalized the proposed model of Chen and Wu by including a term proportional to $\mathrm{H}^{2}$ on the time dependence of $\Lambda$. Overduin [39] considered the assumptions $\Lambda \sim t^{-2}$ and $\Lambda \sim H^{-2}$ in FRW models and shows there compability with various observations. At the same time cosmologist viz. Olson et al. [40], Gasperini [41], Pebbles \& Ratra [42], Abdel Rahman [43], Maia et al. [44], Silveira et al. [45], Moffat [46], Torres et al. [47], Abdussattar \& Vishwakarma [48], Hoyle et al. [49], Podariu et al. [50] and many other authors had shown that cosmological term decays with time. Carmeli \& Kuzmenko [51] have shown that cosmological relativistic theory predicts the value of cosmological constant $\Lambda=1.934 \times 10^{-35} \mathrm{~s}^{-2}$. Recent observations indicate that $\Lambda \sim 10^{-56} \mathrm{~cm}^{-2}$ but the theory of physics of elementary particles predicts that the value of $\Lambda$ must have been $10^{120}$ times larger in the past. It is worth noting that cosmological models based on Einstein field equations with a time-dependent cosmological constant $\Lambda$ had been the subject of numerous papers in recent years. Several attempts have made by many researchers viz. Lui \& Wesson [52], Cunha et al. [53], Carneiro et al. [54], Pradhan et al. [55], Singh et al. [56], Katore et al. [57], Tiwari et al.[58], Dwivedi [59] and Amirhashchi \& Mohamadian [60] in the favor of time dependent $\Lambda \sim t^{-2}$ in different contexts. Bali et al. [61-62] have investigated Bianchi type III \& FRW cosmological models with varying $\Lambda$ in Creation field cosmology. Rahman \& Ansari [63] obtained some new LRS Bianchi type-I bulk viscous cosmological models with decaying $\Lambda$-term. Recently Ghate et al. [64-66] have studied cosmological models with varying $\Lambda(t)$ in creation field theory of gravitation.

In this paper, we have investigated LRS Bianchi type-II (LRS B-II), LRS Bianchi type-VI $($ LRS B-VI $)$, Plane Symmetric (PS) and Kantowsaki-Sachs (K-S) spacetimes with varying cosmological constant $\Lambda(t)$ in creation field theory of gravitation. To obtain solution, a relation between $\operatorname{shear}(\sigma)$ and $\operatorname{expansion}(\theta)$ is assumed. This work is organized as follows. In Section 2. Hoyle-Narlikar creation field theory is briefly discussed. An exact solution of field equations for LRS B-II, LRS B-VI, PS \& K-S spaces-times have been obtained in section 3, 4, 5 \& 6 respectively. The physical aspects of the anisotropic models have been discussed in section 7, while in Section 8, concluding remarks have been expressed.

\section{il. Hoyle-Narlikar Theory}

Hoyle and Narlikar [5-7] have modified Einstein field equations through the introduction of a massless scalar field usually called Creation field viz. $C$-field. The modified field equations are

$$
R_{i}^{j}-\frac{1}{2} R g_{i}^{j}=-8 \pi G\left[\begin{array}{cc}
T_{i}^{j}+T_{i}^{j} \\
(m) & (c)
\end{array}\right]-\Lambda(t) g_{i}^{j},
$$

where $T_{i}^{j}$ is a matter tensor for perfect fluid of Einstein's theory given by

$$
\underset{(m)}{T_{i}^{j}}=(\rho+p) v_{i} v^{j}-p g_{i}^{j}
$$

and $T_{i}^{j}$ is a matter tensor due to $C$-field given by (c)

$$
\underset{\substack{(c) \\ T^{j}}}{ }=-f\left(C_{i} C^{j}-\frac{1}{2} g_{i}^{j} C^{\alpha} C_{\alpha}\right) .
$$

Here $\rho$ is the energy density of massive particles and $p$ is the pressure. $v_{i}$ are co-moving four 
velocities which obeys the relation $v_{i} v^{j}=1, v_{\alpha}=0$, $\alpha=1,2,3 . f>0$ is the coupling constant between matter and creation field and $C_{i}=\frac{d C}{d x^{i}}$.

As $T^{00}$ has negative value (i.e. $\left.T^{00}<0\right)$, the $C$ field has negative energy density producing repulsive gravitational field which causes the expansion of the universe. Thus the energy conservation law reduces to

$$
{ }^{(m)} T_{; j}^{i j}=-{ }^{(c)} T_{; j}^{i j}=f C^{i} C^{j} ; j,
$$

i.e. the matter creation through a non-zero left hand side is possible while conserving the overall energy and momentum.

The above equation is identical with

$$
m g_{i j} \frac{d x^{i}}{d s}-C_{j}=0
$$

which indicates the 4-momentum of the created particle is compensated by 4-momentum of the C-field. In order to maintain the balance, the $\mathrm{C}$-field must have negative energy.

Further the C-field satisfies the source equation

$$
f C_{; i}^{i}=J_{; i}^{i} \text { and } \quad J^{i}=\rho \frac{d x^{i}}{d s}=\rho v^{i}
$$

where $\rho$ is the homogeneous mass density.

The conservation equation for $\mathrm{C}$-field is given by

$$
\left(8 \pi G T_{i}^{j}+\Lambda g_{i}^{j}\right)_{; j}=0 \text {. }
$$

The physical quantities in cosmology are the mean anisotropy parameter $(\Delta)$ and the deceleration parameter $(q)$ are defined as

$$
\begin{aligned}
\Delta & =\frac{1}{3} \sum_{i=1}^{3}\left(\frac{H_{i}-H}{H}\right)^{2}, \\
q & =-\frac{R \ddot{R}}{\dot{R}^{2}}
\end{aligned}
$$

where $H$ is Hubble parameter.

\section{ili. LRS Bianchi Type-II Model}

We consider homogeneous LRS Bianchi-II metric in the form of

$$
d s^{2}=d t^{2}-A^{2} d x^{2}-B^{2} d y^{2}-2 B^{2} x d y d z-\left(B^{2} x^{2}+A^{2}\right) d z^{2}
$$

where metric potentials $A, B$ are functions of time $t$ only and $\sqrt{-g}=A^{2} B$.
It is assumed that Creation field $C$ is a function of time $t$ only i.e.

$$
C(x, t)=C(t) \text { and }{ }^{m} T_{j}^{i}=\operatorname{diag}(\rho,-p,-p,-p)
$$

The Hoyle-Narlikar field equations (2.1) with the help of equations (2.2) and (2.3) for the metric (3.1) given by

$$
\begin{aligned}
& \frac{\dot{A}^{2}}{A^{2}}+2 \frac{\dot{A} \dot{B}}{A B}-\frac{1}{4} \frac{B^{2}}{A^{4}}=8 \pi G\left(\rho-\frac{1}{2} f \dot{C}^{2}\right)+\Lambda, \\
& \frac{\ddot{A}}{A}+\frac{\ddot{B}}{B}+\frac{\dot{A} \dot{B}}{A B}+\frac{1}{4} \frac{B^{2}}{A^{4}}=8 \pi G\left(-p+\frac{1}{2} f \dot{C}^{2}\right)+\Lambda, \\
& 2 \frac{\ddot{A}}{A}+\frac{\dot{A}^{2}}{A^{2}}-\frac{3}{4} \frac{B^{2}}{A^{4}}=8 \pi G\left(-p+\frac{1}{2} f \dot{C}^{2}\right)+\Lambda,
\end{aligned}
$$

where overhead $\operatorname{dot}(\cdot)$ denotes differentiation with respect to time $t$.

The conservation equation (2.7) leads to

$$
\frac{d}{d t} \dot{C}^{2}+2\left(2 \frac{\dot{A}}{A}+\frac{\dot{B}}{B}\right) \dot{C}^{2}=\frac{2 \dot{\rho}}{f}+\frac{2 \rho}{f}\left(2 \frac{\dot{A}}{A}+\frac{\dot{B}}{B}\right)+\frac{\dot{\Lambda}}{4 \pi G f}
$$

where $p$ being isotropic pressure.

Following Hoyle and Narlikar, we have taken $p=0$, for the dust distribution. The source equation of $C$-field $C_{; i}^{j}=\frac{n}{f}$ leads to $C=t$ for large r. Thus $\dot{C}=1$.

Using $p=0 \& \dot{C}=1$, equations (3.3)-(3.5) lead to

$$
\begin{aligned}
& \frac{\dot{A}^{2}}{A^{2}}+2 \frac{\dot{A} \dot{B}}{A B}-\frac{1}{4} \frac{B^{2}}{A^{4}}=8 \pi G \rho-4 \pi G f+\Lambda, \\
& \frac{\ddot{A}}{A}+\frac{\ddot{B}}{B}+\frac{\dot{A} \dot{B}}{A B}+\frac{1}{4} \frac{B^{2}}{A^{4}}=4 \pi G f+\Lambda, \\
& 2 \frac{\ddot{A}}{A}+\frac{\dot{A}^{2}}{A^{2}}-\frac{3}{4} \frac{B^{2}}{A^{4}}=4 \pi G f+\Lambda .
\end{aligned}
$$

The field equations (3.7)-(3.9) is a system of three equations with four unknown parameters $A, B, \rho$ and $\Lambda$. To find the deterministic solution of the field equations, we need one extra condition. We assume that the shear scalar $(\sigma)$ is proportional to expansion scalar $(\theta)$ which leads to (Collins et al. [67])

$$
B=A^{n},
$$

where $n$ is arbitrary constant. 
From equations (3.8) and (3.9), we get

$$
\frac{\ddot{A}}{A}-\frac{\ddot{B}}{B}-\frac{\dot{A} \dot{B}}{A B}+\frac{\dot{A}^{2}}{A^{2}}-\frac{B^{2}}{A^{4}}=0 .
$$

Using equations (3.10) and (3.11), we have

$$
2 \ddot{A}+2(n+1) \frac{\dot{A}^{2}}{A}=\frac{2}{(1-n)} A^{2 n-3} .
$$

To get the deterministic value of $A$, Let $\dot{A}=F(A)$.

which implies $\ddot{A}=F F^{\prime}$, where $F^{\prime}=\frac{d F}{d A}$. reduces to

With the help of equation (3.13), equation (3.12)

$$
\frac{d F^{2}}{d A}+\frac{2(n+1)}{A} F^{2}=\frac{2}{(1-n)} A^{2 n-3} .
$$

Integrating equation (3.14), we get

$$
F^{2}=\frac{1}{2 n(1-n)} A^{2(n-1)}=\left(\frac{d A}{d t}\right)^{2} .
$$

The constant of integration has taken to be zero for simplicity.

Using $\dot{A}=F(A)$ and solving equation (3.15), we get

$$
A^{2-n}=\frac{(2-n)}{\sqrt{2 n(1-n)}} t+(2-n) C_{2},
$$

where $C_{2}$ is a constant of integration.

Simplifying equation (3.16), we get

$$
A=(a t+b)^{\frac{1}{2-n}}
$$

where $a=\frac{(2-n)}{\sqrt{2 n(1-n)}}, b=(2-n) C_{2}$ and $0<n<1$.

Using equation (3.17), equation (3.10) leads to

$$
B=(a t+b)^{\frac{n}{2-n}} \text {. }
$$

Substituting equations (3.17) \& (3.18) in equation (3.9), we have

$$
\Lambda=\frac{-4 a^{2}+8 n a^{2}-3 n^{2}+12 n-12}{4(2-n)^{2}(a t+b)^{2}}-k,
$$

where $4 \pi G f=k$.

Using equations (3.17), (3.18) and (3.19) in equation (3.7), we get

$$
8 \pi G \rho=\frac{4 a^{2}+n^{2}-4 n+4}{2(2-n)(a t+b)^{2}}+2 k,
$$

as $\frac{1}{a^{2}}=2 n\left(\frac{(1-n)}{(2-n)^{2}}\right)$.
Thus using equations (3.17) \& (3.18) in metric (3.1), the cosmological model is given by

$$
d s^{2}=d t^{2}-(a t+b)^{\frac{2}{2-n}} d x^{2}-(a t+b)^{\frac{2 n}{2-n}} d y^{2}-
$$

$$
2(a t+b)^{\frac{2 n}{2-n}} x d y d z-\left[(a t+b)^{\frac{2 n}{2-n}} x^{2}+(a t+b)^{\frac{2}{2-n}}\right] d z^{2} .
$$

Using equations (3.17), (3.18), (3.19) and (3.20) in equation (3.6), we have

$\frac{d}{d t} \dot{C}^{2}+\left[2\left(\frac{2+n}{2-n}\right)\left(\frac{a}{a t+b}\right)\right] \dot{C}^{2}=\left[2\left(\frac{2+n}{2-n}\right)\left(\frac{a}{a t+b}\right)\right]$

On integration, equation (3.22) reduces to

$$
\dot{C}=1 \text {, }
$$

which leads to

$$
C=t+b_{1},
$$

where $b_{1}$ is a constant of integration.

The value $\dot{C}=1$, So obtained agrees with the value used in the source equation. Thus Creation field $C$ is proportional to time $t$.

The physical parameters such as the spatial volume $(V)$, the mean anisotropic parameter $(\Delta)$ and the deceleration parameter $(q)$ are defined as

$$
\begin{aligned}
& V=(a t+b)^{\frac{2+n}{2-n}}, \\
& \Delta=2\left(\frac{1-n}{2+n}\right)^{2}=\text { Constant, }
\end{aligned}
$$

$$
q=-4 \frac{(n-1)}{(n+2)}=\text { Constant, where } 0<n<1 .
$$

Here $q>0$, for $0<n<1$.

\section{LRS Bianchi Trpe-VI 0 Model} the form of

We consider the LRS Bianchi type $\mathrm{VI}_{0}$ metric in

$$
d s^{2}=d t^{2}-A^{2} d x^{2}-B^{2}\left(e^{-2 x} d y^{2}+e^{2 x} d z^{2}\right)
$$

where metric potentials $A, B$ are functions of time $t$ only and $\sqrt{-g}=A B^{2}$.

It is assumed that Creation field $C$ is a function of time $t$ only

i.e. $C(x, t)=C(t)$ and ${ }^{m} T_{j}^{i}=\operatorname{diag}(\rho,-p,-p,-p)$ (4.2) 
The Hoyle-Narlikar field equations (2.1), with the help of equations (2.2) and (2.3) for the metric (4.1) given by

$$
\begin{aligned}
& \frac{\dot{B}^{2}}{B^{2}}+2 \frac{\dot{A} \dot{B}}{A B}-\frac{1}{A^{2}}=8 \pi G\left(\rho-\frac{1}{2} f \dot{C}^{2}\right)+\Lambda, \\
& 2 \frac{\ddot{B}}{B}+\frac{\dot{B}^{2}}{B^{2}}+\frac{1}{A^{2}}=8 \pi G\left(-p+\frac{1}{2} f \dot{C}^{2}\right)+\Lambda, \\
& \frac{\ddot{A}}{A}+\frac{\ddot{B}}{B}+\frac{\dot{A} \dot{B}}{A B}-\frac{1}{A^{2}}=8 \pi G\left(-p+\frac{1}{2} f \dot{C}^{2}\right)+\Lambda,
\end{aligned}
$$

where overhead do $t(\cdot)$ denotes differentiation with respect to time $t$.

The conservation equation (2.7) leads to

$$
\frac{d}{d t} \dot{C}^{2}+2\left(\frac{\dot{A}}{A}+2 \frac{\dot{B}}{B}\right) \dot{C}^{2}=\frac{2 \dot{\rho}}{f}+\frac{2 \rho}{f}\left(\frac{\dot{A}}{A}+2 \frac{\dot{B}}{B}\right)+\frac{\dot{\Lambda}}{4 \pi G f}
$$

where $p$ being isotropic pressure.

Following Hoyle and Narlikar, we have taken $p=0$, for the dust distribution. The source equation of $C$-field $C_{; i}^{j}=\frac{n}{f}$ leads to $C=t$ for large $r$. Thus $\dot{C}=1$. Using $p=0 \& \dot{C}=1$, equations (4.3)-(4.5) lead to

$$
\begin{aligned}
& \frac{\dot{B}^{2}}{B^{2}}+2 \frac{\dot{A} \dot{B}}{A B}-\frac{1}{A^{2}}=8 \pi G \rho-4 \pi G f+\Lambda, \\
& 2 \frac{\ddot{B}}{B}+\frac{\dot{B}^{2}}{B^{2}}+\frac{1}{A^{2}}=4 \pi G f+\Lambda, \\
& \frac{\ddot{A}}{A}+\frac{\ddot{B}}{B}+\frac{\dot{A} \dot{B}}{A B}-\frac{1}{A^{2}}=4 \pi G f+\Lambda .
\end{aligned}
$$

The field equations (4.7)-(4.9) is a system of three equations with four unknown parameters $A, B, \rho$ and $\Lambda$. To find the deterministic solution of the field equations, we need one extra condition. We assume that the shear scalar $(\sigma)$ is proportional to expansion scalar $(\theta)$ which leads to (Collins et al. [67])

$$
A=B^{n},
$$

where $n$ is arbitrary constant.

From equations (4.8) and (4.9), we get

$$
\frac{\ddot{A}}{A}-\frac{\ddot{B}}{B}+\frac{\dot{A} \dot{B}}{A B}-\frac{\dot{B}^{2}}{B^{2}}-\frac{2}{A^{2}}=0 .
$$

Using equations (4.10) and (4.11), we have

$$
2 \ddot{B}+2(n+1) \frac{\dot{B}^{2}}{B}=\frac{4}{(n-1)} B^{1-2 n}
$$

To get the deterministic value of $B$, Let $\dot{B}=F(B)$,

which implies $\ddot{B}=F F^{\prime}$, where $F^{\prime}=\frac{d F}{d B}$. reduces to

With the help of equation (4.13), equation (4.12)

$$
\frac{d F^{2}}{d B}+\frac{2(n+1)}{B} F^{2}=\frac{4}{(n-1)} B^{1-2 n} .
$$

Integrating equation (4.14), we get

$$
F^{2}=\frac{1}{(n-1)} B^{2(1-n)}=\left(\frac{d B}{d t}\right)^{2} .
$$

The constant of integration has taken to be zero for simplicity.

Using $\dot{B}=F(B)$ and solving equation (4.15), we get

$$
B^{n}=\frac{n}{\sqrt{n-1}} t+n C_{2}
$$

where $C_{2}$ is constant of integration.

Simplifying equation (4.16), we get

$$
A=(a t+b) \text {, }
$$

where $a=\frac{n}{\sqrt{n-1}}, b=n C_{2}$ and $n>1$.

Using equation (4.17), equation (4.10) leads to

$$
B=(a t+b)^{\frac{1}{n}} .
$$

Substituting equations (4.17) \& (4.18) in equation (4.8), we have

$$
\Lambda=\frac{3 a^{2}-2 n a^{2}+n^{2}}{n^{2}(a t+b)^{2}}-k
$$

where $4 \pi G f=k$.

Using equations (4.17), (4.18) and (4.19) in equation (4.7), we get

$$
8 \pi G \rho=\frac{-2 a^{2}+4 n a^{2}-2 n^{2}}{n^{2}(a t+b)^{2}}+2 k .
$$

as $\frac{1}{a^{2}}=\frac{1}{n}-\frac{1}{n^{2}}$.

Thus using equations (4.17) \& (4.18) in metric (4.1), the cosmological model is given by

$$
d s^{2}=d t^{2}-(a t+b)^{2} d x^{2}-(a t+b)^{\frac{2}{n}}\left(e^{-2 x} d y^{2}+e^{2 x} d z^{2}\right)
$$

Using equations (4.17), (4.18), (4.19) and (4.20) in equation (4.6), we have 
$\frac{d}{d t} \dot{C}^{2}+\left[2\left(1+\frac{2}{n}\right)\left(\frac{a}{a t+b}\right)\right] \dot{C}^{2}=\left[2\left(1+\frac{2}{n}\right)\left(\frac{a}{a t+b}\right)\right]$.

On integration of equation (4.22), we get

$$
\dot{C}=1 \text {, }
$$

which leads to

$$
C=t+b_{2}
$$

where $b_{2}$ is a constant of integration.

The value $\dot{C}=1$, So obtained agrees with the value used in the source equation. Thus creation field $\mathrm{C}$ is proportional to time $t$.

The physical parameters such as the spatial volume $(V)$, the mean anisotropic parameter $(\Delta)$ and the deceleration parameter $(q)$ are defined as

$$
\begin{gathered}
V=(a t+b)^{1+\frac{2}{n}}, \\
\Delta=2\left(\frac{n-1}{n+2}\right)^{2}, \\
q=2 \frac{(n-1)}{(n+2)}, \text { where } n>1 .
\end{gathered}
$$

Here $q>0$, for $n>1$.

\section{Plane Symmetric Universe}

We consider the plane symmetric metric in the form of

$$
d s^{2}=d t^{2}-A^{2}\left(d x^{2}+d y^{2}\right)-B^{2} d z^{2},
$$

where metric potentials $A, B$ are functions of time $t$ only and $\sqrt{-g}=A^{2} B$

It is assumed that creation field $C$ is a function of time $t$ only i.e. $C(x, t)=C(t)$ and

$$
{ }^{m} T_{j}^{i}=\operatorname{diag}(\rho,-p,-p,-p)
$$

The Hoyle-Narlikar field equations (2.1), with the help of equations (2.2) and (2.3) for the metric (5.1) given by

$$
\begin{gathered}
\frac{\dot{A}^{2}}{A^{2}}+2 \frac{\dot{A} \dot{B}}{A B}=8 \pi g\left(\rho-\frac{1}{2} f \dot{C}^{2}\right)+\Lambda, \\
\frac{\ddot{A}}{A}+\frac{\ddot{B}}{B}+\frac{\dot{A} \dot{B}}{A B}=8 \pi G\left(-p+\frac{1}{2} f \dot{C}^{2}\right)+\Lambda, \\
2 \frac{\ddot{A}}{A}+\frac{\dot{A}^{2}}{A^{2}}=8 \pi G\left(-p+\frac{1}{2} f \dot{C}^{2}\right)+\Lambda,
\end{gathered}
$$

where overhead $\operatorname{dot}\left({ }^{\prime}\right)$ denotes differentiation with respect to time $t$.

The conservation equation (2.7) leads to

$$
\frac{d}{d t} \dot{C}^{2}+2\left(2 \frac{\dot{A}}{A}+\frac{\dot{B}}{B}\right) \dot{C}^{2}=\frac{2 \dot{\rho}}{f}+\frac{2 \rho}{f}\left(2 \frac{\dot{A}}{A}+\frac{\dot{B}}{B}\right)+\frac{\dot{\Lambda}}{4 \pi G f} \text {, }
$$

where $p$ being isotropic pressure.

Following Hoyle and Narlikar, we have taken $p=0$, for the dust distribution. The source equation of $C$-field $C_{; i}^{j}=\frac{n}{f}$ leads to $C=t$ for large r. Thus $\dot{C}=1$. Using $p=0 \& \dot{C}=1$, equations (5.3)-(5.5) lead to

$$
\begin{aligned}
& \frac{\dot{A}^{2}}{A^{2}}+2 \frac{\dot{A} \dot{B}}{A B}=8 \pi G \rho-4 \pi G f+\Lambda, \\
& \frac{\ddot{A}}{A}+\frac{\ddot{B}}{B}+\frac{\dot{A} \dot{B}}{A B}=4 \pi G f+\Lambda \\
& 2 \frac{\ddot{A}}{A}+\frac{\dot{A}}{A^{2}}=4 \pi G f+\Lambda
\end{aligned}
$$

The field equations (5.7)-(5.9) is a system of three equations with four unknown parameters $A, B, \rho$ and $\Lambda$. To find the deterministic solution of the field equations, we need one extra condition. We assume that the shear scalar $(\sigma)$ is proportional to expansion scalar $(\theta)$ which leads to (Collins et al. [67])

$$
B=A^{n} \text {, }
$$

where $n$ is a arbitrary constant.

From equations (5.8) and (5.9), we get

$$
\frac{\ddot{B}}{B}-\frac{\ddot{A}}{A}+\frac{\dot{A} \dot{B}}{A B}-\frac{\dot{A}^{2}}{A^{2}}=0 .
$$

Using equations (5.10) and (5.11), we have

$$
2 \ddot{A}+2(n+1) \frac{\dot{A}^{2}}{A}=0 .
$$

To get the deterministic value of $A$, Let $\dot{A}=F(A)$,

which implies $\ddot{A}=F F^{\prime}$, where $F^{\prime}=\frac{d F}{d A}$. reduces to

With the help of equation (5.13), equation (5.12)

$$
\frac{d F^{2}}{d A}+\frac{2(n+1)}{A} F^{2}=0
$$

Integrating equation (5.14), we get 


$$
F^{2}=\frac{k_{1}^{2}}{A^{2(n+1)}}=\left(\frac{d A}{d t}\right)^{2}
$$

where $k_{1}^{2}$ is the constant of integration.

Using $\dot{A}=F(A)$ and solving equation (5.15), we get

$$
A=\left[(n+2)\left(k_{1} t+k_{2}\right)\right] \frac{1}{n+2},
$$

where $k_{2}$ is constant of integration.

Simplifying equation (5.16), we get

$$
A=(a t+b)^{\frac{1}{n+2}} \text {, }
$$

where $a=(n+2) k_{1}, \quad b=(n+2) k_{2}$ and $n>0$ \& $n \neq 1$.

Using equation (5.17), equation (5.10) leads to

$$
B=(a t+b)^{\frac{n}{n+2}} .
$$

Substituting equations (5.17) \& (5.18) in equation (5.9), we have

$$
\Lambda=-\frac{(2 n+1) a^{2}}{(n+2)^{2}(a t+b)^{2}}-k,
$$

where $4 \pi G f=k$.

Using equations (5.17), (5.18) and (5.19) in equation (5.7), we get

$$
8 \pi G \rho=\frac{(2 n+1)\left(2 a^{2}\right)}{(n+2)^{2}(a t+b)^{2}}+2 k .
$$

Thus, using equations (5.17) \& (5.18) in metric (5.1), the cosmological model is given by

$$
d s^{2}=d t^{2}-(a t+b)^{\frac{2}{n+2}}\left(d x^{2}+d y^{2}\right)-(a t+b)^{\frac{2 n}{n+2}} d z^{2} .
$$

Using equations (5.17), (5.18), (5.19) and (5.20) in equation (5.6), we have

$$
\frac{d}{d t} \dot{C}^{2}+\left[2\left(\frac{a}{a t+b}\right)\right] \dot{C}^{2}=\left[2\left(\frac{a}{a t+b}\right)\right] .
$$

On integration, equation (5.22) reduces to

$$
\dot{C}=1
$$

which leads to

$$
C=t+b_{3},
$$

where $b_{3}$ is a constant of integration.

The value $\dot{C}=1$, So obtained agrees with the value used in the source equation. Thus creation field $C$ is proportional to time $t$.
The physical parameters such as the spatial volume $(V)$, the mean anisotropic parameter $(\Delta)$ and the deceleration parameter $(q)$ are defined as

$$
V=(a t+b),
$$

$\Delta=2\left(\frac{n-1}{n+2}\right)^{2}=$ Constant, where $n>0 \quad \& \quad n \neq 1$ (5.26)

$$
q=2 .
$$

\section{Kantoski-Sachs Universe}

We consider the Kantowski-Sachs metric in the form of

$$
d s^{2}=d t^{2}-A^{2} d r^{2}-B^{2}\left(d \theta^{2}+\sin ^{2} \theta d \phi^{2}\right),
$$

where metric potentials $A, B$ are functions of time $t$ only and $\sqrt{-g}=A B^{2} \sin \theta$.

It is assumed that creation field $C$ is a function of time ${ }^{t}$ only

i.e. $C(r, t)=C(t)$ and ${ }^{m} T_{j}^{i}=\operatorname{diag}(\rho,-p,-p,-p)$

The Hoyle-Narlikar field equations (2.1) with the help of equations (2.2) and (2.3) for the metric (6.1) given by

$$
\begin{aligned}
& \frac{\dot{B}^{2}}{B^{2}}+2 \frac{\dot{A} \dot{B}}{A B}+\frac{1}{B^{2}}=8 \pi G\left(\rho-\frac{1}{2} f \dot{C}^{2}\right)+\Lambda, \\
& 2 \frac{\ddot{B}}{B}+\frac{\dot{B}^{2}}{B^{2}}+\frac{1}{B^{2}}=8 \pi G\left(-p+\frac{1}{2} f \dot{C}^{2}\right)+\Lambda, \\
& \frac{\ddot{A}}{A}+\frac{\ddot{B}}{B}+\frac{\dot{A} \dot{B}}{A B}=8 \pi G\left(-p+\frac{1}{2} f \dot{C}^{2}\right)+\Lambda,
\end{aligned}
$$

where overhead dot (') denotes differentiation with respect to time $t$.

The conservation equation (2.7) leads to

$\frac{d}{d t} \dot{C}^{2}+2\left(\frac{\dot{A}}{A}+2 \frac{\dot{B}}{B}\right) \dot{C}^{2}=\frac{2 \dot{\rho}}{f}+\frac{2 \rho}{f}\left(\frac{\dot{A}}{A}+2 \frac{\dot{B}}{B}\right)+\frac{\dot{\Lambda}}{4 \pi G f}$ (6.6)

where $p$ being iotropic pressure.

Following Hoyle and Narlikar, we have taken $p=0$, for the dust distribution. The source equation of $C$-field $C_{; i}^{j}=\frac{n}{f}$ leads to $C=t$ for large r. Thus $\dot{C}=1$. Using $p=0 \& \dot{C}=1$, equations (6.3)-(6.5) lead to

$$
\frac{\dot{B}^{2}}{B^{2}}+2 \frac{\dot{A} \dot{B}}{A B}+\frac{1}{B^{2}}=8 \pi G \rho-4 \pi G f+\Lambda,
$$




$$
\begin{aligned}
& 2 \frac{\ddot{B}}{B}+\frac{\dot{B}^{2}}{B^{2}}+\frac{1}{B^{2}}=4 \pi G f+\Lambda, \\
& \frac{\ddot{A}}{A}+\frac{\ddot{B}}{B}+\frac{\dot{A} \dot{B}}{A B}=4 \pi G f+\Lambda .
\end{aligned}
$$

The field equations (6.7)-(6.9) is a system of three equations with four unknown parameters $A, B, \rho$ and $\Lambda$. To find the deterministic solution of the field equations, we need one extra condition. We assume that the shear scalar $(\sigma)$ is proportional to expansion scalar $(\theta)$ which leads to (Collins et al. [67])

$$
A=B^{n}
$$

where $n$ is arbitrary constant.

From equations (6.8) and (6.9), we get

$$
\frac{\ddot{A}}{A}-\frac{\ddot{B}}{B}+\frac{\dot{A} \dot{B}}{A B}-\frac{\dot{B}^{2}}{B^{2}}-\frac{1}{B^{2}}=0
$$

Using equations (6.10) and (6.11), we have

$$
2 \ddot{B}+2(n+1) \frac{\dot{B}^{2}}{B}=\frac{2}{(n-1) B} .
$$

To get the deterministic value of $B$, Let $\dot{B}=F(B)$,

which implies $\ddot{B}=F F^{\prime}$, where $F^{\prime}=\frac{d F}{d B}$. reduces to

With the help of equation (6.13), equation (6.12)

$$
\frac{d F^{2}}{d B}+\frac{2(n+1)}{B} F^{2}=\frac{2}{(n-1) B} .
$$

Integrating equation (6.14), we get

$$
F^{2}=\frac{1}{n^{2}-1}=\left(\frac{d B}{d t}\right)^{2} .
$$

The constant of integration has taken to be zero for simplicity.

Using $\dot{B}=F(B)$ and solving equation (6.15), we get

$$
B=\frac{1}{\sqrt{n^{2}-1}} t+b
$$

where $b$ is a constant of integration.

Simplifying equation (6.16), we get

$$
B=(a t+b) \text {, }
$$

Where $a=\frac{1}{\sqrt{n^{2}-1}}$ and $n>1$.

Using equation (6.17), equation (6.10) leads to

$$
A=(a t+b)^{n} .
$$

Substituting equations (6.17) \& (6.18) in equation (6.8), we have

$$
\Lambda=\frac{a^{2}+1}{(a t+b)^{2}}-k
$$

where $4 \pi G f=k$.

Using equations (6.17), (6.18) and (6.19) in equation (6.7), we get

$$
8 \pi G \rho=\frac{2 n a^{2}}{(a t+b)^{2}}+2 k
$$

as $\frac{1}{a^{2}}=n^{2}-1$.

Thus using equations (6.17) \& (6.18) in metric (6.1), the cosmological model is given by

$$
d s^{2}=d t^{2}-(a t+b)^{2 n} d r^{2}-(a t+b)^{2}\left(d \theta^{2}+\sin ^{2} \theta d \phi^{2}\right) .
$$

Using equations (6.17), (6.18), (6.19) and (6.20) in equation (6.6), we have

$$
\frac{d}{d t} \dot{C}^{2}+\left[2(2+n)\left(\frac{a}{a t+b}\right)\right] \dot{C}^{2}=\left[2(2+n)\left(\frac{a}{a t+b}\right)\right]
$$

On integration, equation (6.22) reduces to

$$
\dot{C}=1 \text {, }
$$

which leads to

$$
C=t+b_{4}
$$

where $b_{4}$ is a constant of integration.

The value $\dot{C}=1$, So obtained agrees with the value used in the source equation. Thus creation field $\mathrm{C}$ is proportional to time $t$.

The physical parameters such as the spatial volume $(V)$, the mean anisotropic parameter $(\Delta)$ and the deceleration parameter $(q)$ are defined as

$$
\begin{aligned}
& V=(a t+b)^{n+2}, \\
& \Delta=2\left(\frac{n-1}{n+2}\right)^{2},
\end{aligned}
$$

$q=-\frac{(n-1)}{(n+2)}$, where $n>1$.

Here $q<0$, for $n>1$.

\section{Vil. Discussions}

\section{a) Spatial volume $(V)$}

In Fig. 1, the plots of spatial volume $(V)$ versus time $(t)$ are given for LRS B-II, LRS B-VI, PS \& K-S 
cosmological models which indicates that the models start evolving with finite volume at $t=0$ and expand infinitely with the increase in cosmic time $t$.

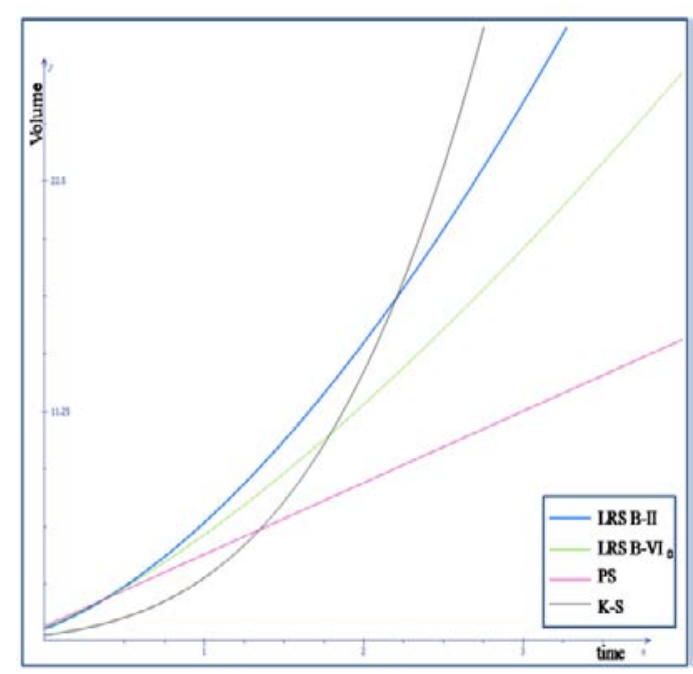

Figure 1: The plots of spatial volume $(V)$ verses time $(t)$.

\section{b) Energy density $(\rho)$}

In Fig. 2, the dynamics of energy density $(\rho)$ for cosmological models LRS B-II, LRS B-VI, PS \& K-S models are given. We observed that all models start with big-bang having infinite density and are decreasing functions of time which tends to positive finite value for large values of time $(t)$ representing steady state.

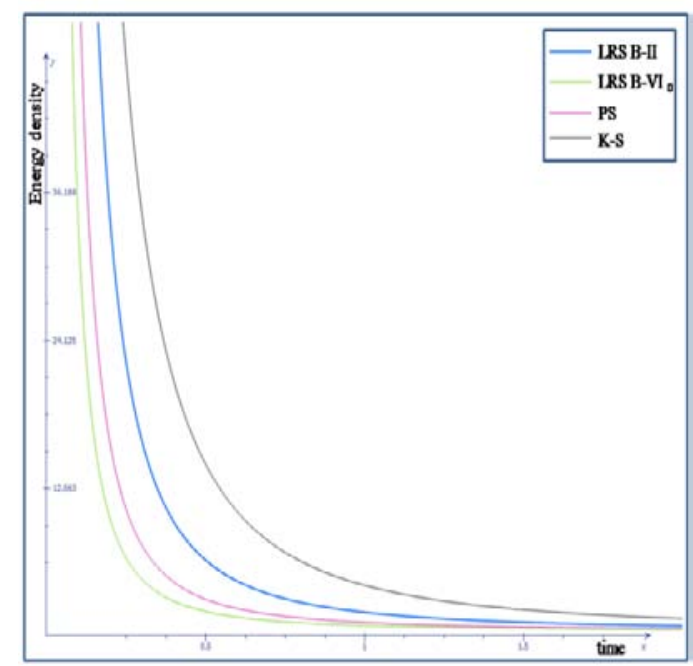

Figure 2 : The plots of energy density $(\rho)$ verses time $(t)$.

\section{c) Cosmological constant $(\Lambda)$}

In Fig. 3, the plots of cosmological constants $(\Lambda)$ versus cosmic time $(t)$ for LRS B-II, LRS B-VI, PS $\& \mathrm{~K}-\mathrm{S}$ cosmological models are given. We observed that the value of cosmological constant $(\Lambda)$ is initially infinite for all models. For K-S model, it is decreasing function of time and approaches to small positive value almost closer to zero at late times which matches with the recent $\mathrm{CMBR}$ observations.

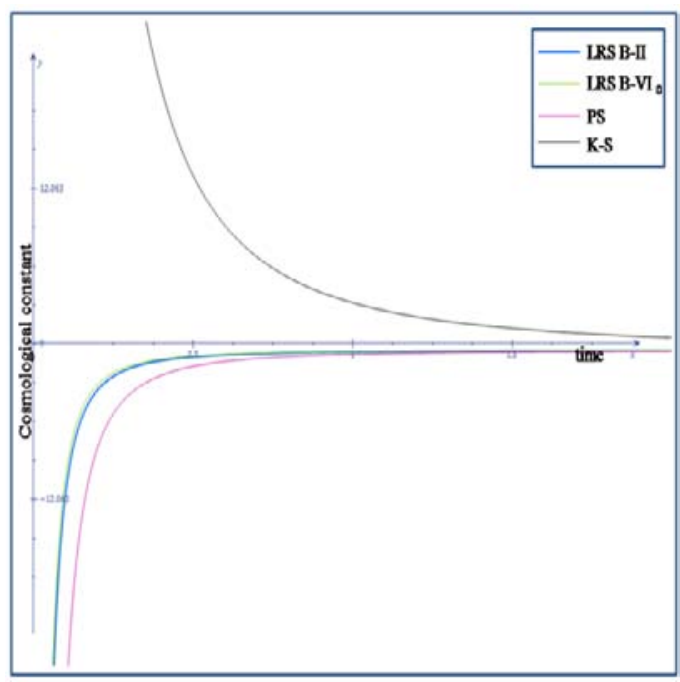

Figure 3 : The plots of cosmological constant $(\Lambda)$

$$
\text { verses time }(t)
$$

For LRS B-II, LRS B-VI $\mathrm{I}_{0}$ \& PS models, Cosmological constant $(\Lambda)$ is negative at initial stage but increases very rapidly with time and approaches to small negative value almost closer to zero at late times. We observed that LRS B-II, LRS B-VI $\&$ PS models with negative cosmological constant follow the study by authors' viz. Monerat et al. [68], Pedram et al. [69], Pradhan et al. [70] and Yadav [71] on cosmological models with negative cosmological constant.

\section{d) Deceleration parameter $(q)$}

For LRS B-II, LRS B-VI \& PS models, the value of deceleration parameter $q>0$ indicating that the models are decelerating and for $\mathrm{K}$-S model, the value of deceleration parameter $q<0$ indicating that the model is accelerating throughout the evolution of the universe.

\section{e) Mean Anisotropy Parameter $(\Delta)$}

It is also observed that for all cosmological models the mean anisotropy parameter $(\Delta)$ is constant. Hence the models are anisotropic throughout the evolution of the universe except at $n=1$ (i.e. the model does not approach isotropy).

\section{Vili. Conclusion}

LRS Bianchi type-II, LRS Bianchi type-VI, Plane symmetric and Kantowsaki-Sachs cosmological models have been investigated in Hoyle-Narlikar's creation field theory of gravitation. The source for energy momentum tensor is dust filled universe in the presence of time 
dependent cosmological constant $\Lambda(t)$. We conclude that all the models are expanding and anisotropic but LRS B-II, LRS B- $\mathrm{VI}_{0}$ \& PS models are decelerating while $\mathrm{K}-\mathrm{S}$ model is accelerating throughout the evolution of the universe. The creation field $C$ is directly proportional to time $t$. Hence the creation of matter increases as time increases which follows the results as obtained by Hoyle and Narlikar.

\section{References Références Referencias}

1. Smoot, G. F. et al., Astrophys. J., 396(1), L1,(1992).

2. Penrose, R., Phys. Rev. Lett., 14, 57,(1965).

3. Hawking, S. W., Phys. Rev. Lett., 15, 689,(1965).

4. Bondi, H., Gold, T., Mon. Not. Roy. Astro. Soc., 108, 252,(1948).

10 5. Hoyle, F., Narlikar, J. V., Proc. Roy. Soc. of Lon., Ser. A, Math. Phys. Sci., 278(1375), 465,(1964 a).

6. Hoyle, F., Narlikar, J. V., Proc. Roy. Soc. of Lon., Ser. A, Math. Phys. Sci., 282(1389), 178,(1964 b).

7. Hoyle, F., Narlikar, J. V., Proc. Roy. Soc. of Lon., Ser. A, Math. Phys. Sci., 282(1389), 191,(1964 c).

8. Narlikar, J. V., Nat. Phys. Sci., 242, 135,(1973).

9. Narlikar, J. V., Padmanabhan, T., The Ame. Phys. Soc., Phys. Rev. D, 32(8), 1928, (1985).

10. Narlikar et al., Astrophys. J., 585, 1,(2003).

11. Chatterjee, S., Banerjee, A., Gen. Rel. Grav., 36, 2, 303,(2004).

12. Singh, T., Chaubey, R., Astrophys. Space Sci., 321, 5,(2009).

13. Adhav, K. S., Katore, S. D, Bansod, A. S., Gadodia, P. S., Bulg. J. Phys., 37, 90,(2010).

14. Adhav, K. S., Gadodia, P. S., Bansod, A. S., Int. J. Theor. Phys., 50(9), 2720,(2011).

15. Katore, S. D., The Afr. Rev. Phys., 8:0024, 157, (2013).

16. Patil, V. R., Bolke, P. A., Bayaskar, N. S., Int. J. Theor. Phys., DOI: 10.1007/s10773-014-21759,(2014).

17. Perlmutter, S., et al., Astrophys J., 483(2), 565,(1997).

18. Perlmutter, S., et al., Nature, 391, 51,(1998).(astro$\mathrm{ph} / 9712212)$.

19. Perlmutter, S. et al., Astrophys. J., 517(2), 565,(1999)

20. Garnavich, P. M. et al., Astrophys. J. Lett., 493(2), L53,(1998 a).

21. Garnavich, P. M. et al., Astrophys. J., 509(1), 74, (1998 b).

22. Schmidt, B. P., et al., Astrophysical Jour., 507(1), 46, (1998).(astro-ph/9805200).

23. Riess, R.G. et al., The Astronomical J., 116(3), 1009, (1998).

24. Riess, R.G. et al., Pub. Astronomical Soc. Pacific (PASP), 112(776), 1284,(2000).
25. Riess, R. G. et al., Astrophysics J., 607(2), $665,(2004)$.

26. Zel'dovich, Ya. B., Sov. Phys. Usp., 11(3), 381,(1968).

27. Bergman, P. G., Int. J. Theo. Phys., 1(1), 25,(1968). Doi:10.1007/BF00668828.

28. Linde, A. D., JETP Lett., 19, 183,(1974).

29. Dolgov, A, D., (eds. Gibbons, G. W., Hawking, S. W. and Siklos, S. T. C.), Cambridge University Press, Cambridge, 449,(1983).

30. Dolgov, A. D., Sazhin, M. V., Zeldovich, Ya, B., Editions Frontieres,(1990).

31. Pavon, D., Physical Rev. D., 43, 375,(1991).

32. Weinberg, S., Rev. Mod. Phys., 61(1), 1,(1989).

33. Abers, E. S., Lee, B. W., Phys. Rep., 9(1), 1,(1973).

34. Langacker, P., Phys. Rep., 72, 185,(1981).

35. Bertolami, O., IL Nuovo Cimento B, Series 11, 93(1), 36,(1986).

36. Berman, M. S., Som, M. M., Int. J. Theor. Phys., 29, 1411,(1990).

37. Chen, W., Wu, Y. S., Phys. Rev. D, 41(2), 695,(1990).

38. Carvalho, J. C., Lima, J. A. S., Waga, I., Phys. Rev. D, 46(6), 2404,(1992).

39. Overduin, J. M., Astrophys. J., 517(1), L1, (1999).

40. Olson, T. S., Jordan, T. F., Phys. Rev. D., 35(10), 3258,(1987).

41. Gasperini, M., Class. Quant. Grav., 5, 521,(1988).

42. Pebbles, P. J. E., Ratra, B., Astrophys. J. Lett., 325, L17,(1988).

43. Abdel-Rahman, A. M. M., Phys. Rev. D, 45, 3497,(1992).

44. Maia, M. D., Silva, G. S., Phys. Rev. D, 50, 7233,(1994)

45. Silveira, V., Waga, I., Phys. Rev. D., 50, 4890,(1994).

46. Moffat, J. W., Phys. Lett. B, 357, 526,(1995).

47. Torres, L. F. B., Waga, I., Mon. Not. R. Astron. Soc., 279, 712,(1996).

48. Abdussattar, Vishwakarma, R. G., Pramana - J. Phys., 47, 41,(1996)

49. Hoyle, F., Burbidge, G., Narlikar, J. V., Mon. Not. R. Astron. Soc., 286, 173,(1997).

50. Podariu, S., Ratra, B., Astrophys. J., 532 , 109,(2000).

51. Carmeli, M., Kuzmenko, T., Int. J. Theor. Phys., 41(1), 131,(2002).

52. Liu, H., Wesson, P. S., The Astrophysical J., 562, 1 , (2001).

53. Cunha, J. V., Santos, R. C., Int. J. Mod. Phys. D, 13, 1321,(2004).

54. Carneiro, S., Lima, J. A., Int. J. Mod. Phys. A, 20, 2465,(2005).

55. Pradhan, A., Yadav, A. K., Yadav, L., Czech. J. Phys., 55(4), 503,(2005).

56. Singh C. P., Suresh Kumar, Int. J. Theor. Phys., 47(12), 3171,(2008). 
57. Katore, S. D., Rane, R. S., Wankhede, K. S., Int. J. Theo. Phys., 49(9), 2105,(2010).

58. Tiwari, R. K., Sharma, S., Chin. Phys. Lett., 28(2), 020401-(1-3), (2011).

59. Dwivedi, U. K., Int. J. of Adv. Res. in Eng. \& App. Sci. (IJAREAS), 1(1), 31,(2012).

60. Amirhashchi, H., Mohamadian, H., Pramana- J. of Physics, 78(4), 651,(2012).

61. Bali, R., Saraf, S., Proc. Natl. Acad. Sci., India, Sec. A, Phys. Sci., 83(1), 29,(2013 a).

62. Bali, R., Saraf, S., Prespacetime J.,4(5),545,(2013 b).

63. Rahman, M. A., Ansari, M., Prespacetime J., 4(9), 871,(2013).

64. Ghate, H. R., Salve, S. A., Res. J. Recent Sci, 3(ISC2013), 53,(2014 a).

65. Ghate, H. R., Salve, S. A., Prespacetime J., 5(3), 198,(2014 b).

66. Ghate, H. R., Salve, S. A., Int. J. Sci. \& Eng. Res., 5(6), 254,(2014 C).

67. Collins, C. B., Glass, E. N., Wilkinson, D. A., Gen. Rel. Grav., 12, 805,(1980).

68. Monerat, G. A., Correea Silva, E. V., Oliveira-Neto, G., Ferreira Filho, L. G., Lemos, N. A., Phy. Rev. D, 73(4), 044022,(2006). Doi: 10.1103/PhyRevD. 73.044022.

69. Pedram, P., Mirzaei, M., Jalalzadeh, S., Gousheh, S. S., Gen. Rel. Grav., 40, 1663, (2008).

70. Pradhan, A., Kumhar, S. S., Yadav, P., Jotania, K., Int. J. Math. Eng. \& Sci. (IJMES), 1(2), 74, (2012).

71. Yadav, A. K., Elec. J. Theor. Phys., 10, 169, (2013). arXiv:0911.0177[gr-qc]. 
This page is intentionally left blank 


\section{Explanation of Dark Matter and Dark Energy Phenomena By Alexander A. Antonov Research Centre of Information Technologies "TELAN Electronics", Ukraine} Abstract- It is demonstrated that 'dark matter' and 'dark energy' in our universe are definitely registered physical attributes testifying to the existence of other parallel universes that form the existent Multiverse. This real Multiverse is referred to as hidden, because other universes are not observable for the inhabitants of the parallel universes it includes. The structure of the hidden Multiverse complies with two principles: the principle of the physical reality of imaginary and complex numbers proved by the author and the similarity principle suggested by the author. The latter implies that the laws of nature governing all parallel universes are identical; however, certain differences are still possible. For instance, time flows in different directions with regard to the time in our universe.

Keywords: dark matter, dark energy, multiverse, parallel universe, antiverse, imaginary numbers, special theory of relativity.

GJSFR-A Classification : FOR Code: 020199

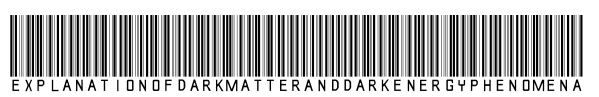

Strictly as per the compliance and regulations of:

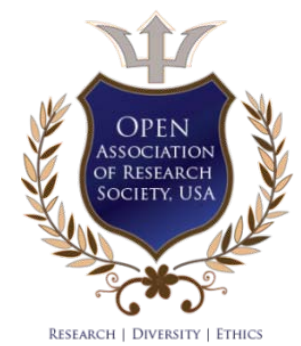

(C) 2015. Alexander A. Antonov. This is a research/review paper, distributed under the terms of the Creative Commons AttributionNoncommercial 3.0 Unported License http://creativecommons.org/licenses/by-nc/3.0/), permitting all non commercial use, distribution, and reproduction in any medium, provided the original work is properly cited. 


\title{
Explanation of Dark Matter and Dark Energy Phenomena
}

\author{
Alexander A. Antonov
}

Abstract- It is demonstrated that 'dark matter' and 'dark energy' in our universe are definitely registered physical attributes testifying to the existence of other parallel universes that form the existent Multiverse. This real Multiverse is referred to as hidden, because other universes are not observable for the inhabitants of the parallel universes it includes. The structure of the hidden Multiverse complies with two principles: the principle of the physical reality of imaginary and complex numbers proved by the author and the similarity principle suggested by the author. The latter implies that the laws of nature governing all parallel universes are identical; however, certain differences are still possible. For instance, time flows in different directions with regard to the time in our universe.

Keywords: dark matter, dark energy, multiverse, parallel universe, antiverse, imaginary numbers, special theory of relativity.

\section{InTRODUCTION}

n order to explicate the statement that dark matter and dark energy testify to the existence of other universes, in addition to ours, that form the existent Multiverse, let us start with explaining what kind of Multiverse is in question.

The Multiverseis understood as any hypothetical set of all parallel universes. The universes are referred to as parallel because, despite their boundlessness, they never intersect. Numerous hypotheses about the Multiverse have been suggested by physicists, mathematicians, astronomers, engineers, philosophers and science fiction writers. Some of these hypotheses are outlined in [1] - [11], from which it can be seen that those suggested by scientists are even more extraordinary than the ones suggested by science fiction writers.

They are so extraordinary that, as stated in [12], all the hypotheses about other universes, except for the one discussed in this article, will never be verified experimentally. In other words, the Multiverse they describe is actually non-existent for us.

However, [13] - [17] suggest a different concept of the Multiverse. This concept is that the Multiverse actually exists and consists of a set of parallel universes that comply with the similarity principle. That is, the concept states that the laws of nature governing these parallel universes have much in common.

Author: Research Centre "Telan Electronics", Kiev, Ukraine. e-mail: telan@bk.ru
Therefore, other parallel universes are accessible for the inhabitants of these universes to visit. These visits can prove the reality of this Multiverse. However, many inhabitants of these universes, including the earthlings, believe these parallel universes to be non-existent, because by their physical nature they are transparent and, due to this, are not observable for the inhabitants of other parallel universes. Therefore, this Multiverse can be referred to as hidden.

\section{il. The Discovery and Investigation OF IMAGINARY NuMBERS}

The history of the Multiverse hypothesis discussed in this paper starts with the discovery of an imaginary unit $\boldsymbol{i}=\sqrt{-1}$ by Scipione del Ferro (1465 1525), Niccolò Fontana Tartaglia (1499 - 1557), Gerolamo Cardano (1501 - 1576), Lodovico Ferrari (1522 - 1565) and Rafael Bombelli (1526 - 1572) [18]. The discovery may have been made even earlier by Paolo Valmes (d.1486), who was sentenced to death at the stake by the Spanish inquisitor Tomás de Torquemada (1420 - 1498) [19].

Now, with the works of prominent mathematicians such as Abraham de Moivre (1667 1754), Leonhard Euler (1707 - 1783), Jean Le Rond D'Alembert (1717 - 1783), Caspar Wessel (1745 1818), Pierre-Simon de Laplace (1749 - 1827), JeanRobert Argand (1768 - 1822), Johann Carl Friedrich Gauss (1777 - 1856), Augustin Louis Cauchy (1789 1857), Karl Theodor Wilhelm Weierstrass (1815 - 1897), William Rowan Hamilton (1805 - 1865), Pierre Alphonse Laurent (1813 - 1854), Georg Friedrich Bernhard Riemann (1826 - 1866), Oliver Heaviside (1850 - 1925), Jan Mikusiński (1913 - 1987) and many others, a consistent theory of the functions of a complex variable has been developed. However, this theory does not explain the physical meaning of imaginary and complex numbers.

In 1826, Felix Savary $(1797$ - 1841) discovered the alternating electric current [20]. Charles Proteus Steinmetz (1865 - 1923) suggested using the symbolic method to describe it [21], thus introducing the notion of complex frequency into electrical circuit theory. However, textbooks on the electric circuit theory do not explain its meaning. 
Imaginary and complex numbers are now widely used in other exact sciences as well - in optics, hydraulics, mechanics, acoustics, and so on. However, these sciences do not explain the physical meaning of imaginary and complex numbers, either.

In the early twentieth century, Joseph Larmor (1857 - 1942), the Nobel Prize winner Hendrik Antoon Lorentz (1853 - 1928), Jules Henri Poincaré (1854 1912), the Nobel Prize winner Albert Einstein (1879 1955) and other prominent scientists developed the special theory of relativity (STR). Its formulae describing relativistic effects at superluminal speeds used imaginary numbers. However, no one has been able to explain the physical meaning of those numbers, either at that time or at the present time. Therefore, one of the formulations of the second postulate of the STR states that imaginary numbers do not have any physical meaning [22]

However, not all scientists found the current version of the STR sufficiently convincing. So far, several hundred scientific works criticizing different aspects of the STR have been published; among the latest, [23] [26] can be cited.

In the twenty-first century, the MINOS and OPERA experiments were performed; they attempted to refute the fundamental principle of the STR - the unbreakable barrier of the speed of light - and, thus, to prove the physical reality of imaginary numbers. However, the physics community believed these experiments to be insufficiently reliable.

\section{iil. The Principle of the Physical Reality of IMAGINARY AND COMPLEX Numbers INTERPRETATION}

Nevertheless, [27] - [32] present the results of other research that proves the physical reality of imaginary and complex numbers, both theoretically and experimentally. The evidence was obtained by investigating oscillation processes in linear electrical circuits, which allows the validity of the experiments to be verified in any radio electronics laboratory. Therefore, the results of these experiments are presumably reliable.

These publications also demonstrate that the physical reality of imaginary and complex numbers explains the existence of shock oscillations in nature. Otherwise, tsunami, percussion instruments and many other natural phenomena would not exist.

However, nature is consistent. Therefore, science that tries to understand and explain the laws of nature must be integral and consistent. Separate scientific disciplines - the special and general theory of relativity, quantum mechanics, mathematics, radio electronics, optics, and so on - that exist only because of people's limited intellectual potential, must harmonize their theories and hypotheses.
The understanding and use of mathematics in all other sciences must be even more consistent. Consequently, the mathematical principle of the physical reality of imaginary and complex numbers, regardless of the branch of science to which it belongs, is universally true and applicable not only to electrical circuit theory [27] - [32], but also to the STR [33], quantum mechanics [34], [35], optics, and all other sciences.

\section{Adjustment of the Special Theory of ReLATIVITY}

Therefore, theories that have been developed on the basis of the above erroneous statement of the STR, which denies the physical reality of imaginary numbers, must now be revised and adjusted accordingly.

Let us demonstrate how this can be done, for example, in the STR.

To solve the problem, it is obviously necessary to explain the meaning of the relativistic formulae at superluminal speeds, and for that, it is enough to consider any one of them. For example, let us take the formula:

$$
\begin{gathered}
m=\frac{m_{0}}{\sqrt{1-(v / c)^{2}}} \\
\Delta t=\Delta t_{0} \sqrt{1-(v / c)^{2}}
\end{gathered}
$$

where $\boldsymbol{m}_{\boldsymbol{0}}$ is the rest mass of a moving body (e.g.,

of an elementary particle);

$\boldsymbol{m}$ is the relativistic mass of a moving body;

$\Delta t_{0}$ is the rest time of a moving body;

$\Delta \boldsymbol{t}$ is the relativistic time of a moving body;

$\boldsymbol{v}$ is the velocity of the body; and

$\boldsymbol{c}$ is the speed of light.

As can be seen, according to formula (1), at subluminal speeds at $\boldsymbol{v}<\boldsymbol{c}$ the mass of the elementary particles, referred to as tardyons (or bradyons), is measured with real numbers, and at superluminal speeds at $\boldsymbol{v}>\boldsymbol{c}$ the mass of the elementary particles, referred to as tachyons, is measured with imaginary numbers. At the same time, tachyons cannot be detected in our universe (let us call it the tardyon universe for convenience), because they are located elsewhere: this other place can be referred to as the tachyon universe. According to formula (2), time in the tardyon universe is measured with real numbers, and in the tachyon universe it is measured with imaginary numbers.

It is very likely that inhabitants of the tachyon universe (or inhabitants of other, more remote universes) have somehow been visiting the Earth. In order for 
inhabitants of different universes to visit the planet Earth, those other universes must comply with the 'similarity principle', i.e. the fundamental physical, chemical, biological, and other laws in them must be identical or have much in common.
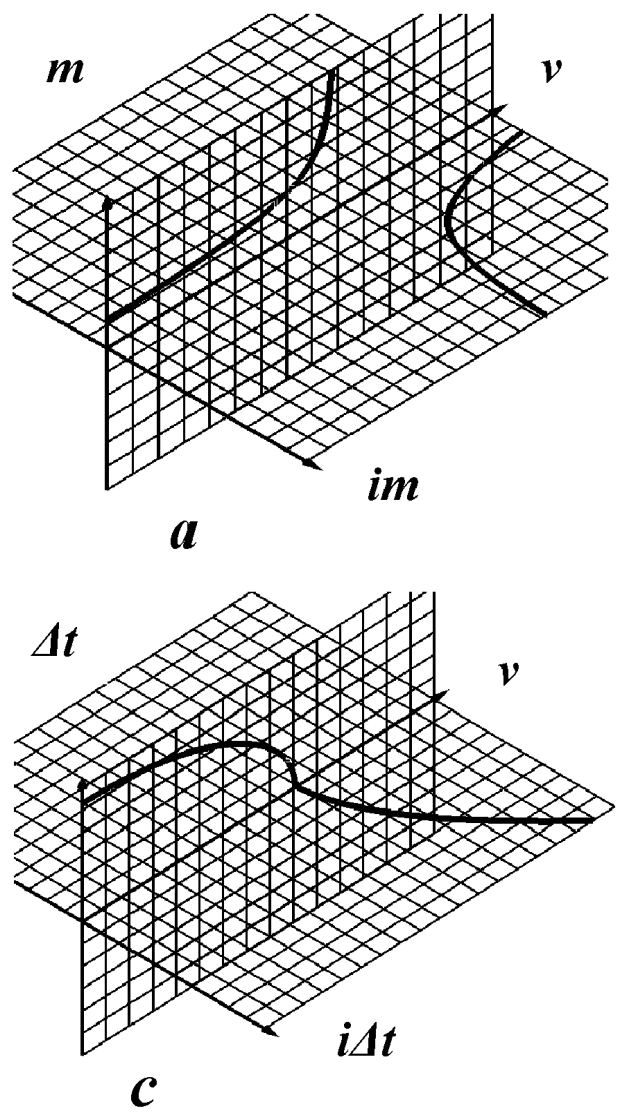

However, as can be seen (see Fig. 1a and 1c), the graphs of formulae (1) and (2) at $\boldsymbol{v}<\boldsymbol{c}$ and at $\boldsymbol{v}>\boldsymbol{c}$ look different, i.e. they do not comply with the similarity principle. Therefore, formulae (1) and (2) must be adjusted; this can be done as follows

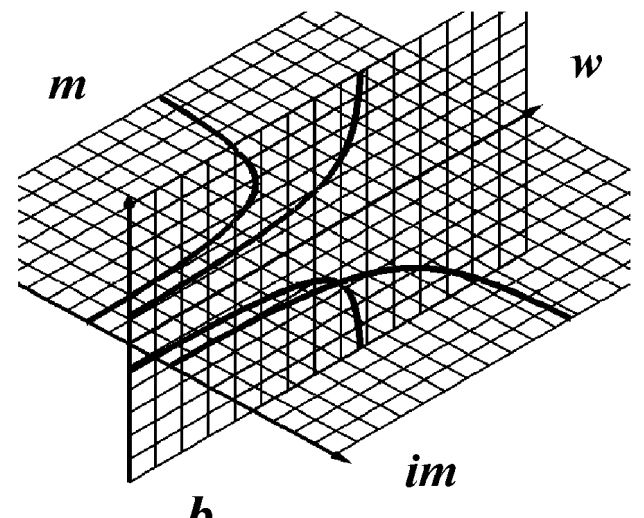

b

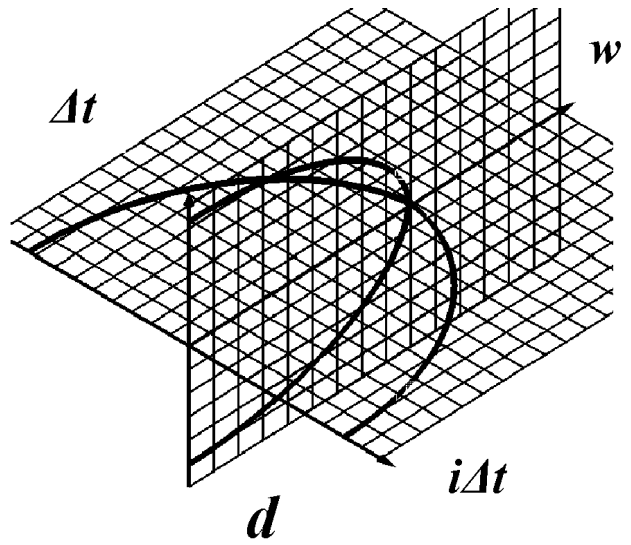

Figure 1: Graphs of functions (1) - (4)

$$
\begin{gathered}
m=\frac{(i)^{q} m_{0}}{\sqrt{1-(v / c-q)^{2}}}=\frac{(i)^{q} m_{0}}{\sqrt{1-(w / c)^{2}}} \\
\Delta t=(i)^{q} \Delta t_{0} \sqrt{1-(v / c-q)^{2}}= \\
=(i)^{q} \Delta t_{0} \sqrt{1-(w / c)^{2}}
\end{gathered}
$$

where $\boldsymbol{k}=\lfloor v / \boldsymbol{c}\rfloor$ is the discreet floor function of the argument $v / c$;

$\boldsymbol{w}=\boldsymbol{v}-\boldsymbol{q} \boldsymbol{c}$ is the local velocity, for each universe, which can take values only in the range $0 \leq c w<$;

$\boldsymbol{v}$ is the velocity measured from our tardyon universe, which, hence, can be referred to as the tardyon velocity.

Naturally, other relativistic formulae of the STR have the same shortcomings and can be adjusted in a similar way.

\section{Dark Matter and Dark Energy}

As can be seen (Fig. $1 \mathrm{~b}$ and $1 \mathrm{~d}$ ), in formulae (3) and (4) the value $\boldsymbol{q}=\mathbf{0}$ corresponds to the tardyon universe, and the value $\boldsymbol{q}=\mathbf{1}$ to the tachyon universe. It can be seen from Fig. $1 \mathrm{~b}$ and 1d that the Multiverse must have at least two more parallel universes that correspond to $\boldsymbol{q}=\mathbf{2}$ and $\boldsymbol{q}=\mathbf{3}$. It is logical to refer to them as the tardyon antiverse and the tachyon antiverse, because they correspond to the values of mass, time and other quantities determined with the relativistic formulae of the STR, measured with negative real numbers and negative imaginary numbers.

Let us note, at the same time, that in the Multiverse structure described in this article, the tardyon universe and the tardyon antiverse are never adjacent, but they are separated by the tachyon universe and the tachyon antiverse. Similarly, the tachyon universe and the tachyon antiverse are never adjacent, because they are separated by the tardyon universe and the tardyon 
antiverse. Thus, annihilation of the corresponding universes and antiverses is prevented.

Further, the value $\boldsymbol{q}=\mathbf{4}$ in formulae (3) and (4) once again corresponds to the tardyon universe. However, this can be either our own universe or another tardyon universe. The form of the structure of the Multiverse depends on this, as well as on other yet unknown circumstances. Furher research will reveal what this form actually is.

In the case the values $\boldsymbol{q}=\mathbf{0}$ and $\boldsymbol{q}=\mathbf{4}$ correspond to the same universe - our tardyon universe, the Multiverse has a ringed structure. It includes one tardyon, one tachyon, one anti-tardyon and one antitachyon universe. They adjoin each other in this particular order. Other parallel universes are not seen from any of the universes.

In the case the values $\boldsymbol{q}=\mathbf{0}$ and $\boldsymbol{q}=\mathbf{4}$ correspond to different tardyon universes, the Multiverse has a helical stricture. Then, it includes several tardyon, tachyon, anti-tardyon and anti-tachyon universes, once again, in this particular sequence. However, the total number of universes that form the helical Multiverse is likely to be limited and, eventually, a subsequent tardyon universe would coincide with our tardyon universe. This number must be a multiple of four. It can be estimated by comparing the mass of our universe and the total mass of dark matter and dark energy of other parallel universes. With this in mind, we can conclude that this number is, most likely, twenty-four. Then the structure of the Multiverse would take the form of a screw collar.

In the hidden Multiverse discussed in this publication, other parallel universes can not be observed from any of the parallel universes, although, certain indirect evidence of their existence can be registered. For example, this is dark matter and dark energy, where no traces of any known chemical elements or other signs of real matter can be found exactly for the reason that they are not in our universe. However, they can be detected, if we penetrate into these other universes from our universe. This can be done through portals.

Therefore, it is time to discuss portals. The matter is that, due to some unknown processes, the relative position of the universes forming the Multiverse is not locked. They float relative to each other in the extra spatial dimensions. Therefore, they sometimes touch, and even partially penetrate each other. Then, at points of these mutual penetrations, certain transition zones, or portals (these have nothing to do with the socalled 'wormholes' mentioned in other hypotheses of the Multiverse, because they operate in a different way), appear. These portals enable the inhabitants of adjacent parallel universes to visit each other. These portals, obviously, can be of different sizes. When they are small, we call them geopathic zones. When they are large (from hundreds meters to several kilometres and more), we refer to them as the anomalous zones.

In portals, the quantity $\boldsymbol{q}$ takes non-integral values and changes rapidly from an integral value corresponding to one of the adjacent parallel universes to the integral value corresponding to another adjacent parallel universe. It is not clear how people who get into portals would perceive these changes. If a human body can handle them, then people would be able to visit other parallel universes through portals.

It is noteworthy that all trans-portal areas of the adjacent (and, most likely, of all) parallel universes on the planet Earth are accessible for people to visit and are even habitable. Therefore, all parallel universes are most likely inhabited. Otherwise, if the trans-portal area of at least one of the numerous portals on the planet Earth turned to be, for instance, in the empty interplanetary space, then, according to the law of connected vessels, the planet Earth would have no air. The same is true for all other unfavourable border situations.

Now it is possible to explain how both elementary particles and other physical objects (possibly, even living beings) can make transitions from one parallel universe to another. It turns out that in the Multiverse, portals are used for this purpose. Therefore, there is no need to break the speed of light barrier in accordance with the relativistic formulae of the STR. This is similar to the fact that you do not have to break through a wall to move from one room of your apartment to another, because you can use a door.

Finally, we do not rule out that the Multiverse is even more complicated, because not only complex numbers are physically real, but hypercomplex numbers, as well [36]. Then the structure of the Multiverse becomes even more complex, as well as the formulae describing relativistic effects.

\section{Vi. Conclusion}

Thus, the phenomenon of dark matter and dark energy is explained by the existence of the hidden Multiverse that has the structure described in this article, where parallel universes are not observable or anyhow registered from our universe. Other parallel universes cannot be seen from any other universe, either. Any parallel universe can be observed only from this same universe, certainly, on condition we get there.

Nevertheless, it turns out that the existence of these unobservable parallel universes is possible to detect based on some indirect physical indicators. These indicators have been named dark matter and dark energy, with the difference between the two being explained, possibly, by the fact that dark matter corresponds to the closer and dark energy - to the more remote parallel universes. 
Therefore, the phenomenon of dark matter and dark energy proves the existence of the hidden Multiverse described in this article. The reality of this Multiverse will be confirmed incontestably and finally by people visiting other parallel universes through portals and by exploration of these universes.

\section{References Références Referencias}

1. Lewis D. (1986). On the Plurality of Worlds. Basil Blackwell, Oxford.

2. Linde D. (1990). Particle Physics and Inflationary Cosmology. Harwood Academic Publishers, Switzerland.

3. Deutsch, D. (2002). The structure of the multiverse. Proceedings of the Royal Society A, 458, 2911 2923. (doi: 10.1098/rspa.2002.1015)

4. Tegmark, M. (2003). Parallel Universes, Scientific American. 288(5), 40 - 51.

5. Green B. (2004). The Elegant Universe: Superstrings, Hidden Dimensions, and the Quest for the Ultimate Theory, W. W. Norton \& Company. NY.

6. Ellis, G.F.R., Kirchner U. and Stoeger, W.R. (2004). Multiverses and physical cosmology, Monthly Notices of the Royal Astronomical Society. 347(3). 921 - 936. (doi: 10.1111/j.1365-2966.2004.07261.x).

7. Steinhardt P. J. and N. Turok, (2007). Endless Universe: Beyond the Big Bang, Doubleday. NY.

8. Tegmark, M. (2008). The mathematical universe. Foundation of Physics. 38(2). $101-150$.

9. Carr, B. ed., (2009). Universe or Multiverse? Cambridge Univ. Press. Cambridge.

10. Lucash V. N. and Mikheyeva, E.V. (2010). Physical cosmology, Physmathlit Publishing, Moscow.

11. Greene B, (2011). The Hidden Reality: Parallel Universes and the Deep Laws of the Cosmos, Knopf. NY.

12. Ellis G.F.R. (2011). Does the Multiverse Really Exist? Scientific American. 305, 38 - 43.

13. Antonov A.A. (2011). Structure of the Multiverse. British Journal of Science. 2(2), $51-60$.

14. Antonov A.A. (2012). Multiuniverse. Time Travels, International Journal of Pure and Applied Sciences and Technology, 12(2) $43-56$.

15. Antonov A. A. (2012). Earth. Portals. Parallel Universes. American Journal of Scientific and Industrial Research. 3(6): $464-473$.

16. Antonov A. A. (2012). Discovery of the Real Multiuniverse. Encyclopedia of Russian Thought: Reports to Russian Physical Society. 16(3). 3 - 20.

17. Antonov A. A. (2013). Cognition of the Multiverse as a factor facilitating the development of humanity. Russian Physical Though Journal. 1(12): $6-77$.

18. Weisstein E. W. (2005). The CRC Concise Enciclopedia of Mathematics. 3-rd ed. CRS Press. Roca Raton, FL .
19. Beckmann P. (1976). A History of $\pi$. 3 -rd edition. St. Martin's Press. NY.

20. Blanchard, Ju. (1941). The History of Electrical Resonance. Bell System Technical Journal. 20(4): $415-433$.

21. Steinmetz C. P., Berg E. J. (1900). Theory and calculation of alternating current phenomena. Electrical World and Engineer Inc., NY.

22. Antonov A. A. (2014). Verification of the second postulate of the special relativity theory. Global Journal of Science Frontier Research A: Physics and Space Science. 14(3). 51 - 59.

23. Goldsmith D. (1997). Einstein's Greatest Blunder?: The Cosmological Constant and Other Fudge Factors in the Physics of the Universe. Harvard University Press. Cambridge. MA.

24. Kalinin L. A. (2003). Einstein's cardinal errors. Editorial URSS Publishing. Moscow.

25. Artekha S. N. (2007). Criticism of the basics of the relativity theory. 2nd edition. LKI Publishing. Moscow.

26. Albert D. Z. and Galchen R. (Feb. 2009). Was Einstein Wrong? A Quantum Threat to Special Relativity. Scientific American.

27. Antonov A. A. and Buzhev V. M. (1974). Means of rising deflecting currents for spiral beam sweep on the CRT screen. Patent of USSR \# 433650.

28. Antonov A. A. (2008). Physical Reality of Resonance on Complex Frequencies. European Journal of Scientific Research. 21(4): 627 - 641.

29. Antonov A. A. (2009). Resonance on Real and Complex Frequencies. European Journal of Scientific Research. 28(2): 193 - 204.

30. Antonov A.A. (2010). Oscillation Processes as a Tool of Physics Cognition. American Journal of Scientific and Industrial Research. 1(2): 342 - 349.

31. Antonov A.A. (2010). Solution of Algebraic Quadratic Equations Taking into Account Transitional Processes in Oscillation Systems. General Mathematics Notes.1(2): $11-16$.

32. Antonov A. A. (2011). Resonant Processes as a Tool for Revealing the Universe's Hidden Dimensions. American Journal of Scientific and Industrial Research. 2(4): $567-572$.

33. Antonov A. A. (2014). Correction of the special theory of relativity: physical reality and nature of imaginary and complex numbers. American Journal of Scientific and Industrial Research. 5(2): 40- 52.

34. Spenkov, G. P. (2013). Physical meaning of imaginary unit i. Encyclopedia of Russian Thought: Reports to Russian Physical Society. 20(2), 70 - 81.

35. Ahkosov Ju. L. Florensky physical plane as the basis for the macrocosm substratum. Encyclopedia of Russian Thought: Reports to Russian Physical Society. 23(2). $46-82$.

.


36. Kantor I. L. and Solodovnikov A. S. (1989). Hypercomplex numbers. Berlin: Springer Verlag. 


\section{Possible Role of the Gailitis Resonance in Low Energy Nuclear Fusion Experiments}

By Chi-Yu Hu

California State University Long Beach, United States

Abstract- The physical mechanism for the formation of the Gailitis resonances has been established in a recent precision calculation. According to the condition described in the low energy nuclear fusion experiments, the likelihood of Gailitis resonance induced low energy nuclear fusion exists. In this note, the properties of Gailitis resonance, the compound nuclear resonances, the conservation laws of energy, parity and the nuclear angular momentum will be used to support the possibility of Gailitis resonance induced low energy nuclear fusion.

Keywords: resonances, low energy nuclear fusion.

GJSFR-A Classification : FOR Code: 020199

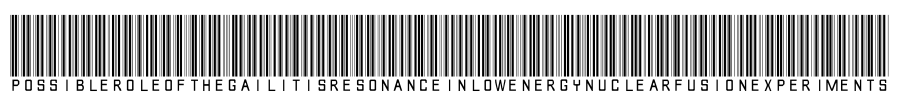

Strictly as per the compliance and regulations of:

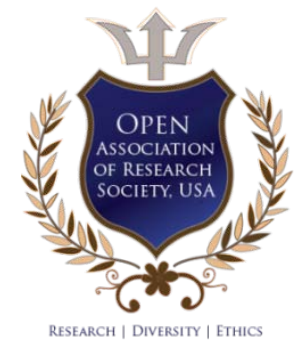

(c) 2015. Chi-Yu Hu. This is a research/review paper, distributed under the terms of the Creative Commons AttributionNoncommercial 3.0 Unported License http://creativecommons.org/licenses/by-nc/3.0/), permitting all non commercial use, distribution, and reproduction in any medium, provided the original work is properly cited. 


\section{Possible Role of the Gailitis Resonance in Low Energy Nuclear Fusion Experiments}

Chi-Yu Hu

\begin{abstract}
The physical mechanism for the formation of the Gailitis resonances has been established in a recent precision calculation. According to the condition described in the low energy nuclear fusion experiments, the likelihood of Gailitis resonance induced low energy nuclear fusion exists. In this note, the properties of Gailitis resonance, the compound nuclear resonances, the conservation laws of energy, parity and the nuclear angular momentum will be used to support the possibility of Gailitis resonance induced low energy nuclear fusion.
\end{abstract}

Keywords: resonances, low energy nuclear fusion.

\section{INTRODUCTION}

$\mathrm{R}$ esonances are universal phenomena. They are well known to appear in numerous mechanical or electrical systems, large or small. Here their effects are easily recognizable. Their origin in microscopic systems are much more subtle and diverse. There are only a few well known and well understood resonances in atomic and nuclear systems, such as Feshbach resonances, nuclear magnetic resonances, only recently, the Gailitis resonances. The six-body resonance in muoncatalyzed fusion, typically represented by:

$$
(t \mu)_{00}+\left(D_{2}\right)_{\nu_{i}, k_{i}} \longrightarrow\left[(d t \mu)_{11}, d e e\right]_{\nu_{f}, k_{f}},
$$

where $(t \mu)_{00}$ is muon atom in ground state, $\left(D_{2}\right)_{\nu_{i} k_{i}}$ is a deuterium molecule in rot-vibrational state represented by the quantum number $\nu_{i}, k_{i}, d$ is the nucleus of deuterium (deuteron), and $e$ represents the electron.

The right hand side of Eq. (1) is a giant complex molecule in rot-vibrational quantum states $\nu_{f}, k_{f}$ Eq. (1) represents a resonant process where the $(t \mu)_{00}+d$ sub-system on the left-hand side transfers $0: 66 \mathrm{eV}$ of energy to the rot-vibrational energy of the large complex molecule and becomes a bounded small molecular ion $(d t \mu)_{11}$ inside the large complex molecule in Eq. (1). Resonant property enables the transferring of energy between two systems differing in size by several orders of magnitudes.

The properties of Gailitis resonance have been revealed only recently [1]. Its lowest quantum states occasionally showed up in atomic calculations. They are called shape resonances. Because of their short-life time, they played little role in most physical processes.

According to [1], in a three-body colliding system the Gailitis resonances occur when the energy of the incoming colliding charged particle satisfies the resonant condition

$$
E_{m}=m\left|\mu_{l}\right| /\left\langle y^{2}\right\rangle_{m}, \quad m=1,2,3 \ldots
$$

All quantities are expressed in mass normalized Jacobi coordinate system, energy in atomic units: $m$ is the Gailitis resonance quantum number, $1 / y_{m}^{2}$ is the Coulomb field of the incoming charged particle at the center of mass of the target, $\mu_{l}$ is the the electric dipole moment of the target system. During the life time of the resonance, the incoming particle is localized in a wave packet centered at a distance $y_{m}$ from the center of mass of the target.

Clearly, this is a special kind of quantized varying field Stark effect. Similar to Stark effect, it is universal, not limited to three-body scattering systems. Namely, the target can be any atomic system that acquired electric moments from any number of internal or environmental conditions. For example, when heated some of the atomic electrons can be excited to higher orbitals [2], the atom becomes deformed and acquired electric moments.

There exists conditions in the E-cat reactor [3] that could produce deformed targets, namely ${ }^{7} \mathrm{Li}$ has nuclear electric quadrupole moment, in addition, it is possible to have deformed atomic ions inside the large molecular complex, for example, ${ }^{7} L i$ in the molecule $\mathrm{LiAlH}_{4}$. The low energy proton released after heating is ideal for the formation of Gailitis resonances.

For long-lived Gailitis resonances $E_{m}<<1 \mathrm{keV}$, which is within the experimental error of nuclear mass data. The explicit inclusion of $E_{m}$ in the energy of the Gailitis resonances is not necessary. To simplify the equations, the energy of the Gailitis resonance are defined by:

$$
\begin{gathered}
\left({ }^{7} L i+p\right)={ }^{7} L i+p+E_{m}>{ }^{7} L i+p=S_{p} \text { of }{ }^{8} B e \\
\left({ }^{A} N i+p\right)={ }^{A} N i+p+E_{m}>{ }^{A} N i+p=S_{p} \text { of }{ }^{A+1} C u
\end{gathered}
$$

Author: Department of Physics and Astronomy, California State

University Long Beach, Long Beach, California, USA.

e-mail:chiyu.hu@csulb.edu 
where $S_{p}$ is the proton separation energy measured from the ground state of atom with mass number $A+1$. Section II presents the fusion process

$$
\left({ }^{7} L i+p\right) \stackrel{\text { fusion }}{\longrightarrow}{ }^{8} B e^{*} \stackrel{\text { decay }}{\longrightarrow} 2 \alpha+\text { kinetic energy. }
$$

Section III will discuss the possibility of the fusion process

$$
\left({ }^{A} \mathrm{Ni}+p\right) \stackrel{\text { fusion }}{\longrightarrow}{ }^{A+1} \mathrm{Cu} \stackrel{\text { decay }}{\longrightarrow}{ }^{A+1} \mathrm{Ni}+\beta^{+}+\bar{\nu}+\text { kinetic energy. }
$$

II. Gailitis Resonance Induced Fusion into THE Giant OVERLAPPING Compound Nuclear Resonances ${ }^{8} B e^{*}$ With Angular Momenta and Parities $I=0^{+}$AND $I=2^{+}$

The ${ }^{8} \mathrm{Be}$ nuclear energy levels have been investigated extensively (see refs [4] and [5] and specific experimental references herein). We are interested in the compound nuclear energy region above the energy level ${ }^{7} L i+p=S_{p}$. There are two sharp resonances located at 0:44 MeV and 2:22 MeV above $S_{p}$. The decay of the first resonance are accompanied by two separate $\gamma$-rays with energies 14:8 MeV and 17:6 MeV, respectively. The second decays only into ${ }^{7} \mathrm{Be}+n$. The energy levels of the these resonances lie much too hight above $S_{p}$ to overlap with the energy of a Gailitis resonance.

Eq. (3) shows that it is only slightly above $S_{p}$. The much investigated [5] giant overlapping resonances with angular momentum and parities $I=0^{+}$and $I=2^{+}$ cover the whole region above and even below the $S_{p}$ energy level according to Ref. [5]. The energy of the Gailitis resonance Eq. (3) can only overlap with that of these Giant resonances investigated in Ref. [5]. The giant resonances can only decay directly into two $\alpha$ particles.

Furthermore, it is easy to demonstrate that the angular momentum coupling of the ${ }^{7} \mathrm{Li}$ nuclei with $I=3 / 2^{-}$and a $p$-state proton with $l=1$, spin $1 / 2$ can produce Gailitis resonances with both $I=0^{+}$and $I=2^{+}$. Despite the vast difference in sizes between the Gailitis resonance and the giant nuclear resonances, the process represented by Eq. (5) conserve energy, angular momentum and parities. The $\alpha$ particle produced by (5) has kinetic energy slightly larger than half of $S_{p}$, that is 8:63 MeV. This energy is much too low to penetrate any nuclei or match any of the energies of the compound nuclear resonances in $N i$ [6]. These results are consistent with the experimental data from the E-cat reactor.

\section{ili. Gailitis Resonances Induced Fusion InVOLVING ${ }^{A} \mathrm{Ni}$ ISOTOPES}

Eq. (6) shows that the complete information of the compound nuclear resonances above proton separation energy of ${ }^{A+1} C u$ are needed in order to satisfy all conservation requirements for these fusion processes. Despite of incomplete information, such as angular momenta and parity of nuclear resonances, the accurate tables of atomic mass energies for all ground states needed in Eq. (6) are readily available in the vast tables of Ref. [7]. From these tables the possibility of Gailitis resonance formation similar to that discussed in Sec. II can be asserted. The conservation of energy in the process represented by Eq. (6) can be established. Of course, it is important to keep in mind that if more nuclear energy levels becomes available, there could be more processes in between or more selection rules must be applied.

The tables provide atomic masses (that's including all the electron as well as nuclear masses) in terms of mass excess $\Delta=M-A, A$ is the mass number. That is $A=Z+N, Z$ the number of proton, $N$ is the number of neutron with $\Delta\left(C_{12}\right)=0$, the unit of $\Delta$ is MeV.

$$
{ }^{A+1} S_{p}={ }^{A} \Delta+\Delta_{H}-{ }^{A+1} \Delta
$$

is the proton separation energy of ground state atom with $A+1$.

$$
{ }^{A+4} S_{\alpha}={ }^{A} \Delta+{ }^{\Delta}{ }^{4} H e-{ }^{A+4} \Delta
$$

is the $\alpha$ particle separation energy of ground state atom with $A+4$.

In table I, $S_{p}$ and $S_{\alpha}$ for a number of ground states $C u$ isotopes are listed. They are calculated using Eqs. (7) and (8), respectively. They are the energy levels in $\mathrm{MeV}$ measured from the ground states copper isotopes respectively.

Table I displays the striking closeness between the two energy levels of $S_{\alpha}$ and $S_{p}$ for all copper isotopes listed and $S_{\alpha}-S_{p}$ changes sign at ${ }^{62} \mathrm{Cu}$. This is unparalleled by other elements in the neighborhood of copper [7], suggesting overlapping compound nuclear resonances similar to that found in Section II. That in turn, suggests the possibility of Gailitis resonances induced fusion of Eq. (6).

Table 2-5 present the details of the energy conservation process following the fusion induced by the Gailitis resonances $\left(p+{ }^{A-1} N i\right)$. 
Had the above process continuing, after emitting numerous low energy $\gamma$-rays, $\beta^{ \pm}$- rays and neutrinos, the process would terminate at the two stable copper elements ${ }^{63} \mathrm{Cu}$ and ${ }^{65} \mathrm{Cu}$. However, one notices that significant compound nuclear energy level structure changes beginning for copper after $A \geq 62$ in table I. It is also noticed that the nuclear binding energy per nucleon for ${ }^{62} \mathrm{Ni}$ of $8: 564 \mathrm{MeV}$ is the largest among all $\mathrm{Ni}$ isotopes, that of the ${ }^{63} \mathrm{Cu}$ of $8: 517 \mathrm{MeV}$ is also the largest among the copper isotopes. A more compact nucleus results in a more compact atom.

As a result, the atoms are not as easy to deform under stress. The experiment results in Ref. [3] strongly suggest that the Gailitis resonance formation process $\left(p+{ }^{62} \mathrm{Ni}\right) \rightarrow{ }^{63} \mathrm{Cu}$ failed such that the processes beginning from Table $\|$ terminate at Table $\mathrm{V}$. Consequently, the isotope $N i^{62}$ accumulate with time at the expense of all other stable isotopes of $N i$.

\section{Discussion}

According to one of the E-cat reactor experiment carried out in Ref. [3], after 32 days of run, the most noticeable changes between the initial (fuel) and final (ash) isotopic compositions are listed in table VI. It is noticed that

a) There is a drastic \% decrease in ${ }^{7} \mathrm{Li}^{+}$.

b) No stable $Z n$ isotopes in the ash.

c) There is a drastic \% increase in ${ }^{62} N i^{+}$at the expense of all the other $N i$ isotopes.

d) No $\gamma$-rays are detected $50 \mathrm{~cm}$ away from the E-cat reactor.

Possible theoretical explanations:

- There are two possibilities that $\left({ }^{7} L i+p\right)$ resonance can be supported. First, the atomic electrons can acquire electric moment under external stress. Second, it is known that ${ }^{7} \mathrm{Li}$ nuclei posses electric quadruple moment. Sec. II successfully matched this Gailitis resonance with the giant compound nuclear resonance in ${ }^{8} B e^{*}$. The $\left({ }^{7} L i+p\right)$ has much longer life time [1] that facilities the formation of ${ }^{8} B e^{*}$. The only decay path of ${ }^{8} B e^{*}$ is two of $\alpha$ particles, each with an $8.63 \mathrm{MeV}$. These processes deplete both ${ }^{7} \mathrm{Li}$ and proton in the fuel, replace them with matching number of $8.63 \mathrm{MeV} \alpha$ particles.

- The energy of the $\alpha$ particle produced in part a) is much too low to match that of the compound nuclear resonates in $N i^{*}$ [6]. They are unable to penetrate the $N i$ nucleus. That could explain the lack of stable $Z n$ isotopes in the ash. Thus the energy of the $\alpha$ particle will dispense into heat after multiple collisions.

- The precise condition for the formation of Gailitis resonances in ${ }^{A} N i+p$ systems is not available at the present time due to the lack of experimental data on compound nuclear resonances above the proton separation energy level in ${ }^{A+1} C u$. The possibility of Gailitis resonance formation is based on circumstantial evidences suggested by the systematics of nuclear masses represented by Table I. Tables II-V evolve using the formation of $\left({ }^{A} N i+p\right)$ and the conservation of energies only. All unknown nuclear energy levels and selection rules due to conservation of parity and nuclear angular momentum are ignored. For example, in Tables II and IV, the $\gamma$-rays, direct transition from the compound nuclear resonant states ${ }^{29} \mathrm{Cu}^{*},{ }^{31} \mathrm{Cu}$ to the ground states of ${ }^{29} \mathrm{Cu},{ }^{31} \mathrm{Cu}$ respectively may not even be possible due to selection rules. The theory of accumulation of ${ }^{62} \mathrm{Ni}$ in the ash depends on future experiment data on nuclear energy level measurements both above and below the proton separation energy.

- Based on energy consideration only, it can be summarized as following: The majority of the released energy eventually turns into heat. The majority of the energy escaped the reactor comes from neutrinos and antineutrinos that accompanying each $\beta^{ \pm}$decay. The numerous $\beta^{+}$rays eventually annihilate with electrons. They produce most of the $\gamma$-rays with an average energy of the order of 0:511 $\mathrm{MeV}$. Can some of the $\gamma$-rays escape the reactor? Certainly. Can they be detected outside the reactor $50 \mathrm{~cm}$ away? This question can be address with more information on the experiment details [8].

\section{References Références Referencias}

1. Chi Yu Hu, David Caballero, Long-range correction in positron-hydrogen scattering system near the threshold of $\operatorname{Ps}(n=2)$ formation, Journal of Modern Physics, 4, 622-627, May 2013, doi:10.4236 /jmp2013.45090 published online May 2013 (http://www.scirp.org/journal/jmp).

2. D. R. Inglis, The energy levels and structure of light nuclei, Review of Modern Physics, 25, 390 (1953), Appendix II, p. 443.

3. Giuseppe Levi, Evelyn Foschi, Bo H"oistad, Roland Petterson, Lars Tegner and Hanno Essen, Observation of abundant heat production from a reactor device and of isotopic changes in the fuel, unpublished, The reactor device is known as E-cat reactor.

4. F. Ajzenberg and T. Lauritsen, The energy levels of light nuclei, Review of Modern Physics, 24, 321 (1952).

5. D. R. Inglis, Theory of Lithium Two-alpha Reactions, 1. Angular Distribution of ${ }^{7} \operatorname{Li}(p, \alpha) \alpha$, Phys. Rev. 74, 21 (1948).

6. S. N. Ghoshal, An Experimental Verification of the Theory of Compound Nucleus, Phys. Rev. 80, 939 (1950).

7. G. Audi, A. H. Wapstra and C. Thibault, The AME 2003 atomic mass evaluation, Nuclear Physics A, 729, (2003) 337-676. 
8. Robley D. Evans, The Atomic Nucleus, p. 725, McGraw-Hill, 1955.

Table /: The ground states atoms ${ }^{A} C u, A=59-65$, their life-times, mass excess $\Delta$ in MeV, proton separation energy in $\mathrm{MeV}$, and $\alpha$ particle separation energy in $\mathrm{MeV}$ are listed, values are taken directly from Ref. [7]

\begin{tabular}{|l|ccccccc|}
\hline \hline & ${ }^{59} \mathrm{Cu}$ & ${ }^{60} \mathrm{Cu}$ & ${ }^{61} \mathrm{Cu}$ & ${ }^{62} \mathrm{Cu}$ & ${ }^{63} \mathrm{Cu}$ & ${ }^{64} \mathrm{Cu}$ & ${ }^{65} \mathrm{Cu}$ \\
\hline \hline life time & $81.1 \mathrm{~s}$ & $23.7 \mathrm{~m}$ & $3.333 \mathrm{~h}$ & $9.67 \mathrm{~m}$ & stable & $12.7 \mathrm{~h}$ & stable \\
\hline$\Delta$ & -56.357 & -58.344 & -61.984 & -62.798 & -65.579 & -65.424 & -67.264 \\
\hline$S_{p}$ & 3.419 & 4.477 & 4.801 & 5.866 & 6.122 & 7.201 & 7.453 \\
\hline$S_{\alpha}$ & 4.755 & 4.730 & 5.064 & 5.377 & 5.776 & 6.200 & 6.790 \\
\hline$S_{\alpha}-S_{p}$ & 1.336 & 0.253 & 0.263 & -0.489 & -0.346 & -1.001 & -0.663 \\
\hline
\end{tabular}

Table // : $\mathrm{A}=59, T_{\beta+\nu}$ is kinetic energy of $\beta^{ \pm}$plus the energy carried away by neutrino

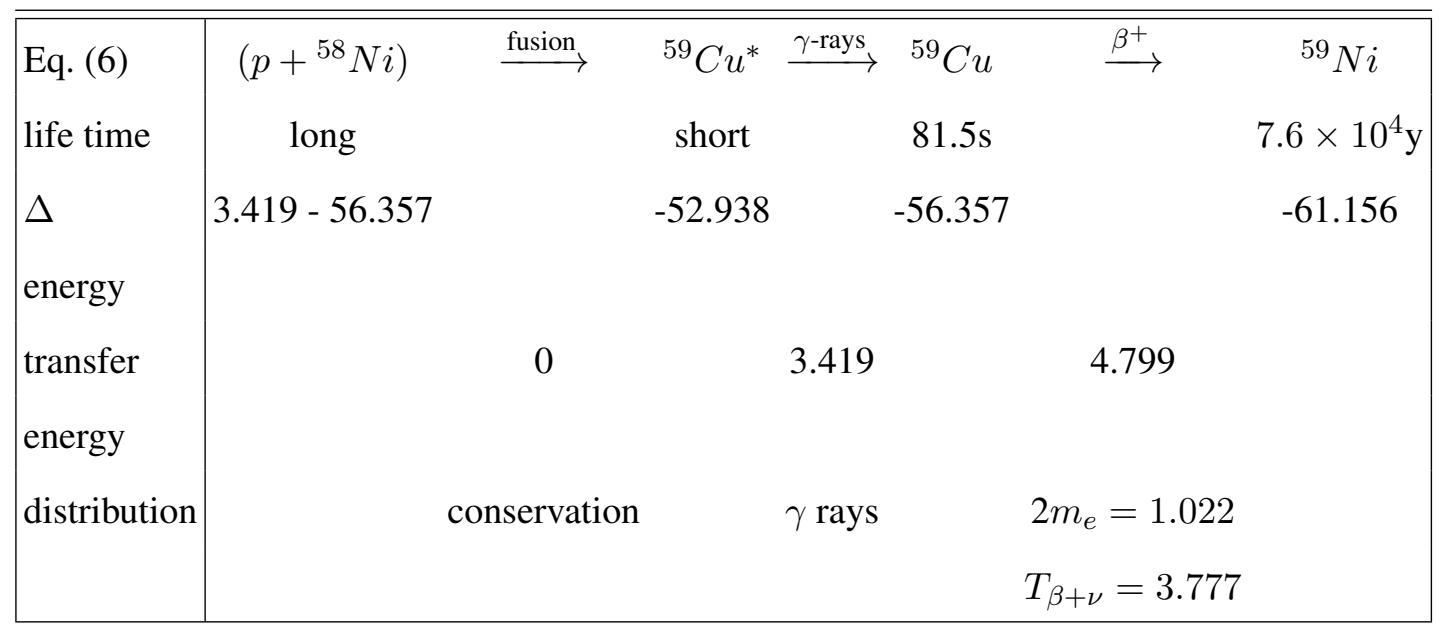

Table /II : $\mathrm{A}=60, T_{\beta+\nu}$ is kinetic energy of $\beta^{ \pm}$plus the energy carried away by neutrino

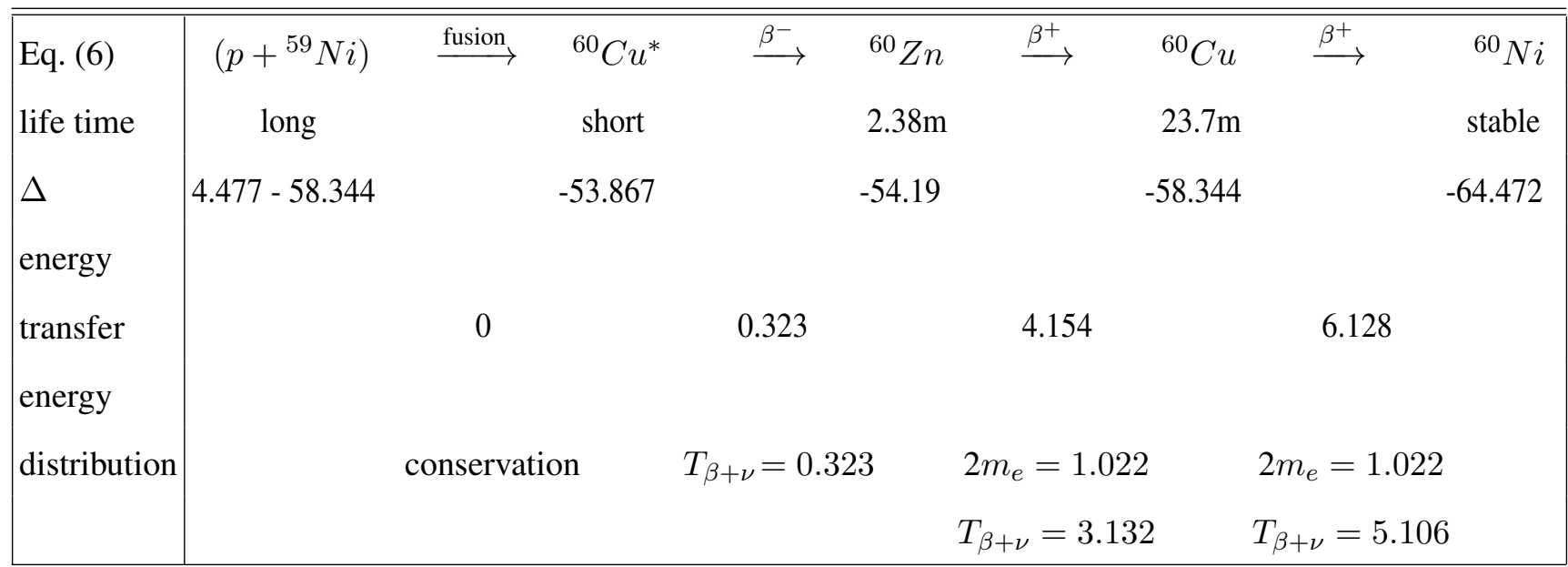


Table $\mathrm{V}: \mathrm{A}=61, T_{\beta+\nu}$ is kinetic energy of $\beta^{ \pm}$plus the energy carried away by neutrino

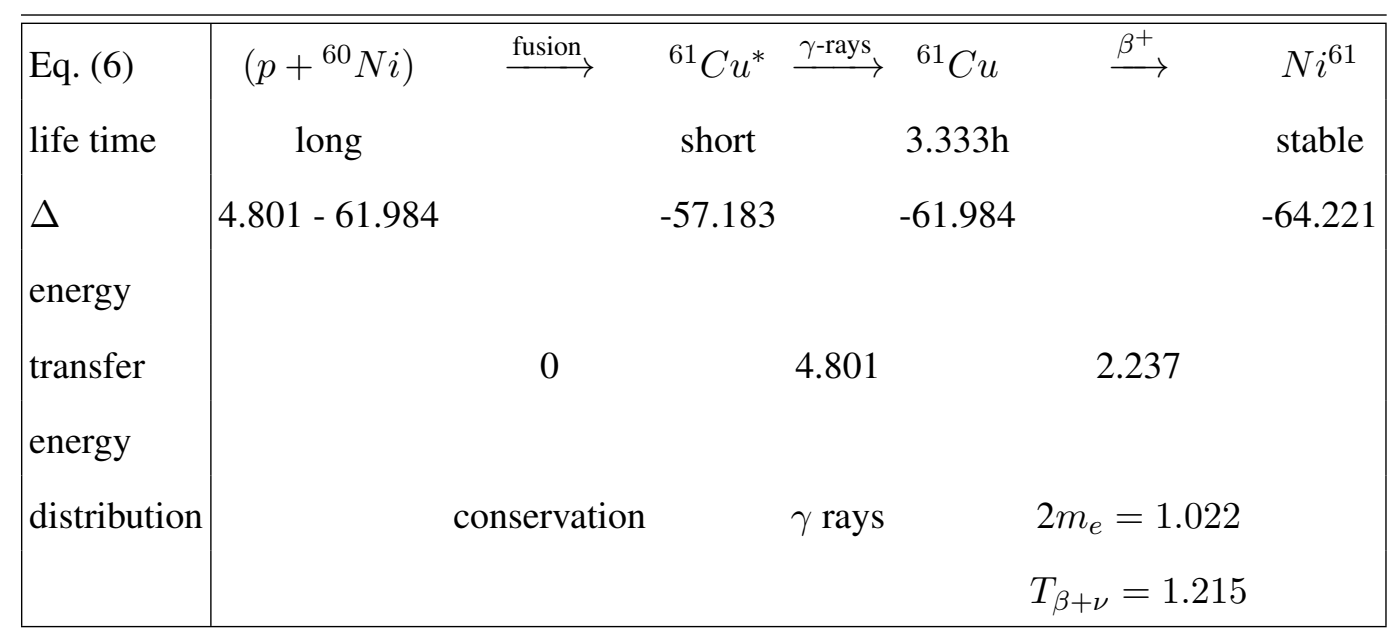

Table $\mathrm{V}: \mathrm{A}=62, T_{\beta+\nu}$ is kinetic energy of $\beta^{ \pm}$plus the energy carried away by neutrino

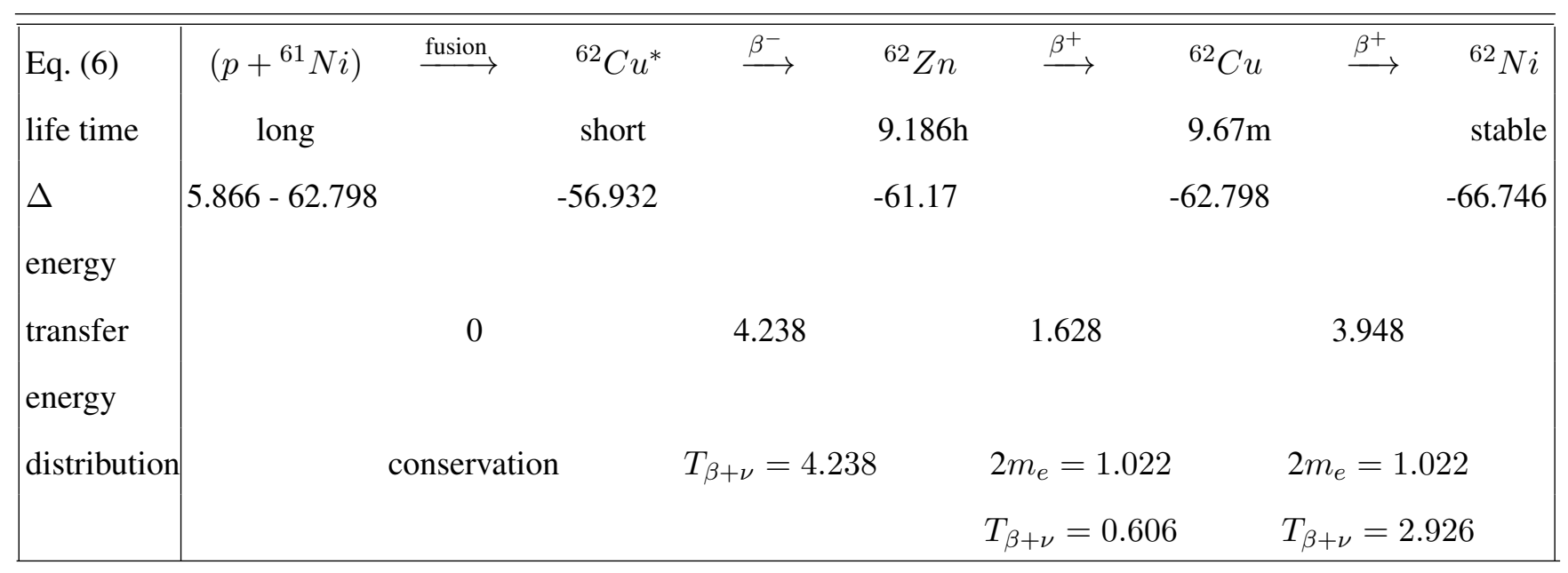

Table VI : Changes in isotopic composition

\begin{tabular}{|c|cccccccc|}
\hline & ${ }^{6} \mathrm{Li}^{+}$ & ${ }^{7} \mathrm{Li}^{+}$ & ${ }^{58} \mathrm{Ni} i^{60} \mathrm{Ni}^{+}$ & ${ }^{61} \mathrm{Ni} i^{+62} \mathrm{Ni}^{+}$ & ${ }^{64} \mathrm{Ni} i^{+}$ \\
\hline fuel & $8.6 \%$ & $91.4 \%$ & $67 \%$ & $26.3 \%$ & $1.9 \%$ & $3.9 \%$ & $1 \%$ \\
ash & $92.1 \%$ & $7.9 \%$ & $0.8 \%$ & $0.5 \%$ & $0 \%$ & $98.7 \%$ & $0 \%$ \\
\hline
\end{tabular}




\title{
Formulate Equations for Reciprocity Vector and Scalar of Some Mechanical and Electrical Quantities
}

\author{
By Faisal A. Mustafa \\ Babylon University, Iraq
}

Abstract- This study deals a new concepts of some physical reciprocally quantities which are vectors or scalars and its relationship with the fundamental quantities. The reciprocal of kinematics dimension quantities of moved particle for linear, rotational and circular movement are resulted the quantities that so called the time quantities such as time velocity, time acceleration. Other derivative quantities were derived from the principle equations to produce reciprocal movement equations. For dynamical mechanics, the quantities were derived from the principle equation such as time force, momentum, torque, energy,... ect. Also some reciprocal quantities due to electricity equations include the reactance, electric current, conductivity, ....ect, and its derivative quantities are introduced time current density, time power.

Keywords: reciprocal of velocity, conductivity, reciprocal current, inverse quantity, reciprocal, time electric field.

GJSFR-A Classification : FOR Code: 240402

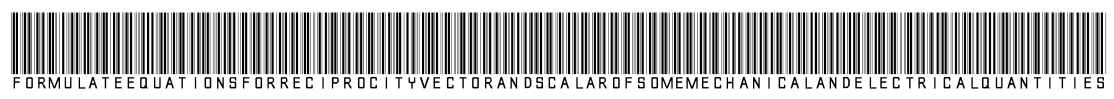

Strictly as per the compliance and regulations of:

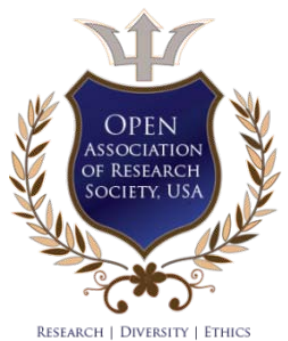

(c) 2015. Faisal A. Mustafa. This is a research/review paper, distributed under the terms of the Creative Commons AttributionNoncommercial 3.0 Unported License http://creativecommons.org/licenses/by-nc/3.0/), permitting all non commercial use, distribution, and reproduction in any medium, provided the original work is properly cited. 


\title{
Formulate Equations for Reciprocity Vector and Scalar of Some Mechanical and Electrical Quantities
}

\author{
Faisal A. Mustafa
}

\begin{abstract}
This study deals a new concepts of some physical reciprocally quantities which are vectors or scalars and its relationship with the fundamental quantities. The reciprocal of kinematics dimension quantities of moved particle for linear, rotational and circular movement are resulted the quantities that so called the time quantities such as time velocity, time acceleration. Other derivative quantities were derived from the principle equations to produce reciprocal movement equations. For dynamical mechanics, the quantities were derived from the principle equation such as time force, momentum, torque, energy,...ect. Also some reciprocal quantities due to electricity equations include the reactance, electric current, conductivity, ...ect, and its derivative quantities are introduced time current density, time power. The results show that the concept of reciprocal of some quantities describes the quantity from two faces, first the principle quantities as were known, which depend on time and second the reciprocal of this quantity, that independent on time and gives a new concept to the same quantity, describe the motion of particle or field. This will add a complete meaning of movement of a particle, if a particle is a moving neutral or charged particle, or intrinsic quantity.
\end{abstract}

Keywards: reciprocal of velocity, conductivity, reciprocal current, inverse quantity, reciprocal, time electric field.

\section{InTRODUCTION}

$\mathrm{M}$ ost the physical laws are dependant on time. The concept of the reciprocally physical quantity, gives another meaning, but with the same concept of moving particle or group of particles, for example: the reciprocal of velocity for kinematics ( with no inertia) and for dynamics movement give another concept. (Mushfiq, 2009) defined as well slowness $v$ as reciprocal of $v ; \dot{v}=1 / v=t / x$, therefore, objectivity demands that motion described in terms of slowness that should be as valid as the description in terms of velocities, its postulate in kinematics is symmetry under reciprocal inversion of velocities or the motion is invariant under inversion $v \rightarrow 1 / v$ and vice versa [1]. Slowness must form a group, difference between two slowness is slowness, therefore slowness must be discrete[2]. ( Md Shah Alam, et al.2005) Applied the mixed numbers algebra( sum of scalar and vector) in quantum mechanics, electrodynamics and

Author: Laser Physics Department/College of Science for Women, Babylon University-Iraq.e-mail:albassam1799@yahoo.co.uk special relativity as a complete tools[3].( Mushfiq,2012) have observed a reciprocal relation between Planck's hypothesis and Einstein's postulate (special relativity). Particle velocity and de Broglie wave velocity are also reciprocally related[4]. The longitudinal component of the velocity of a particle at or near a glacier surface, its position is a function of time being term its trajectory velocity distribution are studied by (L.A. Rasmussem, 1983)[5].The reciprocal relation give a correspondence between discrete and continuous quantities[4]. Movement constitutes a set defined by intrinsic relativity, reciprocity, and simulation of translation and rotation of all moving entities, the soveons. Soveon, elements and subsets, have angular and linear velocities[6].

In pure geometry the theories of similar (reciprocal theorem of Betti and Raleigh) reciprocal and inverse figures (reciprocal diagram) have led to many extensions of science (e.g. the refractive index being proportional to the velocity or the reciprocal of velocity) [7]. Attenuation coefficient is measured using units of reciprocal length. If the capsular theory was taken, it will use the reciprocal of velocity as a multiplier instead of the velocity itself. For simplicity, the variability of the time $\mathrm{t}(\mathrm{x})$ is elapsing between timing events, but the time velocity and reciprocal of velocity are the same[8] The concepts generalize to time - varying and to vector valued Morse functions [9]. It sometimes uses the reciprocal lattice for crystal structure. It should now be clear that the direct lattice, and its reticular planes, are directly associated (linked) with the reciprocal lattice. (Lima Siow) formulated equivalent principle that the kinetic acceleration is equal to the potential acceleration $\left(d^{2} r / d^{2}=-d \Phi / d r\right)$ [10] Lorson explains, in the equation of motion, time is the reciprocal of space and space is the reciprocal of time. This leads to a new concept of motion, which Larson calls scalar motion. Larson's idea that time and space are reciprocals is difficult to understand in the context of the conventional space time framework as if to say that the march of time is the reciprocal of extension space, which we ordinarily think of as a container of matter. It is much easier to grasp when we considers a theoretical universe of motion in which the only significant physical quantity is the magnitude of that motion, measured as speed or velocity [11]. 
As noted earlier, in the RST's (reciprocal state of time) postulated three-dimensional motion, threedimensional time-motion is the inverse of threedimensional space-motion. But it is important to remember that space-motion has no direction in time, just as time-motion has no direction in space. Likewise, in the equation of time-motion, $\mathrm{v}^{\prime}=\mathrm{t} / \mathrm{s}$, $\mathrm{t}$ has direction in time, but $s$ has no direction in time and is a scalar value. Larson summarizes this concept as follows: In the equations of motion in space, time is scalar. In the equations of motion in time, space is scalar and In the equations of motion in space-time, both space and time are scalar. However, in certain situations they are distinguished, as follows[11]. The term reciprocal refers to the key concept of the RST that reflects the postulated relationship of space and time as reciprocals of each other in the definition of motion. This strict study does shed more light into understanding the basic conceptual and theoretical framework on which the concepts are more complicated to other fields of physics. The moving phenomenon of particle are described from two sides dimensionally and timely which have a second new formulas for mechanical and electrical equations. From the practical point of view, it is important because it provides techniques, which can be used in almost any areas of pure and applied researches due to motions.

\section{THEORETICAL}

Suppose $\mathrm{y}_{\mathrm{x}}$ is a physical vector quantity, and $\mathrm{y}_{\mathrm{t}}$ is the reciprocity, so physical quantity vector is equal to the reciprocal of another quantity which have the same direction and another concept of the same phenomena movement:

$$
\overrightarrow{y_{x}}=-\frac{\hat{x}}{y_{t}}
$$

is the unit vector of the quantity $y_{t}, \hat{x}$ Where

The negative sign indicates to comparison between two or more values of physical quantity with respect to each other, that the more negativity value have the less value.

$$
\vec{y}_{x}=\frac{d \vec{x}}{d t}
$$

suppose

So

$$
\vec{y}_{x}=-\hat{x} \frac{d t}{d x}
$$

Equation 2 can be applied for all vector quantities related to time. If the physical quantity is scalar, thus the reciprocal of any scalar quantity $(P)$ is equal to another concept of the scalar quantity or: $(Q)$

$$
Q=\frac{1}{P}
$$

Equation 3 can be applied to any physical scalar quantity by taken its reciprocity.

\section{a) Reciprocal of Some Physical Quantities \\ i. The Reciprocal of Velocity}

It states that the reciprocal of kinetic dimension velocity is equal to the gradient of time or time velocity, which conventionally is expressed as [12]:

$$
\frac{\hat{\mathrm{u}}}{v_{r}}=-\operatorname{grad} \mathrm{t}
$$

or $-\left(\hat{x} \frac{\partial t}{\partial x}+\hat{y} \frac{\partial t}{\partial y}+\hat{z} \frac{\partial t}{\partial z}\right)$ Where the R.H.S. of the equation is equivalent to

$$
\overrightarrow{v_{t}}=-\frac{\hat{\mathrm{u}}}{v_{r}}
$$

such that $\mathrm{u}$ is a unit vector of vr or vt and the absolute value of equation (5) leads to the formula $v_{\mathrm{r}}=1 / v_{\mathrm{t}}$

The negative sign of eq. (5) is placed to compare two or more numerical values of particle velocity. In other wards the negative numerical value decreases as number increase. The variable $v_{t}$ is defined as the reciprocal of dimension velocity or velocity of time in any units (in units: $\mathrm{scm}^{-1}, \mathrm{sm}^{-1}$, or $\left.\mu \mathrm{sm}^{-1}, \ldots\right)$.

The change of the time speed or velocity per unit displacement of an object give us another concept of acceleration called time acceleration.

To evaluate the time acceleration, multiply eq.

(4) by the grad operator this yields[11]:

$$
\nabla \cdot v_{\mathrm{t}}=-\nabla \cdot \nabla \mathrm{t}
$$

or

$$
\frac{d v_{t}}{d r}=-\nabla^{2} t
$$

such that the left-hand side of equation (7) is a scalar quantity called the acceleration of time $\left(a_{t}\right)$ (in units $s$

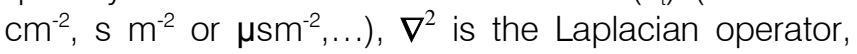
however in three dimension equation (7) becomes:

$$
a_{t}=\frac{\partial^{2} t}{\partial x^{2}}+\frac{\partial^{2} t}{\partial y^{2}}+\frac{\partial^{2} t}{\partial z^{2}}
$$

The kinematical time acceleration in one dimension, becomes: 


$$
\begin{aligned}
& a_{t x}=\frac{d^{2} t}{d x^{2}} \\
& a_{t x}=\frac{d v_{t}}{d x}
\end{aligned}
$$

The new groups of equations with respect to time motion for linear and rotation motion are viewed in[12] which results the important equation:

$$
a_{t}=-\frac{2 v_{t_{0}} v_{t_{1}}\left(v_{t_{1}}-v_{t_{0}}\right)}{t\left(v_{t_{1}}+v_{t_{0}}\right)}
$$

And

$$
v_{t 1}=v_{t 0} \frac{a_{t} x}{a_{x} t}
$$

For dynamic movement, the change of time momentum to displacement is equal to time force that is non-vector in unit (gm.s. $\mathrm{cm}^{-2}$ or $\left.\mathrm{kg} . \mathrm{s} . \mathrm{m}^{-2}\right)$. It is like a second Newton's law that given by:

$$
F_{t}=\frac{d p_{t}}{d s}=\frac{d\left(m v_{t}\right)}{d s}=\frac{m d v_{t}}{d s}=m a_{t}
$$

Table 1 refer to one- dimensional of physical time quantities for kinematical and dynamical movement with different symbols and units. When the numerical values is substituted in the dimensional and time equations, the produced values are coincidence to interpretation the physical phenomena in two side dimensionally and timely.
Ex: Dynamic particle have data: $\mathrm{v}_{1 \mathrm{x}}=3 \mathrm{cms}^{-1}, \mathrm{x}=18 \mathrm{~cm}$, $\mathrm{a}_{\mathrm{x}}=2 \mathrm{cms}^{-1}, \mathrm{~m}=5 \mathrm{gm}$, find : $\mathrm{v}_{2 \mathrm{x}}, \mathrm{t}, \mathrm{v}_{2 \mathrm{t}}, \mathrm{a}_{\mathrm{t}}, \mathrm{F}_{\mathrm{t}}, \mathrm{w}_{\mathrm{t}}, \mathrm{p}_{\mathrm{t}}$.

Solution:

$\mathrm{v}_{2 \mathrm{x}}=9 \mathrm{cms}^{-1}, \mathrm{t}=3 \mathrm{~s}, \mathrm{v}_{2 \mathrm{t}}=0.111 \mathrm{scm}^{-1}, \mathrm{a}_{\mathrm{t}}=0.0123 \mathrm{scm}^{-2}$, $\mathrm{F}_{\mathrm{t}}=0.0615 \mathrm{gm} . \mathrm{s} . \mathrm{cm}^{-2}, \mathrm{~W}_{\mathrm{t}}=0.1845 \mathrm{gms}^{2} \mathrm{~cm}^{-2}, \mathrm{p}_{\mathrm{t}}=$ $0.8333{\mathrm{gm} . \mathrm{scm}^{-1}}^{-1}$

\section{ii. The Lorentz - Einstein transformations}

The relative uniform translational and rotational time velocities are derived from the known dimension velocity equations. The Lorentz - Einstein transformations are converted by the principle formula (reciprocal) [13].

The three equations give another Galilean rule of a particle as measured with time velocity by two observers in relative translational motion is given $b$

$$
V_{t z^{\prime}}^{\prime}=V_{t z} \quad V_{t y^{\prime}}^{\prime}=V_{t y}, \quad V_{t x^{\prime}}^{\prime}=\frac{V_{t x} V_{t x}}{V_{t x}-V_{t x}}
$$

For two observers have the same time acceleration the equations are[14]:

$$
a_{t x}^{\prime}=\frac{V_{t x}{ }^{2} a_{t x}{ }^{\prime \prime}-v_{t x}{ }^{2} a_{t x}}{\left(v_{t x}-V_{t x}\right)^{2}}, a_{t y}{ }^{\prime}=\frac{d V_{t y}}{d y^{\prime}} a_{t z}{ }^{\prime}=\frac{d V_{t z}}{d z}
$$

The dimension and time velocity of $A$ as measured by o has components[14]:

$$
V_{x}=\frac{d x}{d t}, V_{y}=\frac{d y}{d t}, V_{z}=\frac{d z}{d t} \quad V_{t x}=\frac{d t}{d x}, V_{t y}=\frac{d t}{d y}, V_{t z}=\frac{d t}{d z}(15)
$$

Similarly, the components of distance and time velocities of $A$ as measured by $o$ / are

$$
V_{x^{\prime}}^{\prime}=\frac{d x^{\prime}}{d t^{\prime}}, V_{y^{\prime}}^{\prime}=\frac{d y^{\prime}}{d t^{\prime}}, V_{z^{\prime}}^{\prime}=\frac{d z^{\prime}}{d t^{\prime}} V_{t^{\prime} x^{\prime}}^{\prime}=\frac{d t^{\prime}}{d x^{\prime}}, \quad V_{t y}=\frac{d t}{d y} V_{t z}=\frac{d t}{d z}
$$

\section{iii. Reciprocal of Mass Density and pressure}

Specific volume is the volume occupied by a unit of mass of a material[15]. The specific volume of a substance is equal to the reciprocal of its mass density. Specific volume may be expressed in $\mathrm{m}^{3} \mathrm{~kg}^{-1}$ :

$$
v=\frac{V}{m}=\frac{1}{\rho}
$$

where, $V$ is the volume, $m$ is the mass and $\rho$ is the density of the material.

For an ideal gas, pressure is equal to the reciprocal of volume with constant $=\mathrm{NkT}[16]$ :

$$
p=\frac{c}{V}
$$

Table 1 : The derived mechanical time quantities and its description and equations

\begin{tabular}{|c|c|c|c|c|}
\hline Derived Time quantity & Description & Sl units & equation & Comments \\
\hline Time Angular Velocity & change in time angular velocity per unit angle & $\mathrm{s} \mathrm{rad}^{-1}$ & $\omega_{t}=\frac{d t}{d \theta}$ & $\begin{array}{c}\mathrm{scalar} \text { or } \\
\text { pseudovector }\end{array}$ \\
\hline Time Angular Acceleration & Product of an object's mass and time velocity & $\mathrm{s} \mathrm{rad}^{-2}$ & $\alpha_{t}=\frac{d \omega_{t}}{d \theta}$ & $\mathrm{scalar}$ \\
\hline Time Linear Momentum & $\begin{array}{c}\text { Measure of the extent and direction of object } \\
\text { rotates at time. }\end{array}$ & $\mathrm{kg} \mathrm{sm}^{-1}$ & $P_{t}=m v_{t}$ & Vector, extensive \\
\hline
\end{tabular}




\begin{tabular}{|c|c|c|c|c|}
\hline Time Angular Momentum & $\begin{array}{l}\text { The cause of time acceleration, acting on an } \\
\text { object. }\end{array}$ & $\mathrm{kgs}^{3}$ & $L_{t}=m t^{2} \omega_{t}$ & $\begin{array}{l}\text { conserved quantity, } \\
\text { pseudo vector }\end{array}$ \\
\hline Time Impulse & $\begin{array}{l}\text { Inertia of an object with respect to angular } \\
\text { acceleration. }\end{array}$ & $\mathrm{N}_{\mathrm{t}} \mathrm{s}$ & $I_{m p}=F_{t} t$ & scalar \\
\hline Time Torque (moment of force) & $\begin{array}{l}\text { Energy dissipated by a time force moving over } \\
\text { a time moment, scalar product of the time } \\
\text { force and the time. }\end{array}$ & Nt m & $\tau_{t}=r F_{t}$ & tensor \\
\hline Time Work & $\begin{array}{c}\text { The capacity of a body or system to do time } \\
\text { work. }\end{array}$ & $\begin{array}{l}J_{t}=k g \\
s^{2} m^{-2}\end{array}$ & $W_{t}=F_{t} t$ & scalar \\
\hline Time Kinetic Energy & The rate of change in energy over time. & $\begin{array}{l}\text { Joule } \\
(\mathrm{J}=\mathrm{kg} \\
\left.\mathrm{s}^{2} \mathrm{~m}^{-2}\right)\end{array}$ & $E_{t}=\frac{1}{2} m v_{t}^{2}$ & $\begin{array}{l}\text { extensive, scalar, } \\
\text { conserved quantity }\end{array}$ \\
\hline Time potential Energy & The conserved energy over time & $\begin{array}{l}\left(\mathrm{Jt}=\mathrm{kgs}^{2}\right. \\
\left.\mathrm{m}^{-2}\right)\end{array}$ & $E_{p t}=m g_{t} t$ & scalar \\
\hline Time Pressure & $\begin{array}{l}\text { Amount of time force exerted per surface } \\
\text { area. }\end{array}$ & $\mathrm{kg} \mathrm{s} \mathrm{m}^{-4}$ & $P_{t}=\frac{F_{t}}{A}$ & intensive \\
\hline Time Weight & $\begin{array}{l}\text { Amount of gravitation force exerted on an } \\
\text { object. }\end{array}$ & $\begin{array}{l}\text { Newton } \\
\mathrm{N}=\mathrm{kg} \mathrm{m} \\
\left.\mathrm{s}^{-2}\right)\end{array}$ & $w_{t}=m g_{t}$ & scalar \\
\hline Time Moment of Inertia & $\begin{array}{l}\text { Product of a time force and the perpendicular } \\
\text { distance }\end{array}$ & $\mathrm{kg} \mathrm{m}^{2}$ & $\mathrm{I}=\mathrm{mr}^{2}$ & tensor \\
\hline Time Power & Amount of time force per unit area. & $\underline{\text { watt }}_{\underline{t}}\left(\mathrm{~W}_{\mathrm{t}}\right)$ & $P_{t}=F_{t} V_{t}$ & $\begin{array}{c}\text { scalar or } \\
\text { pseudovector }\end{array}$ \\
\hline
\end{tabular}

\section{iv. Reciprocal of electric current and conductance}

The law of dimensional electric current is defined as[16]:

$$
I_{x}=\frac{d Q}{d t}
$$

The reciprocal electric current is called time electric current that defined as the rate time to passing unit charge:

$$
I_{t}=-\frac{d t}{d Q}
$$

The negative sign refers to compare more than one value of the current, so :

$$
I_{x}=-I_{t}^{-1}
$$

The absolute value of equation (20) becomes Ix=It-1

This follows the displacement and time current densities $J_{x}$ and $J_{t}$ related to two equations by using amount of electric current flowing through a surface:

$$
J_{x}=\frac{I_{x}}{A}, J_{t}=\frac{I_{t}}{A}
$$

In Amp. $\mathrm{cm}^{-2}$ and $\mathrm{Amp}^{-1} . \mathrm{cm}^{-2}$ respectively.

The reciprocal of electric conductance is equal to resistance (in Ohm), also the reciprocal of impedance is equal to admittance $\left(\mathrm{Ohm}^{-1}\right)$, the reciprocal of dimension electrical conductivity is a measure of how well a material accommodates the transport of electric charge and equal to dimension resistivity or specific resistance:

$$
\sigma_{t}=\frac{1}{\rho_{t}} \quad \text { and } \quad \sigma_{x}=\frac{1}{\rho_{x}}
$$

Dimensional electrical resistance is a measure of the degree to which an electrical component opposes the passage of displacement current. It is the ratio of the potential difference (i.e. voltage) across an electric component (such as a resistor) to the displacement or time current passing through that component:

$$
R_{x}=\frac{V}{I_{x}} \text { and } \quad R_{t}=\frac{V}{I_{t}}
$$

Where $\mathrm{R}_{\mathrm{x}}$ is the resistance of the component. $V$ the voltage across the component, measured in volts, $I_{x}$ and it is the dimension and time current passing through the component, $V$ can either be measured directly across the component or calculated from a subtraction of voltages relative to a reference point. Resistance is thus a measure of the component's opposition to the flow of electric charge. Its reciprocal quantity is electrical conductance measured in siemens. electrical admittance $(1 / Z)$ is equal to reciprocal of electric impedance in Siemens (or) mhos Strength of the electric field[16]: 


$$
\begin{gathered}
E_{x}=-\frac{d V}{d x}\left(\mathrm{Vm}^{-1}\right) \text { (vector field) } \\
R_{t}=\frac{l}{\sigma_{t} A} \quad R_{x}=\frac{l}{\sigma_{x} A} \\
\left.V_{s^{-1}}\right) \quad E_{t}=-\frac{d V}{d t} \\
\frac{E_{t}}{E_{x}}=v_{x}
\end{gathered}
$$

Electrical conductance is a measure of current flows through a material:

siemens $\quad G=\frac{1}{\rho} \quad(29) \quad($ scalar $)\left(\mathrm{S}=\mathrm{A}^{2} \mathrm{~s}^{3} \mathrm{~kg}^{-1} \mathrm{~m}^{-2}\right)$

is Measure for the resistance of an electrical circuit against an alternating current. ohm $(\Omega=\mathrm{kg} \mathrm{m} 2 \mathrm{~A}-2 \mathrm{~s}-3)$ Impedance

$$
\text { (complex scalar) } \quad Z_{x}=\frac{V}{I_{x}}
$$

The degree to which an object opposes the passage of an electric current is called resistance (scalar)

$$
R=\frac{1}{\text { conduc }}
$$

The displacement electric power in watts produced by an dimension electric current $I_{\mathrm{x}}$ consisting of a charge of $Q$ coulombs every $t$ seconds passing through an electric potential (voltage) difference of $V$ is:

$$
\begin{aligned}
& P_{x}=\frac{Q V}{t}=Q E_{t} \\
& P_{t}=\frac{V t}{Q}=\frac{V}{I_{x}}
\end{aligned}
$$

Divide eq.(29) and (30) produce :

$$
\frac{P_{x}}{P_{t}}=I_{x}{ }^{2}
$$

Ex. A charge $6 \mu$ coul. is passing through wire at ${ }_{2} \mu \mathrm{s}$, its length and area is $2 \mathrm{~cm}$ and $10 \mathrm{~cm}^{2}$ respectively ,if the potential difference is 3volt. Calculate $\mathrm{I}_{\mathrm{x}}, \mathrm{I}_{\mathrm{t}}, \mathrm{J}_{\mathrm{x}}, \mathrm{J}_{\mathrm{t}}$,

\begin{tabular}{|c|c|c|c|c|}
\hline $\begin{array}{l}\text { Derived time } \\
\text { quantity }\end{array}$ & Description & SI units & equation & comments \\
\hline $\begin{array}{l}\text { Time Electric Field } \\
\text { Intensity }\end{array}$ & $\begin{array}{l}\text { The time force per charge when placed in the } \\
\text { electric field. }\end{array}$ & $\mathrm{Kg} \cdot \mathrm{sm}^{-2} \mathrm{C}^{-1}$ & $E_{t}=\frac{F_{t}}{Q}$ & scalar \\
\hline $\begin{array}{l}\text { Time Electric Flux } \\
\text { Density }\end{array}$ & Is due to time electric field depend on medium & C. $s^{3} m^{-3}$ & $D_{t}=\varepsilon E_{t}$ & scalar \\
\hline Time Electric Flux & Electric flux in terms of electric flux density & C. $\mathrm{s}^{4} \cdot \mathrm{m}^{-3}$ & $\psi_{t}=\int D_{t} d t$ & scalar \\
\hline Electric Potential & The time work done in displacing the charge by dl. & Volts & $V=\int E_{t} d t$ & scalar \\
\hline Time Power Density & $\begin{array}{c}\text { The potential difference per unit displacement } \\
\text { current. }\end{array}$ & V.s.C.-1 & $P_{t}=V I_{t}$ & scalar \\
\hline $\begin{array}{l}\text { Electric charge } \\
\text { density }\end{array}$ & Amount of electric charge per unit volume. & $\mathrm{C} \mathrm{m}^{-3}$ & $\rho_{v}=\frac{q}{v}$ & intensive \\
\hline Energy density & Amount of energy per unit volume. & $\mathrm{J} \mathrm{m}^{-3}$ & $\rho_{E}=\frac{E}{v}$ & intensive \\
\hline
\end{tabular}
$\sigma^{\mathrm{x}}, \sigma_{\mathrm{t}}, \mathrm{E}_{\mathrm{x}}, \mathrm{E}_{\mathrm{t}}, \mathrm{v}_{\mathrm{t}}, \mathrm{v}_{\mathrm{x}}$.

Solution: $\quad \mathrm{I}_{\mathrm{x}}=3 \mathrm{Amp} . ., \quad \mathrm{I}_{\mathrm{t}}=0.333 \mathrm{Amp}^{-1}$ $, \mathrm{J}_{\mathrm{x}}=0.3 \mathrm{Amp} . \mathrm{cm}^{-2}, \quad \mathrm{~J}_{\mathrm{t}}=0.0333 \mathrm{~A}^{-1} \mathrm{~cm}^{-2}, \quad \sigma_{\mathrm{x}}=0.20 \mathrm{hmcm}^{-}$, ,$\sigma_{\mathrm{t}}=0.0222 \quad$ A. Voltcm ${ }^{-1}, \quad \mathrm{E}_{\mathrm{x}}=1.5$ Volt. $\mathrm{cm}^{-1}, \quad \mathrm{E}_{\mathrm{t}}=1.5$ ${ }^{*} 10^{6}$ Volt. $^{-1}, \mathrm{v}_{\mathrm{t}}=\mathrm{E}_{\mathrm{x}} / \mathrm{E}_{\mathrm{t}}=10^{-6} \mathrm{scm}^{-1}, \mathrm{v}_{\mathrm{x}}=10^{6} \mathrm{cms}$.

The time equations of electric quantities and its derivatives are shown in table 2.

Table 2: represents the description and equations of derived time electric quantities

\section{ili. Results And Discussion}

The principle problem of kinematics is that of determining all characteristics of a particle as a whole or any of particles or bodies [1]. It was noted that the reciprocal of dimension velocity is equivalent to the gradient of elapsed time that is also defined velocity of time. The negative sign of equation (4) means that the effective numerical value of both $v_{x}$ and $v_{t}$ have the same concept for the moving particle. Equation (4) is similar to electrical field equation 
acceleration of equation (7) is analogous to Poisson's equation $\left(\nabla^{2} \varphi=-\rho / \varepsilon\right)[14]$.

Figure 1 indicates any postulated values of $v_{x}$ and $v_{\mathrm{t}}$ that described the symmetry relationship of dimension and time velocities which represent the reciprocal diagram. The negative inverse proportional relation between $v_{\mathrm{r}}$ and $v_{\mathrm{t}}$, have the same kinetic meaning as a numerical value for a particle motion. They are a symmetry about origin. If we have taken any numerical value of $v_{x}$ or $v_{t}$ will have the same numerical velocity meaning, or there are no difference between the new factors related to time and the ordinary factors due to dimension to know the moving values of particle.
Figure 2 describes the relationship between the dimension acceleration and time acceleration of moving particle with initial and final dimension or time velocity by applying equations (11) and (12). The curve tend to the slowness for time acceleration. So when the dimension acceleration increases, the time acceleration decrease in its value and occur the slowness state. Figure 3 shows the relationship between the dimension force and time force, It is found that $F_{t}$ begins to decrease with respect to $F_{x}$, also for dimension and time work describe the same variation as shown in figure 4 . The postulated values were applied for moving particle in straight line by using equations as shown in table 1 .

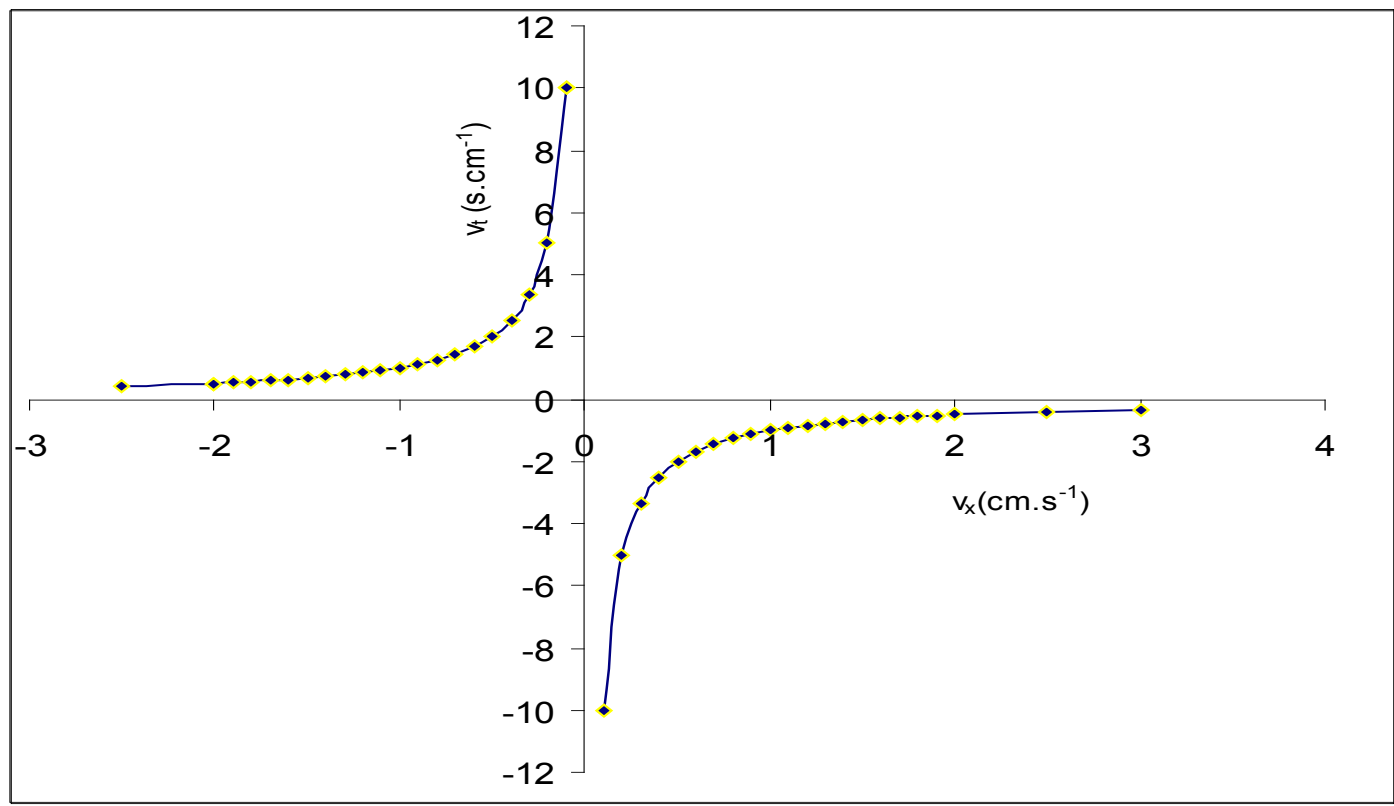

Figure 1: The relationship between velocity and its reciprocal for postulated values.

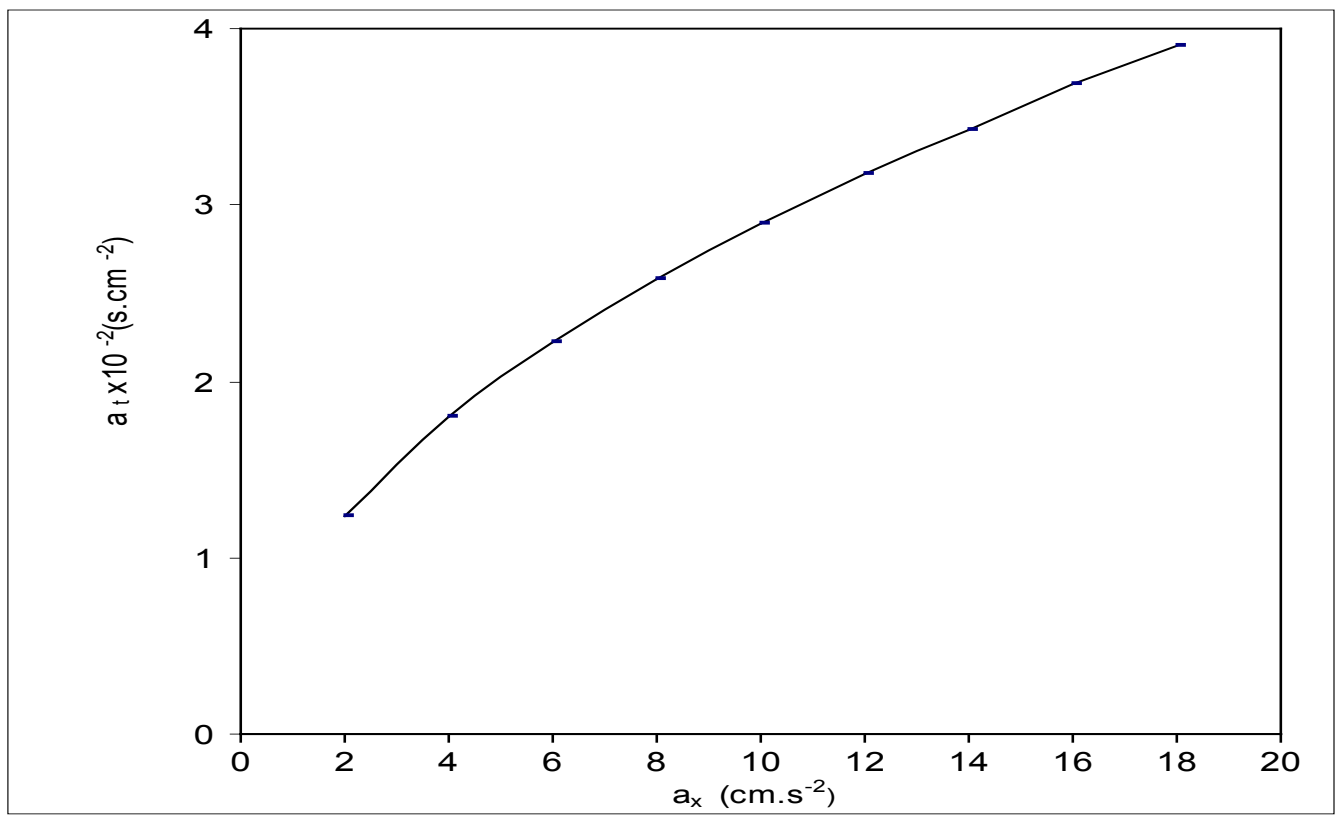

Figure 2 : time acceleration versus dimension acceleration of postulated values 


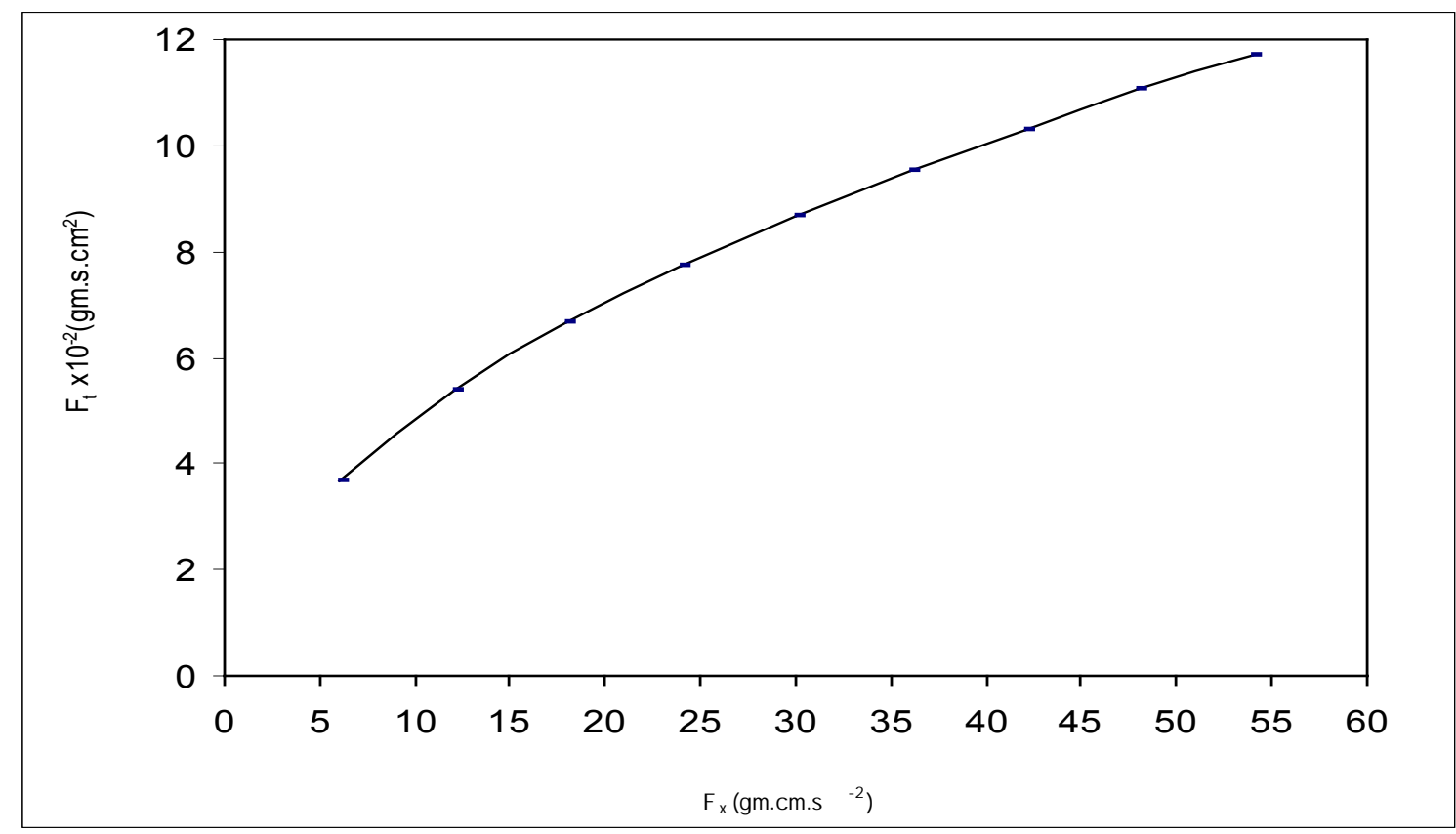

Figure 3 : time force versus dimension force of postulated values

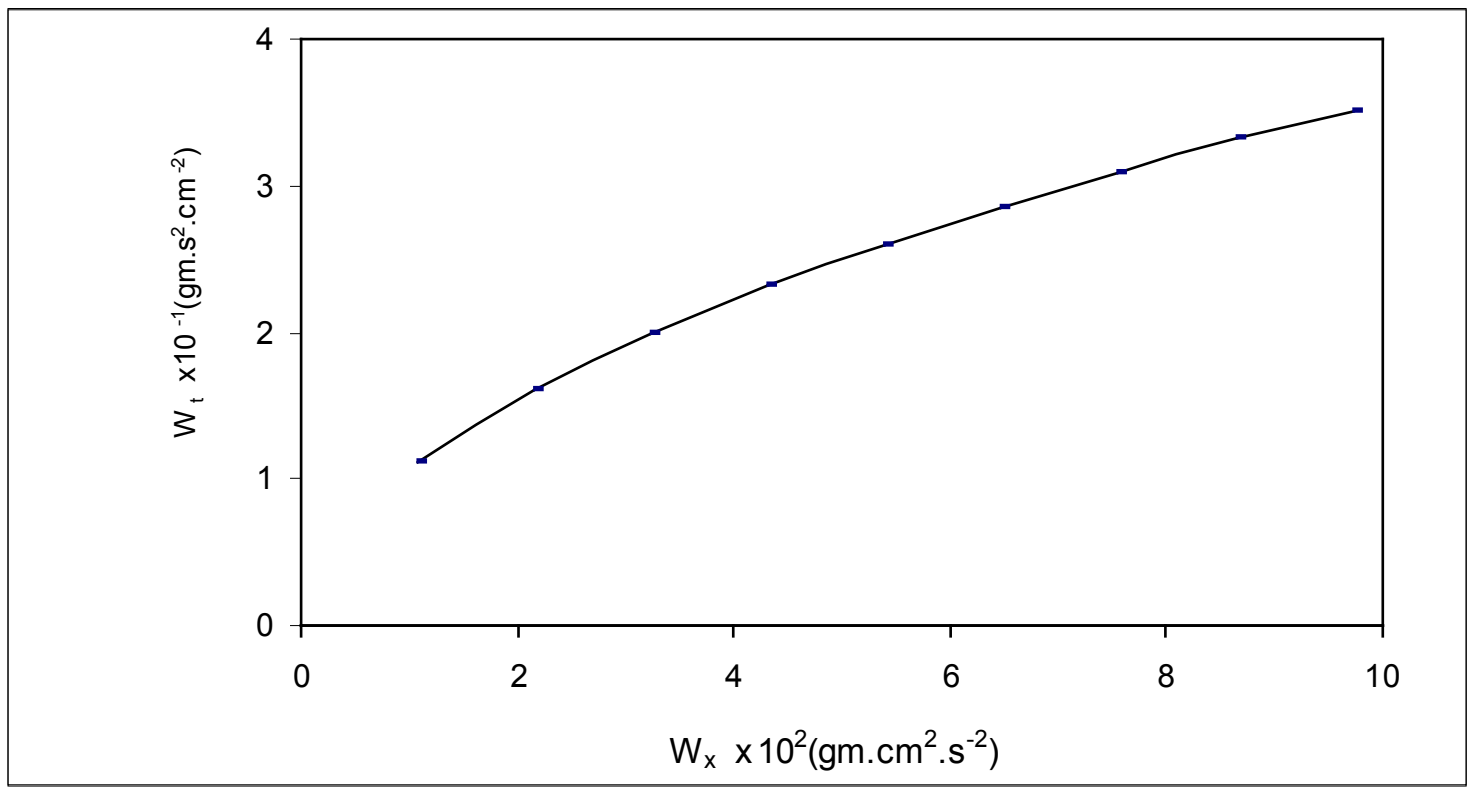

Figure 4 : time work versus dimension work of postulated values

Figure 5 referees to the inversely relationship between the dimension resistivity and its reciprocal(conductivity) of range three types of materials (conductors, semiconductors, and insulators)[17] . The reciprocal relation is valid to find the numerical values of each other. The concept of conductance and resistance is coincidence to give values, that any value is adopted at apply the equation in practical parts.

Figure 6 shows the relationship between $\mathrm{E}_{\mathrm{x}}$ and $E_{t}$ that is linear or no slowness is existence in the path of the time electric field, because the path of the field is different of the path of the particle.
Figure 7 shows the relationship between the dimension and time current densities of any postulated values of moving charge in conductor. Also no slowness in time current density. Figure 8 describes the time power and dimension power that represents a curve. 


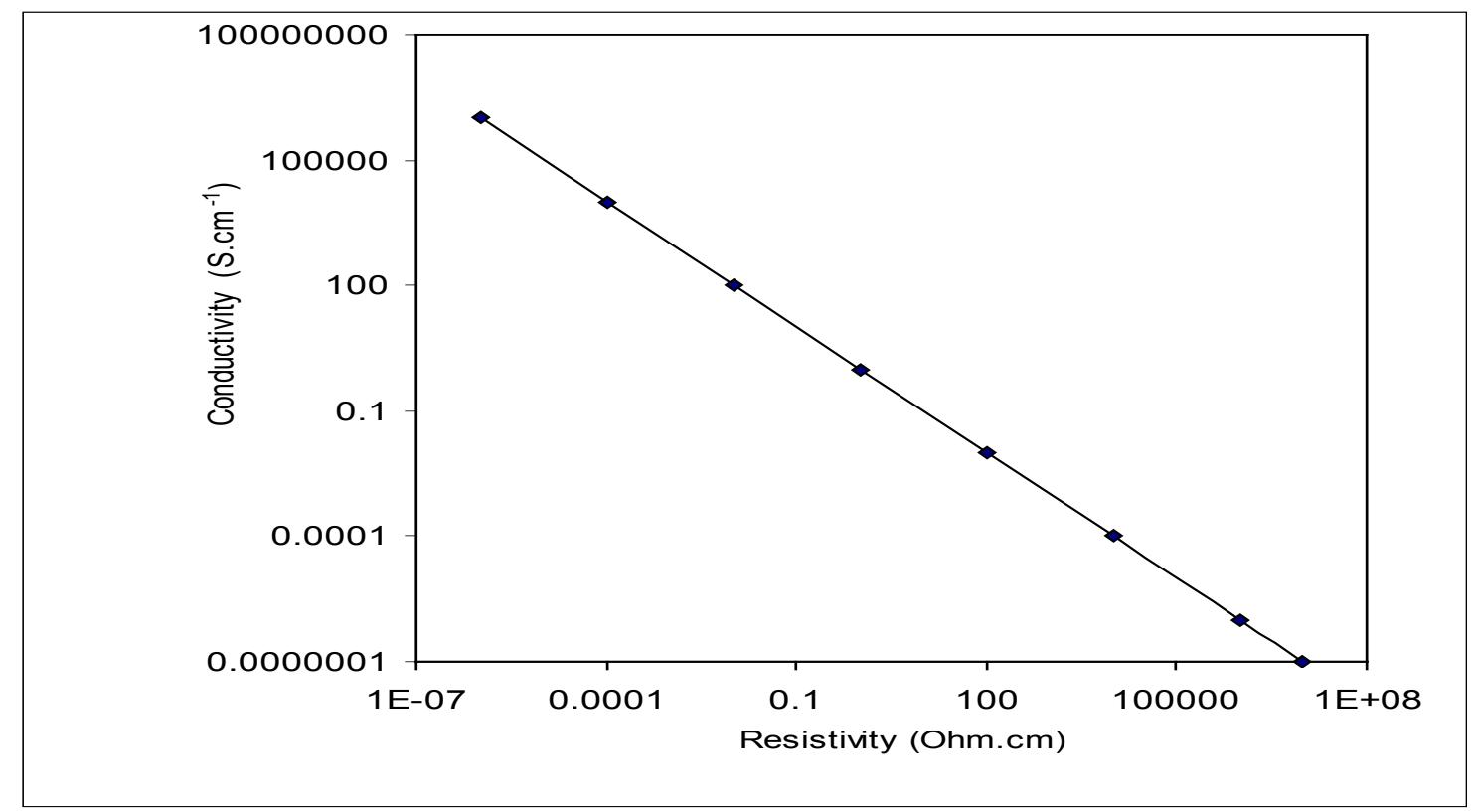

Figure 5 : The inversely relation between the resistivity and its reciprocal of materials

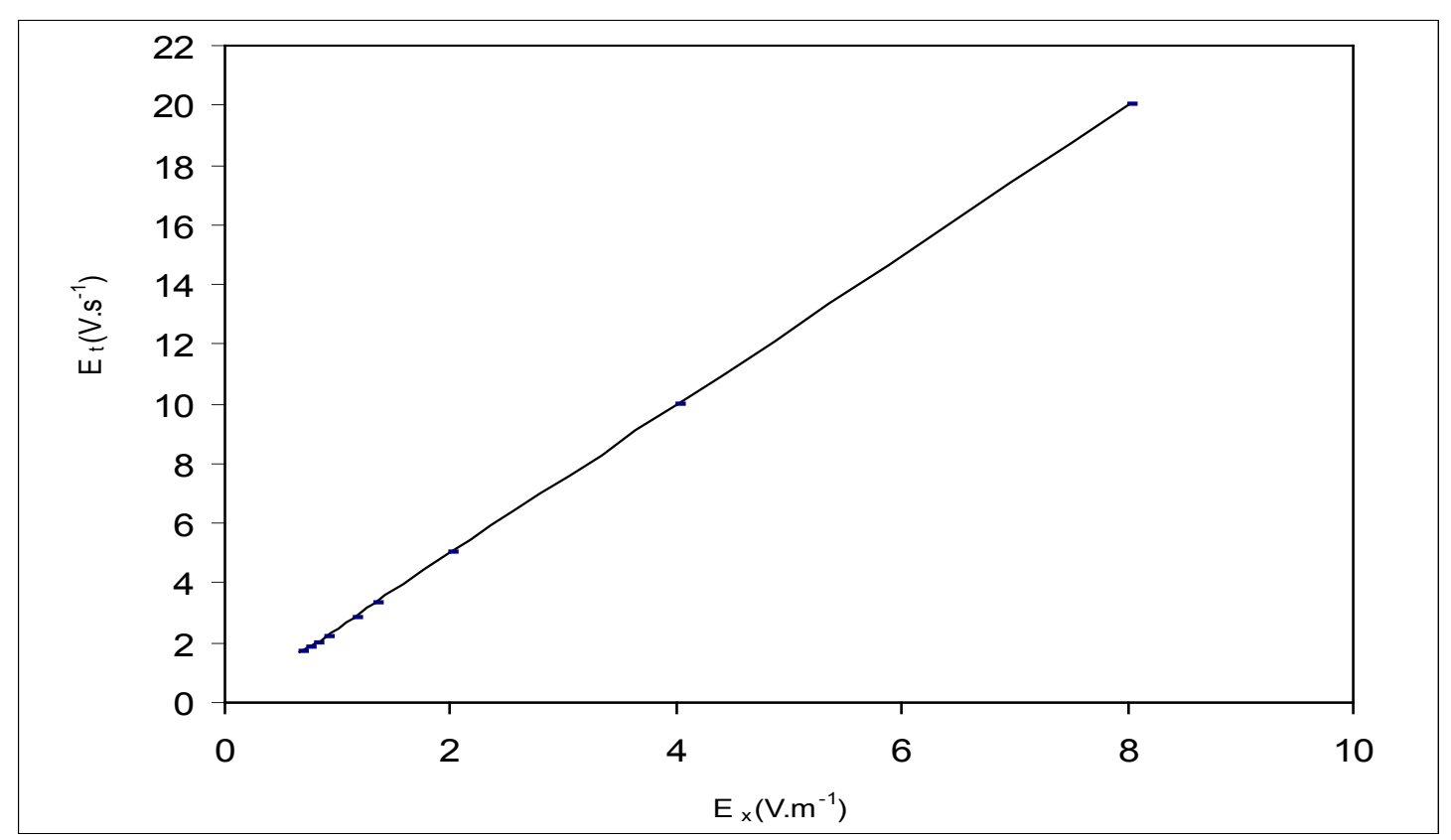

Figure 6: time electric field versus dimension electric field of any postulated values 


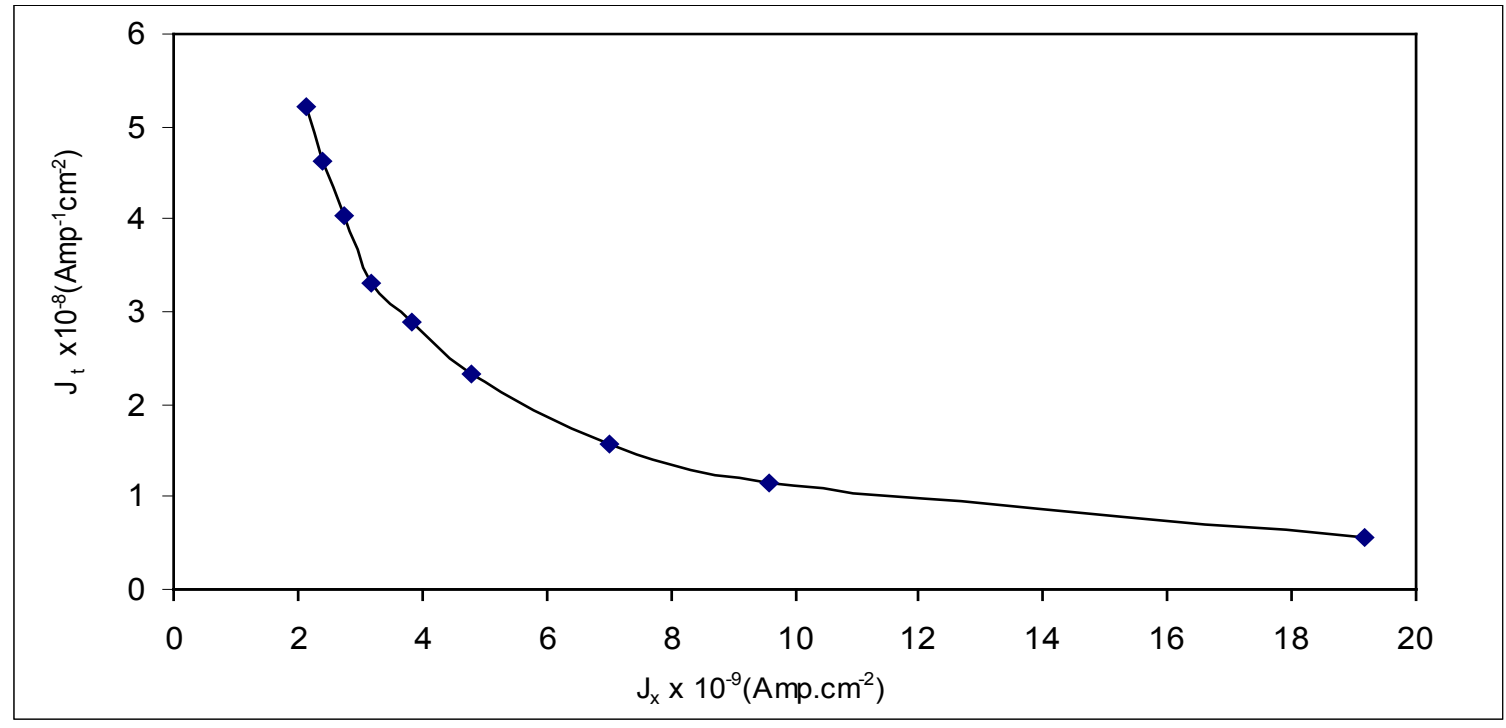

Figure 7 : time current density versus dimension current density of postulated values

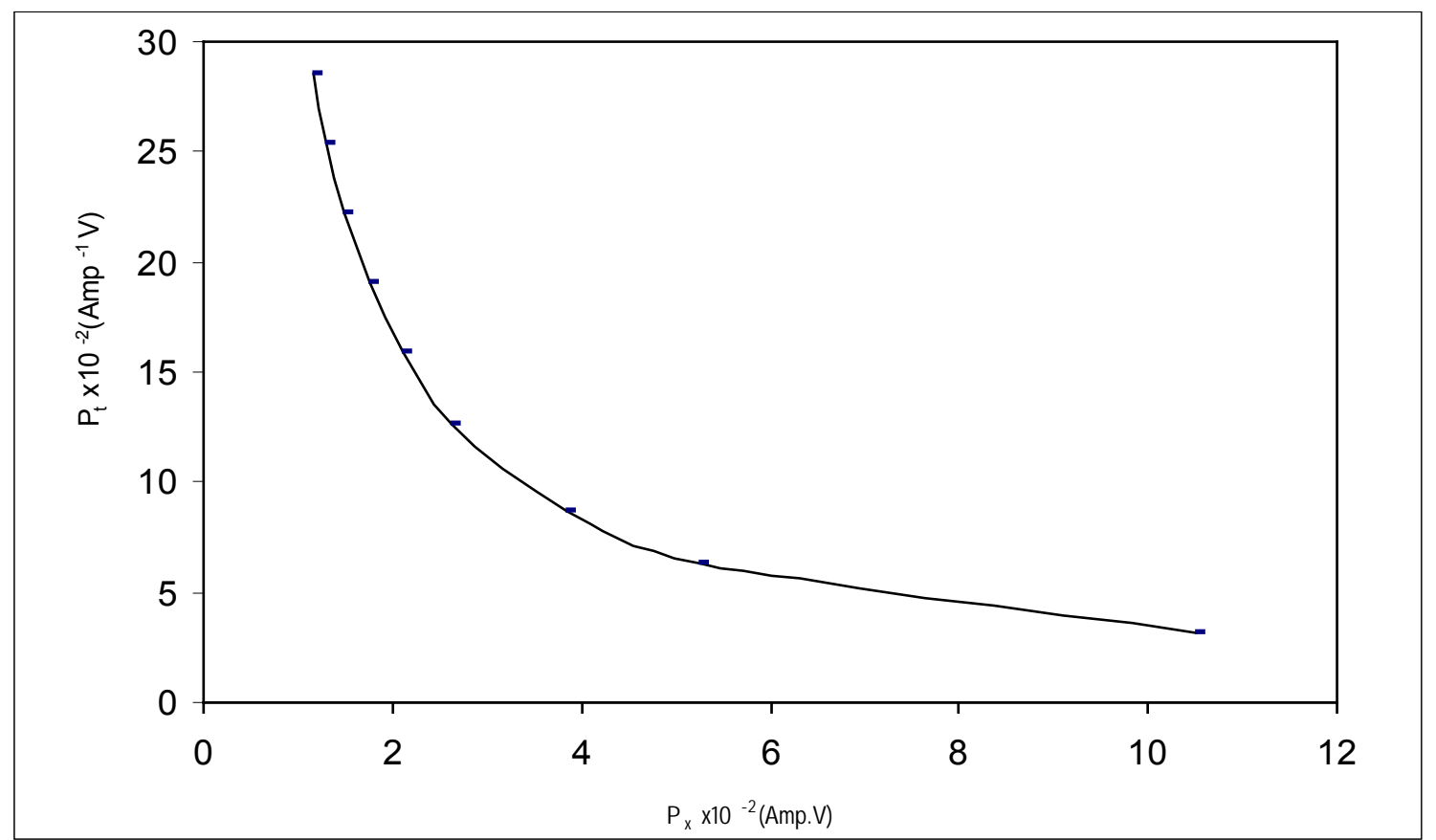

Figure 8 : time power versus dimension power of any postulated values

It is the perfect association of conformalconjugate transformation for a moving particle using a new manner can be applied for another movements such as curvilinear, circular, and rotational motion by using the reciprocal of the quantity. The ability to generalize these new equations is realized by using the same previous steps. Most mechanical instruments and equipment are design to measure the factors of particle or body using dimension equations. In spite of involving timer or any device due to time, it is possible to design equipment due to time equations. The new factors involve more accurate units such as micro and nanometer with large units (e.g. ns $/ \mathrm{m}, \mu \mathrm{s} / \mathrm{cm})$. However the unknown value can be evaluated from one of two types of equations. It must take into account the sign of numerical values for time velocity or time acceleration. The concept of reciprocal velocity or momentum space is important for the classification of electron states in a crystal with respect to time or space. Indeed, the position of an electron cannot be obtained. However, the momentum of an electron can be measured exactly due to uncertainty principle. It is thus clear that we can work with electron states by indexing them in reciprocal space. 
We can produced the reciprocal quantities due to electromagnetic, optical and thermal equations for example : the types of reciprocity due to the focal length of a lens in meters is called power of a lens And the reciprocal of wave number is equal to wavelength. Also the reciprocal of elasticity is the compressibility. The reciprocal of time wave (period)is equal to the frequency. The reciprocal of electric field is equal to the optical non-linear coefficient. That the application of computational values in the new equations for calculation does not contradict with the values in the basic equations, so calculation values give a new concept in a deeper movement. The function of distance and time are the elements that determine the movement of the particle, if we adopted a function of time or a function of distance. There is no difficulty in determining the direction of the movement. So it was noted that an inverted quantity may be independent of time or be based on time and in both cases no contradiction with the concept of the movement of the particle, It is the concept of inverted quantitative directional, whether or numerical might infer new quantities. In electrical dynamics laws, it is observed that the applicability of the concept of new quantity between conductivity and resistivity has given physics concepts for material resistance to the movement of the electron, which we concluded the concept of time current is the reciprocal of dimension current(basically current) that meant the time required for the passage a unit charge. Also the same case apply for time current density by applying any numerical values assumed $m$ the results will be valid in light of the movement of an electron in material.

\section{Conclusions}

1. The reciprocal of dimension velocity has a new formula called time velocity with the same direction. It is a gradient of time for a moving particle. The time acceleration is the Laplacian operator of time, which is a non-vector quantity. The linear and rotational time equations have another new style to evaluate the parameters of moving particle, moreover involving a new concept of a moving particle and design instrument related to time.

2. Many equations of relative time velocity compatible with relative dimension velocity are produced to describe the translational and rotational relative motion of a body due to Galilean and Lorentz transformations.

3. The reciprocal of some electric quantities and its derivatives give a wide knowledge of moving charges related to dimensionally and timely. The values that substituted in the two class laws don't change the numerical values.

4. The values that substituted in the laws of mechanical and electrical quantities either dimensionally or timely contribute to give wide range of knowledge the phenomenon of all factors.

\section{References Références Referencias}

1. Mushfiq Ahmed, J. Sci. Res. 1 (2), 270-274,(2009), Depart. of Phys., Rajshahi, Univ.(6205),Bangladesh.

2. Mushfiq Ahmed, "Discrete and Continuous Representation of the same Motion", Physics Essay, Vol.22 No. 1 March,(2009).

3. MD SHAH ALAM, M. H. AHSAN, and MUSHFIQ AHMED," Mixed Number Lorentz Transformation", J. Natn. Sci. Foundation, Sri Lanka, (2005), 33(1):43 - 49 .

4. Mushfiq Ahmed, International Journal of Applied Physics and Mathmatics, (2012) Vol. 2(5): 336-337 ISSN: 2010-362X.

5. L.A. Rasmussem," Calculation of a Velocity Distribution from Particle Trajectory End Points", Journal of Glaciology, Vol.29 No.102, USA, (1983).

6. Victor Felix de Bottan," Theorem of Movement", Kaplan Place 12, Beersheva 87288,ISDN,(2006).

7. Larry Andrew, Ronald Philips, (Mathematical Techniques for Engineers and Scientists), Publication April, Published by SPIE, the International, Society for Optical Engineering,( 2003).

8. Benjamin Clifford, Queen Mary \& Westfield College (Newton's Principia) M. Sc. Project for Submission 30th.April, (2001).

9. (Foundations of Computational Mathematics), FCM (2002), Minneapolis, 5-14, Classification Problems in Germany, M. Anderson, August (2002).

10. Lim Siow Jin- DXN Institute- Wiseman DXN, 213, Lebuhraya S. A. Halim, 05400,Alor Setar, Kedah, Malaysia, 16 June (2002).

11. Bohren, C. F. and Huffman, D.R. "Absorption and Scattering of Light by Small Particles", Wiley, (1983).

12. F. A. Mustafa," New Formulation of the Reciprocal of Velocity for Particle Motion" Vol.12, No. 3,(2006),J. of Applied and Pure Science of Babylon Univ.

13. F. A. Mustafa, "Uniform Relative Reciprocal Velocity in Lorentz -Einstein Transformations ", British J. of Science, Feb. Vol.4(1),(2012), British Journal ISSN 2047-3745.

14. Alonso- Finn (Fundamental University Physics) Fifth Printing Addison- Wesely-Company Inc. 1980.

15. Dukhin, A.S. and Goetz, P.J. "Ultrasound for characterizing colloids", Elsevier, (2002).

16. James S. Walker, "Physics"(2002), by Prentic Hall,Inc.USA.

17. S. M. Sze and Kwok K. Ng" Physics of Semiconductor Devices", (2007),3rd Edition by Copyright 0 John Wiley \& Sons, Inc. 


\title{
Factor Analysis of the Styrene Polymerization Kinetics Statistical Verification of the Procedure
}

\author{
By V. P. Savel'yanov \\ University of Chemical Technology of Russia, Russian Federation
}

Introduction- Statistical verification of operations used earlier for kinetics experimental data treatment of the styrene suspension polymerization performed on the one of known kinetics model. Internal standard times matrix for different conversions found to have only two statistically significant eigenvalues. Therefore, they may be considerate as plains and easily described to give equation for its dependence from initial condition needed for dimensionless time scale definition. Conversion vectors dependence on the later forms sets of very closely curves. They may be considerate as stochastic realization of the one common curve what follows from its non parametric statistical comparison. Final single curve finds by uniting and sorting operation of its values. That operation gives one plain common curve described by empirical equation with small set of the empirical parameters needed, considerably less than in existing models. Procedure is convenient and robust.

GJSFR-A Classification : FOR Code: 249999p

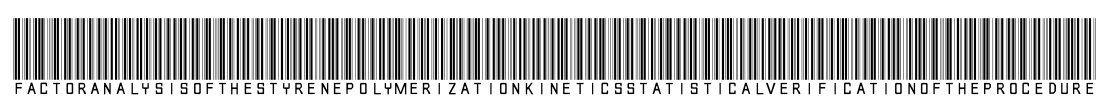

Strictly as per the compliance and regulations of:

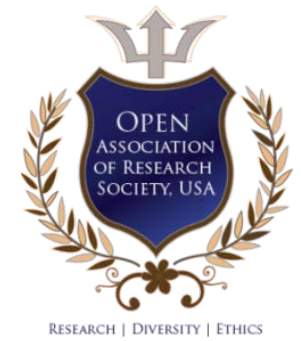

(c) 2015. V. P. Savel'yanov. This is a research/review paper, distributed under the terms of the Creative Commons AttributionNoncommercial 3.0 Unported License http://creativecommons.org/licenses/by-nc/3.0/), permitting all non commercial use, distribution, and reproduction in any medium, provided the original work is properly cited. 


\title{
Factor Analysis of the Styrene Polymerization Kinetics Statistical Verification of the Procedure
}

\author{
V.P. Savel'yanov
}

\section{INTRODUCTION}

$\circlearrowleft$ tatistical verification of operations used earlier for kinetics experimental data treatment of the styrene suspension polymerization performed on the one of known kinetics model. Internal standard times matrix for different conversions found to have only two statistically significant eigenvalues. Therefore, they may be considerate as plains and easily described to give equation for its dependence from initial condition needed for dimensionless time scale definition. Conversion vectors dependence on the later forms sets of very closely curves. They may be considerate as stochastic realization of the one common curve what follows from its non parametric statistical comparison. Final single curve finds by uniting and sorting operation of its values. That operation gives one plain common curve described by empirical equation with small set of the empirical parameters needed, considerably less than in existing models. Procedure is convenient and robust.

We have recently found [1] that the styrene dispersion polymerization kinetics described by simple semi empirical equation with only six empirical constants:

$$
x_{M}=1-0.5\left[\exp (-a \theta)+\exp \left(-b \theta^{c}\right)\right]
$$

where:

$$
\begin{aligned}
& x_{M} \text { - monomer conversion, } \\
& a, b, c \text { - empirical constants, } \\
& \theta \text { - dimensionless time defined as }
\end{aligned}
$$

$$
\theta=\frac{\tau}{\tau_{0.6}}
$$

$\tau$ - astronomical time,

$\tau_{0.6}$ - the internal standard time (IST) defined as the time of $60 \%$ conversion of the monomer.

The dimensionless time found to be the linear function of the initial conditions: temperature $T$, and initial initiator concentration $I_{0}$ with the quite practically acceptable standard deviation $8.123 .10^{-3}$ :

Author: D.Sc. (Chemical Kinetics and Catalysis), Professor, Professor of the Mendeleyev University of Chemical Technology of Russia (MUCTR), Novomoskovsk Division, Druzhby 8, Novomoskovsk, Tula Region, Russia.e-mail: Savelyanovvp@yandex.ru

$$
\ln \tau_{0.6}=A+B T^{-1}+C \ln I_{0} .
$$

These equations we have obtained in the frame of the new procedure, which consist of the a priori experiment design for following factor analysis using. Our experiment space have been created by the quadratic matrix $5 \times 5$ non coincidental combinations of the initial 5 temperatures and 5 initiator concentrations. Experiment results have given us 25 kinetics curves, i.e. monomer conversion dependence on the astronomical time. That way the experiment space created. That space than transformed in the dimensionless form by transition from astronomical time to dimensionless one by formula (2) after Dilman and Polanin [2]. Basic conversion needed chosen equal to 0.6 because at that point gel effect initiates $[1,3]$. Internal standard time matrix have found to be plane in the limits of the experiment accuracy and described by the equation (3). The kinetics curves in the new coordinates $x_{M}-\theta$ found very close one to the other and seems to belong to the only one curve. For finding the later we used the new procedure: uniting them by the placing corresponding arrays one top to the other bottom. The resulting common array values than sorted in ascending order. Because of the large number of experimental points such procedure was performed initially to the 5 initial condition matrix columns giving 5 corresponding united and sorted arrays, and then performing the same operation to all them. Uniting and sorting operation used also to find dimensionless time vector. This way one common array for dependence of the conversion from dimensionless time have derived, which have been excellently seen, corresponds qualitatively to the reaction with the pronounce gel effect described by the equation (1). However, such procedure had two indefinites to proving. The first is indefinites in the basic conversion choosing, and the second - statistically not based uniting and sorting operations with the arrays. These indefinites proving is current communication task.

Verification performed by numerical experiment on the kinetics model close qualitatively to our experimental data, namely - Santos et al [3]. That model bases on the classical radical polymerization scheme in terms of quasi steady state approximation and quadratic active chains termination [4] corrected by introducing initiator affectivity factor (cage effect supposed constant $-f=0.5$ ) and empirical gel effect description. 
The last used exponential multiplier for termination rate constant, containing regression third order equation with linear dependence its coefficients on temperature. That multiplier contains six purely empirical constants.

Kinetics curves produced by numerical integration that model at the initial condition identical to matrix in the real investigation [1], i.e. temperatures 78, $81,84,87$, and $91 \mathrm{C}$, and initiator concentrations 0.021 , $0.033,0.045,0.058$, and $0.070 \mathrm{~mol} / \mathrm{l}$. Ten equidistant points from interval $\{0,1\}$ chosen to form the points of the simulated "kinetics curves". The 25 kinetics curves was the result, which formed the experimental space in time and initial conditions coordinates. Space cross section at conversions $0.2,0.3,0.4,0.5,0.6$ and 0.8 produced corresponding internal standard time matrixes. All they found to have rank 5 . Factor analysis [5] demand their centering and normalization, but we ignored these operations. The first of them needed for matrix rotation and the later standardized values by the variances that may differ in time. We considerate all curves as the section at the same conversion what did the later operation excessive. The matrix centering we applied only for excluding large number in calculating because rotation will lead to emergence of new variable - linear combination of the initial, and all description serious complication.

Results of the matrixes eigenvalues calculation given in the Table 1, from which seen that all them have only two first significant values. Information content in them practically not differs from 1 . To these eigenvalues corresponds two eigenvectors which defines space dimension created by matrix. Therefore, cross sections at any conversion may be considerate as planes, provided that experimental error taken into consideration. Fig. 1 demonstrates that visually.

Table 1 : Centered IST matrixes eigenvalues* at various monomer conversions

\begin{tabular}{|c|c|c|c|c|c|c|}
\hline & \multicolumn{7}{|c|}{ Basic monomer conversion } \\
\cline { 2 - 7 } & 0.2 & 0.3 & 0.4 & 0.5 & 0.6 & 0.8 \\
\hline Eigenvalues & 2.937 & 2.854 & 2.77 & 2.688 & 2.623 & 2.623 \\
$\lambda$ & 1.25 & 1.3 & 1.347 & 1.394 & 1.43 & 1.43 \\
& $1.381 \times 10^{-5}$ & $2.951 \times 10^{-6}$ & $2.815 \times 10^{-6}$ & $4.516 \times 10^{-6}$ & $1.892 \times 10^{-6}$ & $1.892 \times 10^{-6}$ \\
& $2.533 \times 10^{-7}$ & $5.806 \times 10^{-7}$ & $6.209 \times 10^{-7}$ & $7.911 \times 10^{-7}$ & $8.067 \times 10^{-7}$ & $8.067 \times 10^{-7}$ \\
& $1.179 \times 10^{-10}$ & $1.355 \times 10^{-6}$ & $1.198 \times 10^{-8}$ & $2.624 \times 10^{-10}$ & $7.615 \times 10^{-8}$ & $7.615 \times 10^{-8}$ \\
\hline Matrix rank & 5 & 5 & 5 & 5 & 5 & 5 \\
\hline $\mathrm{R}^{\text {** }}$ & 1 & 1 & 1 & 1 & 1 & 1 \\
\hline
\end{tabular}

)$^{*}$ centering performed referring to the central matrix value instead of the mean one.

)$^{* *}$ the information fraction of the first two values: $\left(\lambda_{1}+\lambda_{2}\right) / \Sigma \lambda$.

The planes only slightly curved, and curving induces by initiator. This is seen from correlation coefficients for matrix columns and rows: for temperature ones they all strictly are 1 , and only close to 1 for initiator concentration (ca. 0.985).

These results mean that equation (3) may be applied for the IST dependence from initial condition full description. Table 2 confirms that conclusion. It must be noted that in that case the better correlation obtained with the modified form of this equation (3) where initial initiator concentration used instead of its logarithm, i.e.

$$
\ln \tau_{x}=A+B T^{-1}+C I_{0} .
$$

Table 2 : Regression coefficients of the equation (4)

\begin{tabular}{|c|c|c|c|c|c|c|}
\hline \multirow{2}{*}{ Coefficient } & \multicolumn{5}{|c|}{ Basic monomer conversion } \\
\cline { 2 - 7 } & 0.2 & 0.3 & 0.4 & 0.5 & 0.6 & 0.8 \\
\hline A & 25.755 & 26.387 & 27.589 & 28.707 & 28.831 & 29.778 \\
B & -6548 & -6667 & -7015 & -7356 & -7353 & -7.654 \\
C & 19.386 & 19.073 & 18.743 & 18.406 & 18.121 & 17.818 \\
\hline Standard & 0.045 & 0.045 & 0.047 & 0.049 & 0.050 & 0.053 \\
deviation & & & & & & \\
\hline
\end{tabular}

One may see from table 2 that equation (4) describes planes rather well but regression coefficients vary with the basic conversion. $A$ and $B$ increase while the $C$ diminishes noticeably. One may see also increasing of the standard deviation. Thus, in the whole, such description seems to be acceptable - especially for practical purposes. All six matrixes shown on the Fig. 1 form visually coplanar plains - that seen visually very well. 


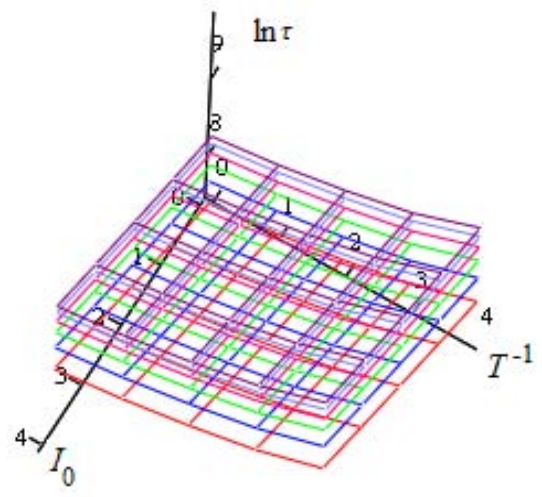

Figure 1: IST dependence on initial conditions at monomer conversions: $0.2,0.3,0.4,0.5,0.6,0.8$ (down to up)

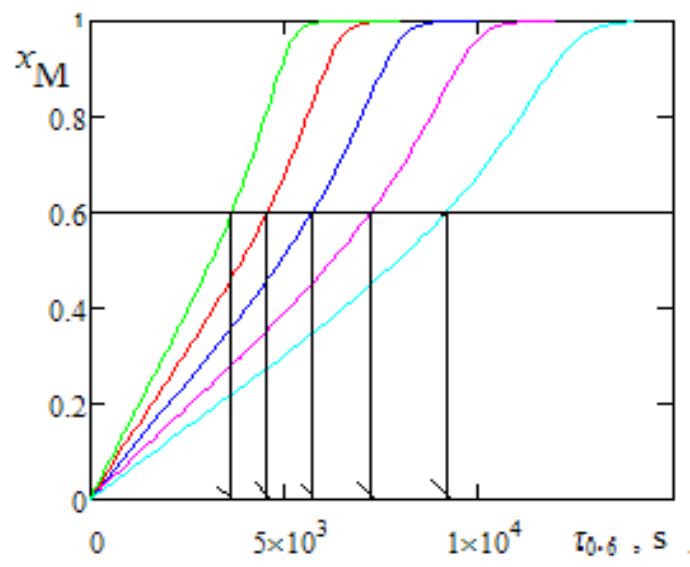

Results of the uniting and sorting operation application to the vectors artificially produced (kinetics curves) shown on the Fig 2. The common curve seen very well. Diverging observed at high conversions (more than 0.95 - see Fig.3) and has order approximately 0.01-0.02 in the maximum. This diverging depends on the basic conversion chosen for dimensionless time determination and diminishing the later increase it noticeably. 60\% conversion have been chosen in [1] rather well. From Fig. 3 one may see also that uniting and sorting operation are equivalent, in some sense, to meaning all curves.

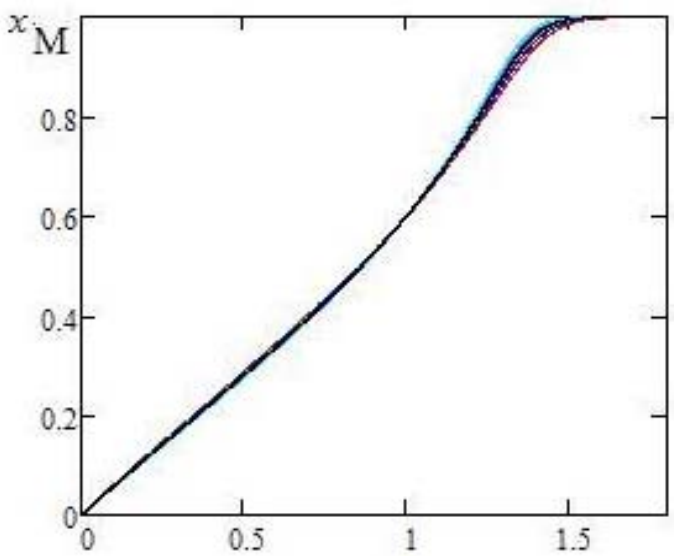

Figure 2 : Kinetics curves, and times of the $60 \%$ conversion determination (shown by arrows) for temperature series $(78,81,84,87$ and $90 \mathrm{C})$ at the initiator concentration $0.07 \mathrm{~mol} / \mathrm{l}-$ left. Kinetics curves in the dimensionless time scale and operation result of its uniting and sorting (black curve) - right

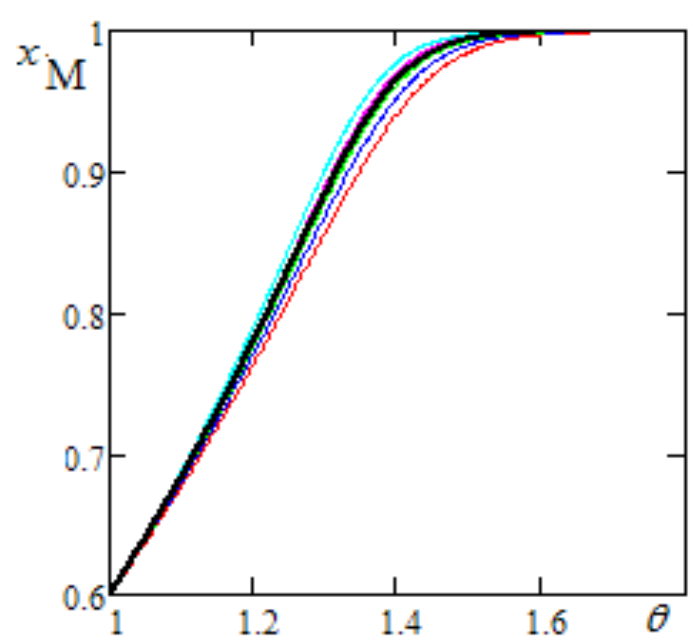

Figure 3: Conversion vs. dimensionless time curves from Fig. 2 at high conversions. There is no full coinciding. Fat black curve is the result of uniting and sorting operation on all five individual curves

Operation of experimental vectors uniting and sorting needs special attention because not only its role in the resulting equation obtaining but its amazing effectiveness also. One may see that if compares initial experimental curves and final one in [1]. Just that gives us base consider it earlier justified a posteriori [6]. We prove that operations on the model in two ways: in the reproducibility "runs", and in one series treating. Fig.4 represents results of the simulation data treatment with "experimental error". The last modeled by addition to calculated values of conversion normal distributed random variable with null mean and variance 0.04 (standard deviation 0.2!). Initial model kinetics curve reproduces quite well, providing that dimensionless time vectors treat by the same procedures uniting and sorting. Fig. 4 demonstrates how these operations works for serial data. Initial curves do not indentify even its form which seen well on the united and sorted vector. Fig. 5 demonstrates that procedure for reproducibility five runs. Common vector curve reproduced model curve quite well. 

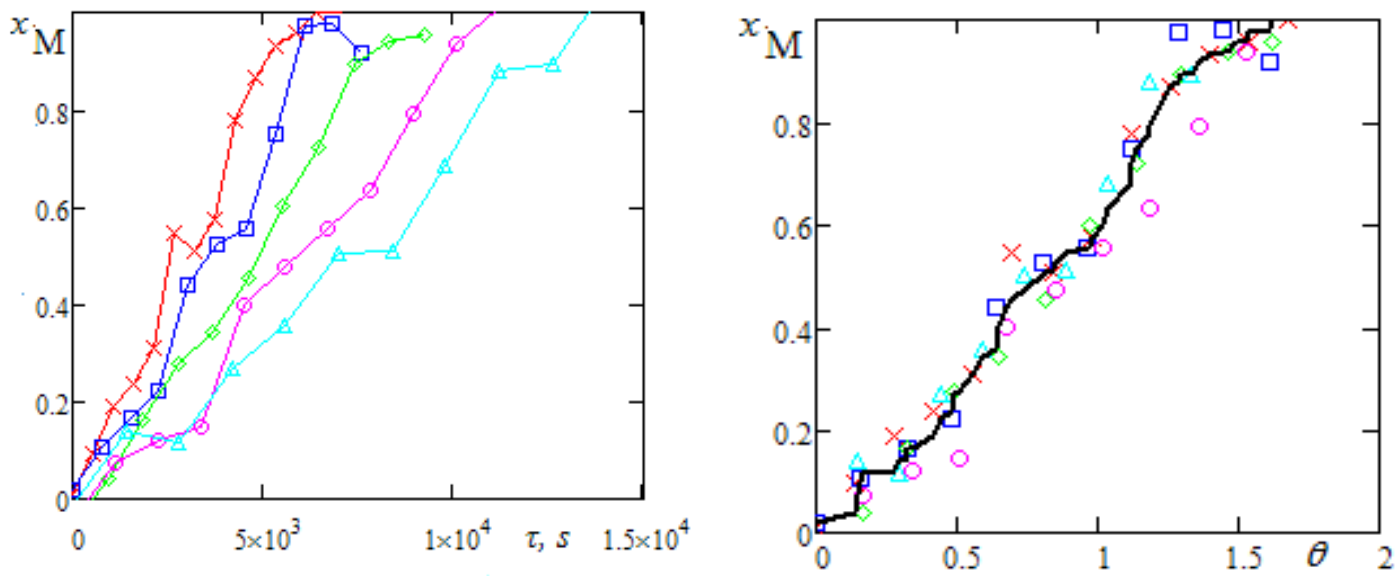

Figure 4 : Simulated kinetics curves at initiator concentration $0.070 \mathrm{~mol} / \mathrm{l}$, and different temperatures. Standard deviation equals 0.2. Five "experimental" curves - on the left. United and sorted common vector - on the right

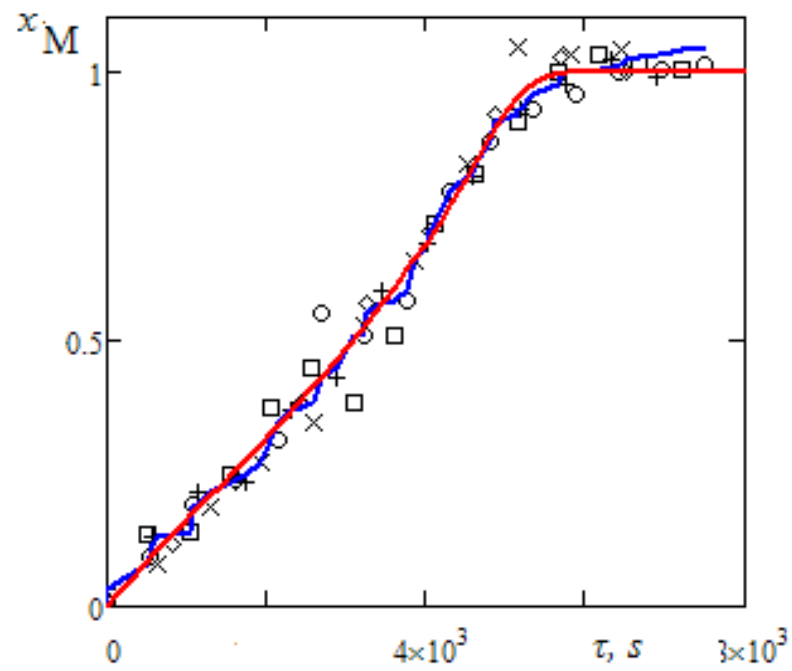

Figure 5 : Stack and sort operations illustration for simulated reproducibility runs. Points are individual five "runs" results with standard deviation 0.2 , blue line is result of uniting and sorting operations, and red line is the model. Unreal conversions, larger than 1.0, are included intentionally for procedure demonstration

The procedure used bases on the postulate that all experimental curves are stochastic realization of only one common curve. One may see that from Fig. 3 and this statement have to prove. Small experimental points we have exclude using of any parametrical criterion and nonparametric one from Wilcoxson (Mann-Witney) was used [7]. For proving, we take border curves having maximum differences, namely at $78 \mathrm{C}$ and $90 \mathrm{C}$ (initiator concentration $0 / 07 \mathrm{~mol} / \mathrm{l}$ ). The procedure [7] include generating one common vector from two vectors compared, its sorting in ascending order, and ranging its values by natural number sequence. Then rank sum calculated for the initial vectors for criterion values calculation. The smaller one compared with critical one from statistical table, which must be smaller than calculated in the case of the difference insignificancy of the two vectors compared. The following example also gave vectors uniting and ranging operation illustration.

In our case, vectors compared are (two temperatures at initiator concentration $0.07 \mathrm{~mol} / \mathrm{l}$ ):

$$
\begin{aligned}
& x m 51(0,0.105,0.206,0.306,0.409,0.521,0.650,0.804,0.955,0.997,1.000) \\
& x m 55(0,0.086,0.169,0.253,0.340,0.433,0.537,0.661,0.817,0.967,0.999)
\end{aligned}
$$

United and sorted vector is: 
Xm5 (0,0,0.086, 0.105,0.169, 0.206, 0.253,0.306, 0.340, 0.409,0,433, 0.521,0.537,0.650,0.661, 0.804, 0.817, $0.955,0.967,0.997,0.999,1.000)$

and corresponding rank vector is:

$$
\operatorname{RXm5}(1,1,2,3 \ldots 21)
$$

Initial vectors ranks and their rank sum:

$$
\begin{array}{ll}
\text { Rxm51 }(1,3,5,7,9,11,13,15,17,19,21) & \text { SRxm51 }=121 \\
\operatorname{Rxm55}(1,2,4,6,8,10,12,14,16,18,20) & \text { SRxm55 }=131
\end{array}
$$

Criteria calculated in such way [6]

$$
U 5 i=\sum R x m 5 i-\frac{N i(N i+1)}{2}
$$

where $\mathrm{Ni}$ is $\mathrm{i}$-vector length. The smaller criterion calculated is 55 . Critical criterion value is 25 on the $1 \%$ significance level [6]. All other calculated criteria are more than that value. Thus, all five vectors are indistinguishable statistically and may considerate as stochastic realization of the one common vector. This conclusion justifies procedure described above - uniting all five vectors in common one and sorting its values in ascending order.

\section{Conclusion}

The procedure developed basis on consciously experiment design for subsequent factor analysis methods using. It results in the substantial simplification of the kinetics description for styrene polymerization in full interval of monomer conversion. The experiment space found to need for full description only six empirical parameters when literature models include 13 (and more!) such parameters. The new operations in the data treatment, i.e. internal time standard introduction for time dimentionlessing and subsequent uniting and sorting conversion and time vectors values leads to one common two-dimensional kinetics curve. Its empirical description is pure technical task. All these operation are statistically valid as numerical experiment on the one of kinetics model demonstrated even without stochastic experimental error. The initial kinetics curves reproduce well. The procedure seems to be robust, simple and convenient - especially with contemporary PC program using. The single restriction is that only ascending (or descending) experimental curves may be treated.

The procedure includes some interesting features needed feather investigation. The vectors uniting and ranging operation high efficiency remain incomprehensible. Perhaps, there is united and sorted conversion and dimensionless time vectors autocorrelation because of its determination from the same experimental curves set. Resulting two dimensional curve empirical descriptions definitely demands two different functions sum using. It is possible that in reality we have two different polymerization mechanisms - diffusion complicated and classical in macromolecular globules and monomer phases, existing really up to glass effect point. It is seems interesting to note that the second term in equation (1) has form typical for process of crystallization. Finally, it is possible that procedure will be useful for searching empirical dependencies from experimental data [7] - not only for kinetics time series.

\section{References Références Referencias}

1. Vent, D.P., Savelyanov, V.P., Lopatin, A.G., and Safin, M.D. Applied kinetics of styrene dispersion polymerization. Theoretical Foundation of Chemical Engineering.2014.Vol.48.No.3.pp.332-336.

2. Dil'man, V.V., Polanin A.D. Methods of the model equations and analogies in chemical technology. Moscow. Chemistry. 1988 (In Russian: Dil'man, V.V., Polanin A.D. Metody model'nykh uravneniy i analogiy $v$ khimicheskoy tekhnologii Moskva. Khimiya).

3. Santos, J.C., Lopez, C.N, Reis, M.M., Giudici, R., Sayer, C., Machado, R.F.H., and Araujo P.H.H. Comparison of techniques for determination of conversion during suspension polymerization reactions. Braz. J. Chem. Eng. 2008. vol.25. p.399.

4. Savelyanov V.P. Basics of polymer chemical technology. Moscow. IKC Academbook, 2007 (In Russian: Obshchaya khimicheskaya tekhnologiya polimerov. Moskva. IKC Akademrniga. 2007).

5. Computer aids to chemistry/ Verner, J. and Charon F. Chichester. Ellis Horwood Ltd. 1986.

6. Savel'yanov, V.P. On the operation of vectors merging and sorting in its sets. Tula State University Communication. Natural sciences. 2014. V. 1. Part 2. p. 156-161 (In russian: Savel'yanov, V.P. Ob operatsii sliyaniya i sortirovki vektorov $v$ ikh seriyakh. Izvestiya Tulskogo Gosudarstvennogo Universiteta (TulGU). Estestvennyye nauki. 2014. Vip.1.Chast 2.S.156-161).

7. Lakin G.F. Biometrics. Moscow. High School. 1990. P. 130-131. (In Russian: Biometria. Vysshaya Shkola. Moskva.1990).

8. Vapnik V.N. Estimation of dependencies based on empirical data. Springer. N.Y. 1982 


\title{
Is Spacetime Fractal and Quantum Coherent in the Golden Mean?
}

\author{
By Mae-Wan Ho, Mohamed el Naschie \& Giuseppe Vitiello
}

Abstract- We consider the fabric of spacetime from a wide perspective: from mathematics, quantum physics, far from equilibrium thermodynamics, biology and neurobiology. It appears likely that spacetime is fractal and quantum coherent in the golden mean. Mathematically, our fractal universe is non-differentiable and discontinuous, yet dense in the infinite dimensional spacetime. Physically, it appears to be a quantum coherent universe consisting of an infinite diversity of autonomous agents all participating in co-creating organic, fractal spacetime by their multitudinous coupled cycles of activities. Biologically, this fractal coherent spacetime is also the fabric of conscious awareness mirrored in the quantum coherent golden mean brain states.

Keywords: whitehead's philosophy, discontinuous nondifferentiable spacetime, fractals, coupled activity cycles, deterministic chaos, quantum coherence and fractals, golden mean.

GJSFR-A Classification : FOR Code: 020699, 020109

Strictly as per the compliance and regulations of:

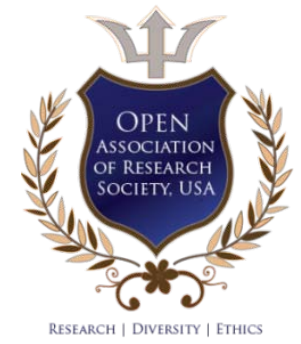

(C) 2015. Mae-Wan Ho, Mohamed el Naschie \& Giuseppe Vitiello. This is a research/review paper, distributed under the terms of the Creative Commons Attribution-Noncommercial 3.0 Unported License http://creativecommons.org/licenses/by-nc/3.0/), permitting all non commercial use, distribution, and reproduction in any medium, provided the original work is properly cited. 


\title{
Is Spacetime Fractal and Quantum Coherent in the Golden Mean?
}

\author{
Mae-Wan $\mathrm{Ho}^{\alpha}$, Mohamed el Naschie ${ }^{\sigma}$ \& Giuseppe Vitiello ${ }^{\rho}$
}

\begin{abstract}
We consider the fabric of spacetime from a wide perspective: from mathematics, quantum physics, far from equilibrium thermodynamics, biology and neurobiology. It appears likely that spacetime is fractal and quantum coherent in the golden mean. Mathematically, our fractal universe is non-differentiable and discontinuous, yet dense in the infinite dimensional spacetime. Physically, it appears to be a quantum coherent universe consisting of an infinite diversity of autonomous agents all participating in co-creating organic, fractal spacetime by their multitudinous coupled cycles of activities. Biologically, this fractal coherent spacetime is also the fabric of conscious awareness mirrored in the quantum coherent golden mean brain states.
\end{abstract}

Keywords: Whitehead's philosophy, discontinuous nondifferentiable spacetime, fractals, coupled activity cycles, deterministic chaos, quantum coherence and fractals, golden mean.

\section{InTRODUCTION}

$\mathrm{s}$ s spacetime fractal and quantum coherent in the golden mean? This is too deep and fundamental a question to be answered definitively on the basis of our present knowledge. However, one could try to get a good idea on the properties of spacetime in a number of mathematical and physical features emerging from research activities in many fields, including mathematics, quantum physics, far from equilibrium thermodynamics, biology and neurobiology, which also hark back to some basic problems in philosophy. Our attempt in this paper is to bring together the relevant observations and findings without claiming to be complete, that support our speculation concerning the basic "fabric" of spacetime. In so doing, we connect the ubiquitous recurrence of the golden mean and fractal self-similarity in microscopic and macroscopic phenomena with the coherent state dynamics at the quantum level and effects at the macroscopic level.

We start with the nature of the spacetime as perceived by Alfred North Whitehead (1861-1947) and others.

\section{il. Real Processes do not Happen at Points in a Spacetime Continuum}

As one of us (MWH) has commented [1], Whitehead lived through an exciting era in Western

\footnotetext{
Author a: Institute of Science in Society, Edge Institute International, London UK and Italy.e-mail: m.w.ho@i-sis.org.uk.

Authoro: Alexandria University, Egypt.

Author p: University of Salerno, Edge Institute International, London UK and Italy.
}

science when the fabric of physical reality - Newton's flat, smooth, and static absolute spacetime - was being thoroughly ruffled by Albert Einstein's theories of special and general relativity and by quantum mechanics. The modern observer no longer views nature from the outside, being irreducibly quantum-entangled with the known, possibly with all entities in the entire universe.

These surprising lessons from nature became the basis of Whitehead's perennial philosophy (a cosmogony) [2], ushering in a new age of the organism that inspired generations of scientists. He saw the universe as a super-organism encompassing organisms on every scale from galaxies to elementary particles, and argued it is only possible to know and understand nature both as an organism and with the sensitivity of an organism.

Most important, though least understood was his rejection of the mechanical laws of classical physics and differential calculus for their failure to describe real processes. Not only do they leave out of account the allimportant knowing experiencing organism, real processes occur in intervals of time associated with volumes of space. Absolutely nothing can happen at a point in an instant.

Rather than a smooth, infinitely divisible continuum, spacetime is more likely discrete and discontinuous, and hence non-differentiable, as quantum physics has already discovered at the smallest scale. Unfortunately, mathematics had lagged behind physics. Both relativity theory and quantum theory inherited the predominant mathematics of classical mechanics.

Roger Penrose's The Road to Reality, A Complete Guide to the Laws of The Universe [3] charts the heroic and ingenious efforts of mathematical physicists to grasp hold of post Newtonian universe, which in the end they failed to do. The dream of uniting the two great theories of quantum physics and general relativity has remained unfulfilled; not least because these two modern theories are both based on the foundation of classical physics: a differentiable, continuous, spacetime manifold.

The issue of continuity versus discontinuity of spacetime did not originate in Newtonian mechanics. It can be traced back to ancient Greek philosophy, especially in Zeno's paradox of Achilles and the tortoise [4] (see Appendix 1). It is generally thought that Newton and Leibniz had both resolved Zeno's paradox with 
differential calculus, by inventing infinitesimal space and time intervals. Whitehead [2] and Henri Bergson [5] were among those who would not have accepted this 'resolution'.

Zeno's paradox was about the impossibility of motion as represented by an infinite sequence of static configurations of matter at points in time. Whitehead said in his Concept of Nature ([6] p. 15): "There is no holding nature still and looking at it." The absolute, infinitely divisible time and space of Newtonian physics are both abstractions from ever owing events of nature. He concurred with Bergson [5] in using the concept of 'duration'([6], p. 53) for an interval of time experienced by the knower as a simultaneity encompassing 'a complex of partial events'. We shall show later that 'duration' and 'simultaneity' can be given very specific meanings in terms of characteristic time of processes and coherence time.

\section{ili. Mathematics of Discontinuity}

The Cantor set, discovered by Georg Cantor in 1883 [7], is fundamental for discontinuous mathematics. Its apparently paradoxical nature is that it is infinitely sub-divisible, but is completely discontinuous and nowhere dense. It is also a fractal with self-similar patterns on every scale [8].

The mathematics of non-differentiable and discontinuous spaces is among the most significant discoveries/inventions beginning with Cantor in the late $19^{\text {th }}$ century, though it did not really take off until well into the $20^{\text {th }}$ century, reaching its peak in the science and mathematics of complexity associated especially with Benoit Mandelbrot's fractals [9] and Edward Lorenz' deterministic chaos [10],[11].

We provide informal definitions of some mathematical terms that will be used in this paper in Appendix 2 (from [1]); some, like deterministic chaos, have no generally agreed definition.

\section{Continuous Non-Differentiable Fractal Spacetime}

Garnet Ord was the first to propose a fractal spacetime and to coin the term for it [12]. His starting point was Richard Feynman's observation [13] that when they are looked at on a sufficiently fine scale, the the paths of quantum mechanical particles are nondifferentiable curves rather than straight lines. Moreover, relativistic interaction with particles at sufficiently high energies produces non-conserved particle numbers. These and other anomalies have encouraged quantum mechanics to abandon the concept of a point particle and its trajectory in favour of wavepackets or field excitations. Feynmans formulation in terms of path integrals was an exception. In the same spirit, Ord set out to construct a continuous trajectory in spacetime that exhibits features analogous to those in relativistic quantum mechanics. He came up with a fractal trajectory exemplified by a Peano-Moore curve (Figure 1). It is plane-filling with a fractal (Hausdorff) dimension of 2 instead of the classical linear path that has a dimension of 1 .

Ord showed that, among other things, such a fractal trajectory exhibits both an uncertainty principle and a de Broglie relation. On a microscopic scale, the presence of fractal time is interpreted in terms of the appearance of particle-antiparticle pairs when observation energies are of the order of $m c^{2}$. On a macroscopic scale greater than the fractal wavelength, the free 'fractalons' appear to move on a classical onedimensional trajectory.

A more elaborate scale-relativity theory of fractal spacetime was proposed by Laurent Nottale [14], who was motivated by "the failure of a large number of attempts to understand the quantum behavior in terms of standard differentiable geometry" to look for a possible "quantum geometry", i.e., fractals [15], (pp.45). His theory recovers quantum mechanics as mechanics on a nondifferentiable spacetime, and the Schrödinger equation is demonstrated as a geodesic equation.

Ord and Nottale have both proposed fractal spacetimes that are continuous and nondifferentiable.

A more radical cosmology proposed by one of us (MeN) is a fractal spacetime that is based on the golden mean and is neither continuous nor differentiable (see below). It is closest to our intuitive notion of organic (as opposed to mechanical) spacetime, and more intimately connected to our emerging understanding of biological spacetime [1].

(a)

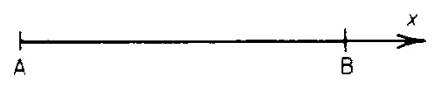

(b)
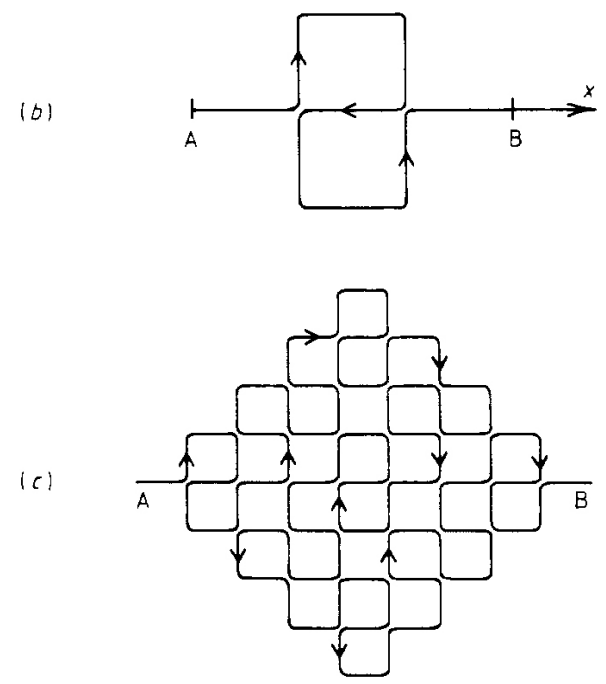

Figure 1: A particle's fractal trajectory at increasing resolution, at scale (a): $s=\lambda,(\mathrm{b}): s=\lambda / 3,(\mathrm{c}): s=\lambda / 9$.

(from [13]) 


\section{V. $\mathcal{E}^{\infty}$ Spacetime And Our 4-Dimensional UNIVERSE}

MeN's work was first reviewed by MWH [1], who provided a simplified account that we recapitulate here.

$\mathcal{E}^{\infty}$ (E-infinity) is an infinite dimensional fractal spacetime. Yet its Hausdorff dimension is $4.236067977 \ldots$ This means that at ordinary scales, it looks and feels 4 dimensional (three of space and one of time), with the remaining dimensions 'compacted' in the remaining 0.236067977 "fuzzy tail" [16]. One imagine such a universe as a four dimensional hypercube with further four dimensional hypercubes nested inside like Russian dolls [17] (see figure 2). The exact Hausdorff dimension of the infinite dimensional hypercube is $4+\phi^{3}$, where $\phi=(\sqrt{5}-1) / 2$, the golden mean. The dimension $4+\phi^{3}$ can be expressed as the following self-similar continued fraction which converges to precisely $4+\phi^{3}$ :

$$
4+\phi^{3}=4+\frac{1}{4+\frac{1}{4+\frac{1}{4+\frac{1}{4+\ldots}}}}
$$

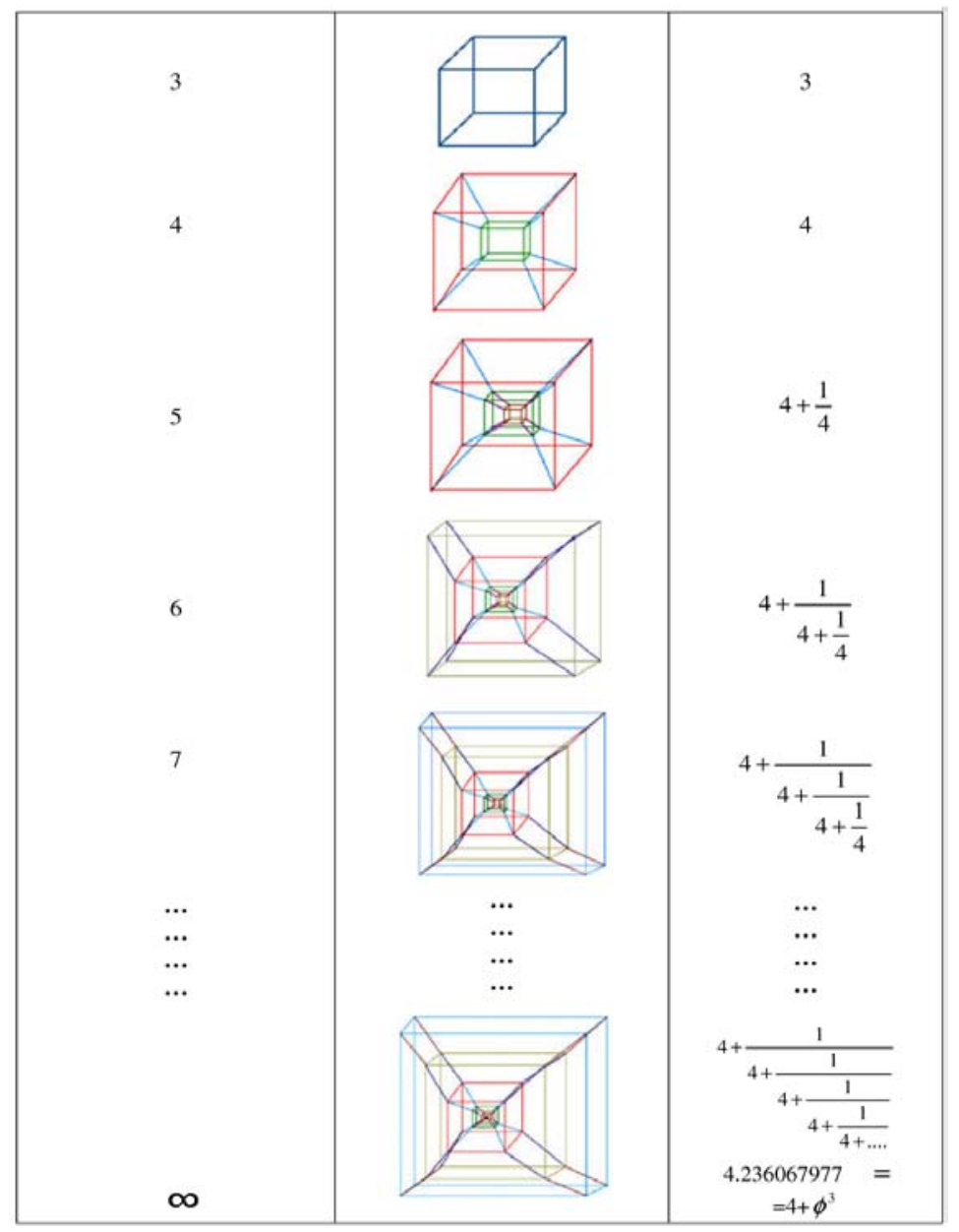

Figure 2 : A Euclidean representation of [einfinity-spacetime] $\mathcal{E}^{\infty}$ fractal spacetime as an infinite sequence of nested four dimensional hypercubes (redrawn from [16])

The 4-dimensional hypercube is the Euclidean representation of the E-infinity universe. It is a challenge to represent $\mathcal{E}^{\infty}$ in its proper non-Euclidean form.

Another mathematical property of the Cantor set is that its cardinality (number of points or elements) is exactly the same as the original continuous line. Thus, the Cantor set is a perfect compromise between the discrete and the continuum; it is a discrete structure that has the same number of elements as the continuum.
Mathematically, the $\mathcal{E}^{\infty}$ universe is a random Cantor set extended to infinite dimensions. Remarkably, the Hausdorff dimension of this infinite extension is no larger than $4+\phi^{3}$.

Figure 3 illustrates the steps involved in deriving $\mathcal{E}^{\infty}$ universe. Recall that the standard Cantor set is obtained by dividing the unit interval into three parts and removing the middle part, leaving the end points. Then do the same to each of the two remaining parts. We 
repeat the process infinitely often, and in the end there remain only isolated points, or 'Cantor dust'. The Menger-Urysohn dimension is 0 , but the Hausdorff dimension is $\log 2 / \log 3$.

If however at each step we remove not necessarily the middle section but any one of the three chosen at random, then again we are left with isolated points but now the Hausdorff dimension is the golden mean $\phi=(\sqrt{5}-1) / 2=0.61803398 \ldots$. This Important result, proven by Mauldin and Williams in 1986 [18], is what makes it possible to derive the $\mathcal{E}^{\infty}$ universe with all its remarkable properties, as we shall see.

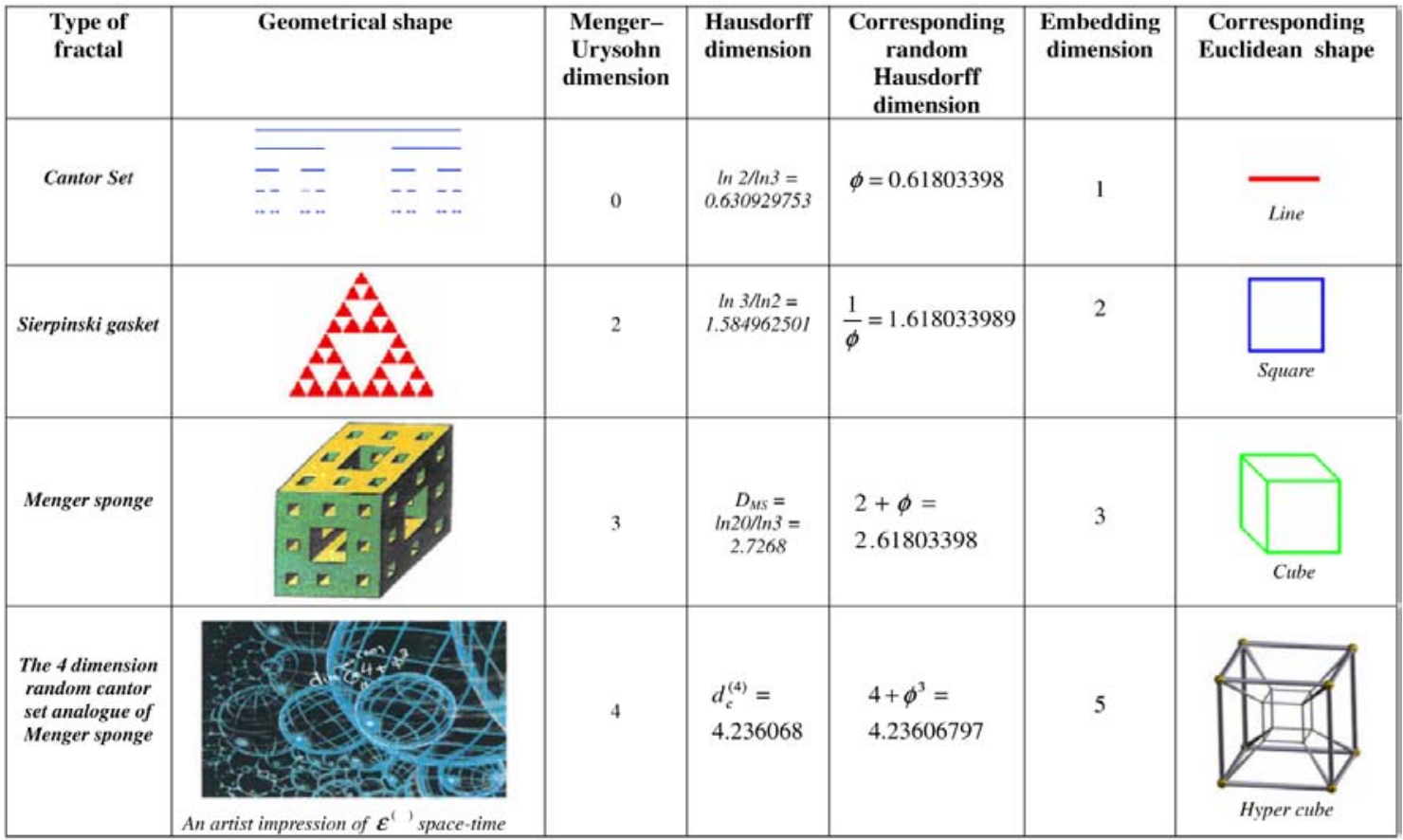

Figure 3 : Fractals and dimensions in the derivation of $E$ infinity spacetime

We now construct the higher dimensional random Cantor spaces [16] (see Fig. 3). The 2dimensional version is the Sierpinski gasket, with Hausdorff dimension of $1 / \phi \approx 1.61803398$; the 3 dimensional version is the Menger sponge, with Hausdorff dimension $2+\phi \approx 2.61803398$. These are both well-known geometric shapes. The 4-dimensional version, with Hausdorff dimension $4+\phi^{3} \approx 4.2360679$, is given only an artist's representation. The 4-dimensional version is the same as the $\mathcal{E}^{\infty}$ universe constructed from an infinite number of random Cantor sets, as will be made clear later. Note that the diagram representing the 4-dimensional Cantorial spacetime is space-filling with smaller and smaller spheres. This space-filling property makes the Cantorian spacetime non-differentiable and discontinuous, yet dense everywhere in spacetime. It recalls the quasi-periodic Penrose tiling in 2 dimensions of Euclidean (flat) space where the golden mean is key (see [19] and references therein). Branching processes based on the golden mean are also space-filling [20], as are spiral leave arrangement patterns with the golden angle between successive leaf primordial (see [21]).

$\mathrm{MeN}$ has presented several different formal derivations of $\mathcal{E}^{\infty}$ spacetime; we give here the simplest [16],[17] which is based on the mathematical properties of Borel sets to which Cantor sets belong.

The expectation value of the Hausdorff dimension of the Cantor set extended to infinity is simply a sum over $n$, for $n=0$ to $n=\infty$, of $n$ multiplied by the Hausdorff dimension of the random Cantor set raised to the power $n$.

$$
<\operatorname{Dim} E-\infty>=\sum_{0}^{\infty} n\left(d_{c}^{(0)}\right)^{n}
$$

where the superscript in $d_{c}^{(0)}$ refers to the MengerUrysohn dimension of the random Cantor set, which is 0 , while the corresponding Hausdorff dimension $d_{c}^{(0)}$ is $\phi$.. Summing up the infinite number of terms gives the answer $4+\phi^{3}$ exactly, as follows:

$$
\begin{aligned}
<\operatorname{Dim} E-\infty> & =(0)+(1)(\phi)^{1}+(2)(\phi)^{2}+(3)(\phi)^{3}+\ldots \\
& =4+\phi^{3} \\
& =4.236067977 \ldots
\end{aligned}
$$


The intersection rule of sets, the 'bijection formula', relates the Menger-Urysohn dimension to the Hausdorff dimension. It shows that we can lift $d_{c}^{(0)}$ to

$$
d_{c}^{(n)}=\left(1 / d_{c}^{(0)}\right)^{n-1}
$$

Taking $d_{c}^{(0)}=\phi$, and lifting to $n=4$ dimensions gives

$$
d_{c}^{(4)}=\left(1 / d_{c}^{(0)}\right)^{4-1}=4+\phi^{3}=1 / \phi^{3}=4.236067977=<\operatorname{Dim} E-\infty>_{H}
$$

Thus the expectation value of the Hausdorff dimension of $\mathcal{E}^{\infty}$ universe is the same as that of a universe with a Menger-Urysohn dimension of 4 . That is why $\mathcal{E}^{\infty}$ is a hierarchical universe that looks and feels 4 dimensional.

\section{Vi. How $\mathcal{E}^{\infty}$ Relates to Penrose Tiling, And The Fi-Bonacci Sequence}

$\mathcal{E}^{\infty}$ universe connects with Penrose tiling and the Fibonacci sequence (see [19]) through $\mathcal{E}^{\infty}$ [17], [21] and the golden mean. The golden mean is an irrational number and like any irrational number it can be approximated by a fraction; for example $\frac{22}{7}$ is quite close to $\pi$. The usual way of obtaining such an approximation, using continued fractions, converges more slowly for $\phi$ than for any other number, and in this sense $\phi$ is the most irrational number there is.

In Noncommutative Geometry [23], Alain Connes identified Penrose's fractal tiling as a mathematical quotient space (a space of points 'glued together' by an equivalence relationship), with the dimensional function:

$$
D(a, b)=a+b \phi
$$

where $a, b$ are integers and $\phi=(\sqrt{5}-1) / 2$. Writing $D_{n}\left(a_{n}, b_{n}\right)$ where both $a_{n}$ and $b_{n}$ satisfy the Fibonacci recurrence relation $x_{n+2}=x_{n}+x_{n+1}$ and starting with $D_{0}=D(0,1) \quad$ and $D_{1}=D(1,0)$, the following dimensional hierarchy is obtained:

$$
\begin{aligned}
D_{0} & =D(0,1)=0+\phi=\phi \\
D_{1} & =D(1,0)=1+(0) \phi=1 \\
D_{2} & =D(0+1,1+0)=1+\phi=1 / \phi \\
D_{3} & =D(1+1,0+1)=2+\phi=(1 / \phi)^{2} \\
D_{4} & =D(1+2,1+1)=3+2 \phi=(1 / \phi)^{3} \\
D_{5} & =D(2+3,1+2)=5+3 \phi=(1 / \phi)^{4} \\
D_{n}\left(a_{n}, b_{n}\right) & =D\left(\left(a_{n-1}, a-n-2+\left(b_{n-1}+b_{n-2}\right)\right) \phi=(1 / \phi)^{n-1}\right.
\end{aligned}
$$

It is notable that for $\mathrm{D}_{4}$ (dimension 4), the Fibonacci number is $\left.(1 / \phi)^{3}=4+\phi^{3}\right)$, exactly the Hausdorff dimension of a Menger-Urysohn 4dimensional space.

By induction,

$$
D_{n}=(1 / \phi)^{n-1}
$$

and we get back the bijection formula from $\mathcal{E}^{\infty}$ algebra (see Eq (3) above):

$$
d_{c}^{(n)}=(1 / \phi)^{n-1}
$$

Summing random Cantor sets to infinity in the creation of $\mathcal{E}^{\infty}$ universe is evocative of spacetime being created by actions over all scales, from submicroscopic to macroscopic and beyond, as envisaged The Rainbow and the Worm, the Physics of Organisms by one of us (MWH) [24], following Whitehead [2] and Wolfram Schommers [25].
MeN has conjectured that $\mathcal{E}^{\infty}$ spacetime can also resolve major paradoxes within quantum theory and produce new results as described elsewhere ([26],[27],[28]).

Here, we move on to the role of cycles in the organization of spacetime and why the golden mean seems to be built into the fabric of life and the universe.

\section{Vil. Cycles Everywhere for Stability and AUTONOMY}

Nature abounds with cycles and oscillations, from subatomic vibrations to planetary motion, solar cycles and galactic rotations. Some, like Penrose and Gurzadyan hold that the Universe cycles through deaths and rebirths, based on data collected by NASAs WMAP (Wilkinson Microwave Anisotropy Probe) and the BOOMERanG balloon experiment in Antarctica [29]. In a recent review, $\mathrm{MWH}$ [31]considers the importance of cycles and the golden mean for natural processes. We 
provide a brief account here and in the following two sections.

Cycles are intimately tied to the study of dynamical systems, beginning with celestial mechanics. Newton tried to describe the planetary cycles in terms of his laws of motion more than 300 years ago.

Dynamical systems can be treated mathematically as oscillators. A harmonic oscillator has a certain natural frequency. When perturbed by an external force with the same frequency, resonance occurs and the motion of the oscillator becomes unbounded or unstable. For a typical nonlinear oscillator, this happens whenever the frequency of the perturbing force is a rational multiple of the natural frequency of the oscillator.

Andrey Kolmogorov (1903-1987), Vladimir Arnold (1937-2010) and Jürgen Moser (1928-1999) were responsible for the the Kolmogorov Arnold and Moser (KAM) theorem. The KAM theorem is very important for understanding how cyclic activities (or oscillators) interact with one another [30].

They were investigating the behaviour of integrable Hamiltonian systems. The trajectories of Hamiltonian systems in phase space are confined to a doughnut shaped surface, an invariant torus. Different initial conditions will trace different invariant concentric tori in phase space, separated by unstable chaotic regions, where the motion is irregular and unpredictable.

An important consequence of the KAM theorem is that for a large set of initial conditions, the motion remains perpetually quasi-periodic, and hence stable. KAM theory has been extended to non-Hamiltonian systems and to systems with fast and slow frequencies.

The KAM theorem become increasingly difficult to satisfy for complex systems with more degrees of freedom; as the number of dimensions of the system increases, the volume occupied by the tori decreases. Those KAM tori that are not destroyed by perturbation become invariant Cantor sets, or Cantori; and the frequencies of the invariant Cantori approximate the golden mean [32].

The golden mean effectively enables multiple oscillators within a complex system to co-exist without blowing up the system. But it also leaves the oscillators within the system free to interact globally (by resonance), which may have important applications even in the study of the brain (see later). To get a better picture, we look at the circle map.

\section{Vili. Cycles, Quasi-Periodicity, Golden Mean and Chaos}

Cycles are often represented by the circle map, a graph that maps the circle onto itself. The simplest form is [33],[34]:

$$
\theta_{n+1}=\theta_{n}+\Omega(K / 2 \pi) \sin 2 \pi \theta_{n}
$$

where the variable $\theta_{n+1}$ is computed mod 1 (meaning counting only partial circles, as full circles get back to the starting point), $K$ is the coupling strength and $\Omega$ is the external driving frequency. This map is used to describe oscillatory systems from solid state physics to heart rhythms. The circle map tracks the universal behaviour of dynamical systems associated with transition from cycles to chaos via quasi-periodicity.

The most studied circle map involves a ratio of basic frequencies $w=\phi=(\sqrt{5}-1) / 2$, the golden mean critical point at $K=1$ and $\Omega=\Omega_{c}=0.60666106347011$ $(\approx \phi=0.618033989 \ldots)$, reported in many experiments.

Circle maps contain some key features. Arnold tongues are regions in the phase space of circle maps with locally constant rational rotation (winding) numbers between the driver and the natural oscillator frequencies, $p / q$. They were first investigated for a family of dynamical systems defined by Kolmogorov, who proposed this family as a simplified model for driven mechanical rotors described by equation (9). The circle map of the equation exhibits certain regions in the parameters space when it is locked to the driving frequency (phase- locked, or synchronized). Here, $\theta$ is interpreted as the polar angle such that its value lies between 0 and 1 ; the two parameters are $K$, the coupling strength between the driver and the rotor, and $\Omega$, the driver frequency. A typical map with Arnold tongues is given in Figure 4 . 


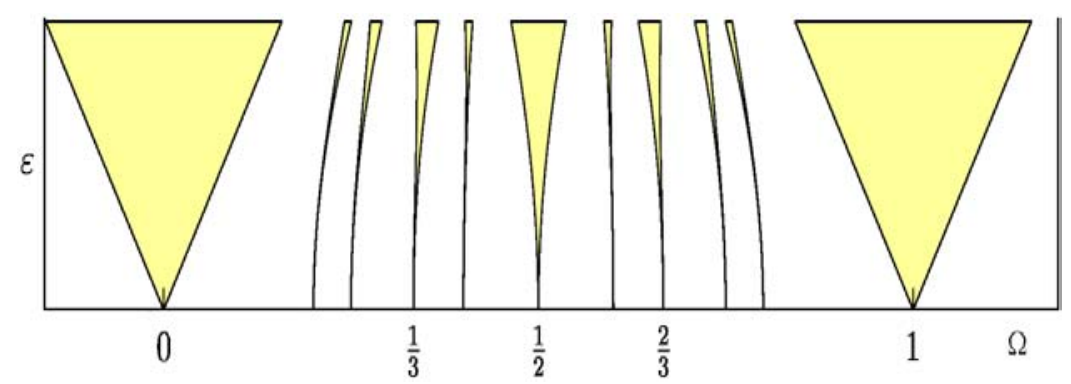

Figure 4 : Some Arnold tongues in the standard circle map $\epsilon=K / 2 \pi$ versus $\Omega$

For small to intermediate values of $K,(0<K<$ 1 ), and certain values of $\Omega$, the map exhibits phase locking. In the phase-locked regions, $\theta_{n}$ advances essentially in rational multiples of $n$; although it may do so chaotically on the small scale.

The phase-locked regions, called Arnold tongues, are shaded yellow in Figure 4; while the quasiperiodic regions are white. Each yellow $\mathrm{V}$ region touches down to a rational value of $\Omega=p / q$ in the limit as $K \rightarrow 0$. The values of $(K, \Omega)$ in one of these regions will all result in a motion with rotation number $w=\frac{p}{q}$. For example, all values of $(K, \Omega)$ in the middle $\mathrm{V}$ region correspond to $w=\frac{1}{2}$. In other words, the sequence stays locked on to the signal despite significant noise or perturbation. This ability to lock on in the presence of noise is central to the utility of phase-locked loop electronic circuits.
The circle map in Figure 4 is invertible or symmetrical around the mid line. For $K>1$, the circle map is no longer invertible. In Figure 5a [35], the circle map is extended to $K=4$. Arnold tongues of synchronization are in grey with winding numbers indicated inside the tongue. The white regions are quasiperiodic, and the stippled regions appearing beyond the line $K=1$ represent chaos. This map also depicts fractal self-similarity on different scales. Fractal self-similarity and chaos are closely related. A chaotic system has a fractal dimension and exhibits selfsimilarity over many scales.

Chaos does not mean random. Mathematically, chaos is sensitive to initial conditions), but it is globally determined in the sense that tends toward a strange attractor (see Appendix 2)
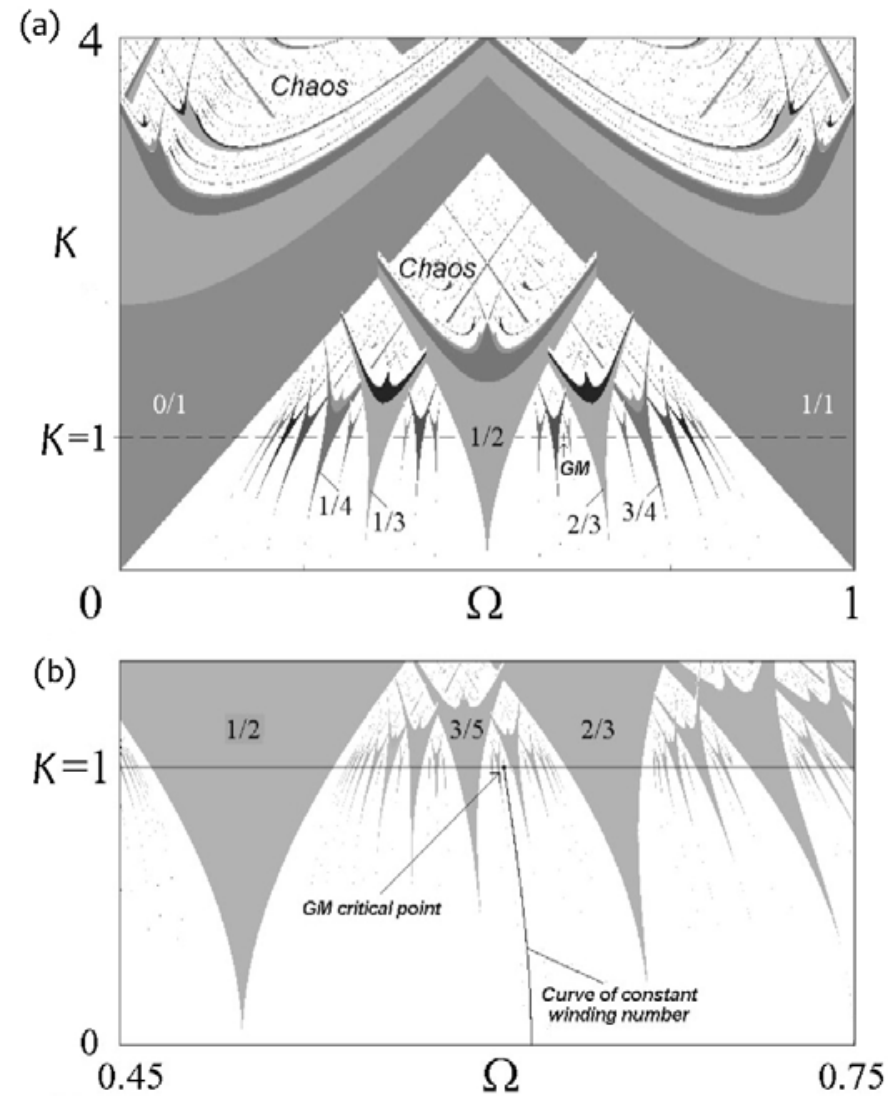

Figure 5 : Extended circle map (see text for details) 
The golden mean critical point (GM) is where the curve of constant irrational winding number $\phi=(\sqrt{5}$ -1 ) $/ 2$ terminates on the line $K=1$ (see Fig $5 b$ ), and quasi-periodic behaviour undergoes transition to chaos. This point is marked by an infinite sequence of unstable orbits with periods given by the Fibonacci numbers.

The golden mean is thus located at the edge of chaos, and has a role in keeping the system of oscillators active without interfering with one another as well as away from the state of chaos.

There are claims that the planetary orbits around the sun exhibit golden ratios or ratios according to the Fibonacci sequence numbers, as many people have commented (see [36] [37] for example). So is our solar system stable? The question is whether it will remain stable as such, at least for billions of years, or transit to chaos much sooner than that. Some astrophysicists claim however, that the planetary orbits are chaotic the and sensitive to initial conditions but this means only that they are unpredictable for longer than 100 million years into the future [38] so there is no immediate cause for alarm.

\section{iX. Chaos and Strange Attractors}

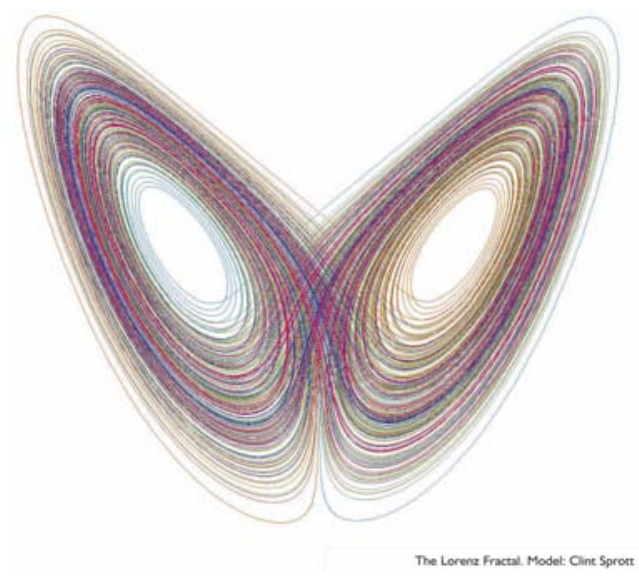

Figure 6: The Lorenz attractor (from [40])

Edward Lorenz is the generally acknowledged father of chaos theory [39]. According to one account, during the winter of 1961, Lorenz was running a climate model on the computer described by 12 differential equations, when he decided to repeat one of the runs with a small change. Instead of calculating to six decimal digits, he rounded that to three to save computing time, fully expecting to get the same results. But he didn't. That was the beginning of his discovery of the sensitive dependence on initial conditions of chaotic systems, which he described as the butterfly effect. It makes long term weather prediction impossible. His toy equations produced the Lorenz attractor (Figure 6) (the prototype of strange attractors associated with chaotic systems), which bears serendipitous resemblance to butterfly wings, and became the emblem of the chaos era that followed. Numerous strange attractors have been created, mostly as computer artwork, beginning with the book by Clint Sprott [40]. But chaos theory has found applications in meteorology, physics, engineering, economics, biology and medicine.

The Lorenz attractor is a fractal, with self-similar structure on different scales. It has a fractal dimension of 2.06215 and lives in a space of at least 3 dimensions. It contains unstable periodic orbits that can be identified using various mathematical procedures [41], and can also be regarded as twisted or knotted periodic orbits [42].
Chaos theory has been taken up enthusiastically in every field including quantum physics, in the form of quantum chaos, which tries to build a bridge between chaos in classical mechanics and the wavelike motion of electrons in atoms and molecules. Martin Gutzwiller wrote [43]: "The phase space for a chaotic system can be organized at least partially around periodic orbits, even though they are sometimes quite difficult to find."

Chaos is typically found in turbulent flows of fluids, gases, and the atmosphere. Turbulence is traditionally regarded as one of the most intractable problems in physics and mathematics. Mary Selvam first proposed a theory of turbulent fluid flow based on fractal spacetime fluctuations in 1990 (see [44]).

Selvam treats the fractal fluctuations on all spacetime scales as a superposition of a continuum of eddies or vortices. Large scale fluctuations result from the integration of smaller scale fluctuations within, and the growth trajectory traces an overall logarithmic spiral ow path with the quasi-periodic Penrose tiling pattern for internal structure.

The ratio of radii or circulation speeds corresponding to the successive growth steps of the large eddy generating the geometry of the quasiperiodic Penrose tiling pattern is, of course, equal to the golden mean $\Phi=1.618 \ldots$..[31]). 
Treating turbulence as a continuum of discrete eddies or cycles with Penrose tiling pattern of growth captures key features of biological spacetime, as we shall see.

\section{Quantum Coherence and Circular Thermody-NAMICS OF ORganisms}

In The Rainbow and the Worm, the Physics of Organisms [24], MWH presented empirical evidence and theoretical arguments suggesting that organisms are quantum coherent, and derived a circular thermodynamics of organisms that enables organisms to transform and transfer energy with minimum dissipation, which is also implied by quantum coherence. Here, we concentrate on the circular thermodynamic, which depends on a coherent fractal organization of biological spacetime.

The first thing to note is that organisms do not make their living by heat transfer. They are not heat engines, but isothermal systems far away from thermodynamic equilibrium, and depend on the direct transfer of molecular energy by proteins and other macromolecules acting as quantum molecular energy machines at close to $100 \%$ efficiency [24].

(It is well-known that enzymes speed up chemical reactions in organisms by a factor of $10^{10}$ to $10^{23}$ [45], but they cannot do that without the water that constitutes 70 to $80 \%$ of cells and tissues. It is widely recognized that water gives exibility to proteins, reduces the energy barrier between reactants and products and increases the probability of quantum tunnelling by a transient compression of the energy barriers. But there is more to how water actually organizes enzyme reactions in living organisms. Findings within the past decade suggests that interfacial water associated with macromolecules and membranes in cells and tissues is in a quantum coherent liquid crystalline state, and plays a lead role in creating and maintaining the quantum coherence of organisms, as elaborated in Living Rainbow $\mathrm{H}_{2} \mathrm{O}$ [46] and elsewhere [47],[48].)

For isothermal processes, the change in Gibbs free energy $\Delta G$ (thermodynamic potential for doing work at constant temperature and pressure) is,

$$
\Delta G=\Delta H-T \Delta S
$$

where $\Delta H$ is the change in enthalpy (heat content), $T$ is temperature in deg $\mathrm{K}$, and $\Delta S$ is the change in entropy.

Thermodynamic efficiency requires that $\Delta S$ approaches 0 (least dissipation) and $\Delta H=0$, or $\Delta G=0$ via entropy-enthalpy compensation, i.e., entropy and enthalpy changes cancelling each other out. We shall see how the organism accomplishes that.

\section{Xi. A Fractal Hierarchy of Coupled CyCles}

For a system to keep far away from thermodynamic equilibrium - death by another name - it must capture energy and material from the environment to develop, grow and recreate itself from moment to moment in a life-cycle, to reproduce and provide for future generations (Figure 7). The key to understanding the thermodynamics of the living system is not so much energy flow as energy capture and storage to create a reproducing life-cycle. The dynamic closure implied by the life-cycle is the beginning of a circular thermodynamics that transforms and transfers energy and materials with maximum efficiency (least dissipation) (see [24], [49]).
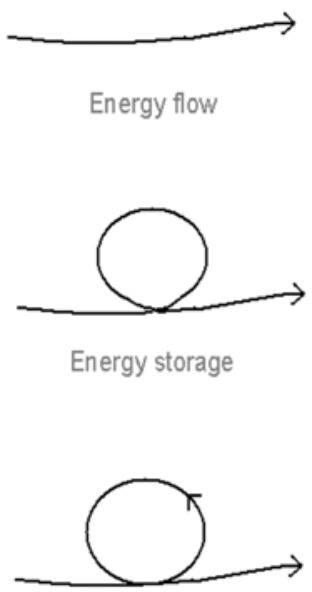

Reproducing life cycle coupled to energy flow

Figure 7 : Life depends on energy capture and storage to form a self-reproducing life-cycle coupled to energy flow

The life-cycle is a fractal hierarchy of self-similar cycles organised by the characteristic spacetimes of the processes involved. All real processes have characteristic spacetimes. In the organism, the heart $\left(10^{-1} \mathrm{~m}\right)$ beats in a second, nerve cells $\left(10^{-4} \mathrm{~m}\right)$ fire in a tenth of a second or faster, and protons $\left(10^{-15} \mathrm{~m}\right)$ and electrons $\left(10^{-17} \mathrm{~m}\right)$ move in $10^{-12}$ to $10^{-15} \mathrm{~s}$. Cells divide in minutes, and physiological processes have longer cycles of hours, a day, a month or a year.

The coherent fractal hierarchy of living activities arises because processes with matching space times interact most strongly through resonance, and also link up to the entire hierarchy. That is why biological activities come predominantly in cycles or biological rhythms. (In the language of quantum physics, the organism is a superposition of coherent quantum activities over all spacetimes [24].) The possibility for cycles in the living world coupling and linking up to cycles in the physical universe is surely why life is 
possible, and indeed some would argue, as Whitehead did [2], that the entire universe is alive.

The coupled cycles form a nested fractal selfsimilar structure. The lifecycle consists of smaller cycles each with still smaller cycles within, spanning characteristic spacetimes from sub-nanometre to metres and from $10^{-15}$ s to hours and years (Figure 8).

Figure 8 : The fractal structure of coupled activity cycles in living systems

\section{Xil. Minimum Entropy Production}

Cycles enable the activities to be coupled together, so that energy yielding processes can transfer energy directly to those requiring energy; and the direction can be reversed when necessary. This cooperativity and reciprocity resulting from the fractal hierarchy of coupled cycles is an extended form of Onsager reciprocity relation that conventionally applies strictly only to near-equilibrium steady state (see [24] for details). What it means in practice is that energy can be concentrated to any local point where it is needed, and conversely spread globally from any local point. In that way, the fractal hierarchy of coupled cycles maximizes both local autonomy and global cohesion, which is the hallmark of quantum coherence [24] according to the criterion of factorisability defined by Glauber [50].

To get an idea of such coupled cycles, one needs to look no further than charts of biochemical metabolic pathways [51]. Most if not all of the reactions go either way, depending on the local concentrations of reactants and products. In further accord with circular thermodynamics, biochemical recycling is ubiquitous; there are numerous scavenging or salvaging pathways for the recovery of building blocks of proteins, nucleic acids, glycolipids, and even entire proteins.

The fractal hierarchy of coupled cycles confers dynamic stability as well as autonomy to the system on every scale. Thermodynamically, no net entropy is generated in the case of perfect cycles; and the system maintains its organization.
The fractal structure effectively partitions the organism into a hierarchy of systems within systems defined by the extent of equilibration of (dissipated) energies. Thus, energies equilibrated or evenly spread within a smaller spacetime will still be out of equilibrium in the larger system encompassing the first, and hence capable of doing work.

There are now two ways to mobilize energy efficiently with entropy change approaching zero: very slowly with respect to the characteristic time so it is reversible at every point, or very rapidly with respect to the characteristic time, so that in both cases the energy remains stored (in a coherent non-degraded form) as it is mobilized. Consequently, the organism simultaneously achieves the most efficient equilibrium and far-from-equilibrium energy transfer.

The nested dynamical structure also optimises the kinetics of energy mobilisation. For example, biochemical reactions depend strictly on local concentrations of reactants, which are extremely high, as their extent of equilibration is typically at nanometre dimensions (in nanospaces).

In the ideal - approached most closely by the healthy mature organism and the healthy mature ecosystem - an overall internal conservation of energy and compensation of entropy $(\Sigma \Delta S=0)$ is achieved. In this state of balance, the system organization is maintained and dissipation minimized; i.e., the entropy exported to the environment also approaches zero, $\Sigma \Delta S$ $\geq 0$ (Figure 9).

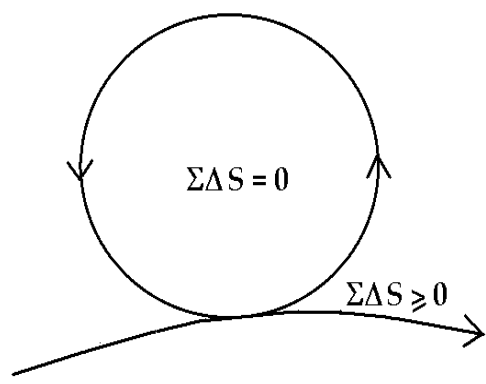

\section{Figure 9 : The zero-entropy ideal of circular thermodynamics}

Internal entropy compensation (and energy conservation) implies that there needs to be free variation in microscopic states within the macroscopic system; i.e., the internal microscopic detailed balance at every point of classical steady state theory is violated. (This is also the basis of the extension of Onsagers reciprocity relationship to far from equilibrium condition.)

For an organism, this means that detailed energy balance is not necessary at every point. Most often, parts of it are in deficit, and severely so, when one needs to run from a tiger knowing the energy can be replenished after a successful escape. The same applies to ecosystems: all species are in a sense storing 
energy and resources (nutrients) for every other species via complex food webs and other symbiotic relationships.

The above considerations give rise to the prediction that a sustainable system maximizes cyclic, non-dissipative flows while minimizing dissipative flows, i.e., it tends towards minimum entropy production even under far from equilibrium conditions, as conjectured by llya Prigogine [52].

Such a system has a hierarchy of coherent energy storage spacetimes so that within the coherence volume, there is no time separation, and within the coherence time, there is no space separation. Thus, organic spacetime exists as a hierarchy of simultaneities, or durations, precisely as envisaged by Bergson [5] and Whitehead [2],[6]. (See [24] for a more detailed discussion).

The golden mean most likely enters into the fractal structure in the form of the golden fractal. As the most irrational of all numbers (see Section 6 above), it allows the maximum number of non-resonating activities to co-exist (representing maximum coherent energy storage). On the other hand, it is also arbitrarily close to a maximum number of rational numbers so specific resonances can easily be established for energy transfer.

We shall see more clearly how the golden fractal of neuronal activities are the key to optimum intercommunication and information processing in the final section. The next section shows how fractals are mathematically isomorphic to quantum coherent states.

\section{Xiil. Fractals and Quantum Coherence}

The coherent fractal structure maximizes global connectivity and local autonomy, the hallmark of quantum coherence, as mentioned earlier. One of the authors (GV) has shown that a functional representation of self-similarity - the most important property of fractal structures - is mathematically isomorphic with squeezed quantum coherent states $[53,54,55]$, in which Heisenberg's uncertainty is minimum. Quantum coherence thus seems to underly the ubiquitous recurrence of fractal self-similarity in Nature.

Let us see how this works with the logarithmic spiral, and the special case of the golden spiral, as an example. The golden spiral and its relation to the Fibonacci sequence are of great interest because the Fibonacci sequence appears in many phenomena, ranging from botany to physiological and functional properties in living systems, as the "natural" expression in which they manifest themselves.

The defining equation for the logarithmic spiral in polar coordinates $(r, \theta)$ is $[56,57]$ :

$$
r_{0} e^{d \theta}
$$

with $r_{0}$ and $d$ arbitrary real constants and $r_{0}>0$. In a log-log plot with abscissa $\theta=\ln e^{\theta}$ the spiral is represented by a straight line with slope $d$

$$
\ln \frac{r}{r_{0}}=d \theta \text {. }
$$

The (fractal) self-similarity property of the logarithmic spiral is manifest in the constancy of the angular coefficient tan ${ }^{-1} d$ : rescaling $\theta \rightarrow n \theta$ affects $r / r_{0}$ by the power $\left(r / r_{0}\right)^{n}$.

The logarithmic spiral is called the golden spiral when at $\theta=\pi / 2$ we have $r / r_{0}=e^{d(\pi / 2)}=\Phi$, where $\Phi$ denotes the golden mean $(1+\sqrt{5}) / 2$. We may introduce the subscript $g$ (denoting golden) and put $d_{g} \equiv(\log \Phi) /(\pi / 2)$. The polar equation for the golden spiral is thus $r_{g}(\theta)=r_{0} e^{d_{g} \theta}$. The radius of the golden spiral increases in geometrical progression with the ratio $\Phi$ as $\theta$ increases by $\pi / 2: r_{g}(\theta+n \pi / 2)=r_{0} e^{d_{g}(\theta+n \pi / 2)}$ $=r_{0} e^{d_{g} \theta} \Phi^{n}$ and $r_{g, n} \equiv r_{g}(\theta=n \pi / 2)=r_{0} \Phi^{n}, n=0$, $1,2,3, \ldots$.

The so called Fibonacci tiling provides a tool for a good approximate construction of the golden spiral. The Fibonacci tiling is obtained by drawing in an appropriate way [56] squares whose sides are in the Fibonacci progression, $1,1,2,3,5,8,13, \ldots$ (the sequence is defined by the relation $F_{n}=F_{n-1}+F_{n-2}$, with $\left.F_{0}=0 ; \quad F_{1}=1\right)$. The Fibonacci spiral is then made from quarter-circles tangent to the interior of each square. It does not coincide exactly with the golden spiral because $F_{n} / F_{n-1} \rightarrow \Phi$ in the $n \rightarrow \infty$ limit, but is not equal to $\Phi$ for $n$ finite.

The golden ratio $\Phi$ and its "conjugate" $\psi \equiv 1-$ $\Phi=-1 / \Phi=(1-\sqrt{5}) / 2=-\phi$ are both solutions of the quadratic equation

$$
x^{2}-x-1=0
$$

and of the recurrence equation $x^{n}-x^{n-1}-x^{n-2}=0$, which, for $n=2$, is the relation (13). This is satisfied also by the geometric progression with ratio $\Phi$ of the radii $r_{g, n}=r_{0} \Phi^{n}$ of the golden spiral. Eq. (13) is the characteristic equation of the differential equation $r+\dot{r}-r=0$, which admits as solution $r(t)=r_{0} e^{i \omega t}$ $e^{+d \theta(t)}$ with $\omega= \pm i \sqrt{5} / 2$ and $\theta=-t /(2 d)+c$ with $c, r_{0}$ and $d$ constants. By setting $c=0, r(t)=r_{0} e^{\mp \sqrt{5} t / 2}$ $e^{-t / 2}$, i.e. $r_{\Phi}(t)=r_{0} e^{-\Phi t}$ and $r_{\psi}(t)=r_{0} e^{-\psi t}$.

Consider now the parametric equations of the logarithmic spiral Eq. (11):

$$
\begin{aligned}
& x=r(\theta) \cos \theta=r_{0} e^{d \theta} \cos \theta \\
& y=r(\theta) \sin \theta=r_{0} e^{d \theta} \sin \theta
\end{aligned}
$$

The point $z=x+i y=r_{0} e^{d \theta} e^{i \theta}$ on the spiral is completely specified in the complex $z$-plane only when the sign of $d \theta$ is assigned. The completeness of the (hyperbolic) basis $\left\{e^{-d \theta}, e^{+d \theta}\right\}$ requires that both

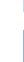


the factors $q=e^{ \pm d \theta}$ must be considered. In many instances the so-called direct $(q>1)$ and indirect $(q<1)$ spirals are both realized in the same system (e.g. in phyllotaxis studies).

We thus consider the points $z_{1}=r_{0} e^{-d \theta} e^{-i \theta}$ and $z_{2}=r_{0} e^{+d \theta} e^{+i \theta}$. For convenience (see below) opposite signs for the imaginary exponent $i \theta$ have been chosen. Using the parametrization $\theta=\theta(t), z_{1}$ and $z_{2}$ are found to be solutions of the equations

$$
\begin{aligned}
& m \ddot{z}_{1}+\gamma \dot{z}_{1}+\kappa z_{1}=0, \\
& m \ddot{z}_{2}-\gamma \dot{z}_{2}+\kappa z_{2}=0
\end{aligned}
$$

respectively, provided the relation

$$
\theta(t)=\frac{\gamma}{2 m d} t \equiv \frac{\Gamma}{d} t
$$

holds (where we have neglected an arbitrary additive constant). We see that $\theta(T)=2 \pi$ at $T=2 \pi d / \Gamma$. In Eqs. (15) a dot denotes derivation with respect to $t ; m, \gamma$ and $\kappa$ are positive real constants. The notations $\Gamma \equiv \gamma / 2 m$ and $\Omega^{2}=(1 / m)\left(\kappa-\gamma^{2} / 4 m\right)=\Gamma^{2} / d^{2}$, with $\kappa>\gamma^{2} / 4 m$, have been used. The parametric expressions $z_{1}(t)$ and $z_{2}(t)$ for the logarithmic spiral are thus $z_{1}(t)=r_{0} e^{-i \Omega t} e^{-\Gamma t}$ and $z_{2}(t)=r_{0} e^{+i \Omega t}$ $e^{+\Gamma t}$, solutions of Eqs. (15a) and (15b). At $t=n T, z_{1}=$ $r_{0}\left(e^{-2 \pi d}\right)^{n}, z_{2}=r_{0}\left(e^{2 \pi d}\right)^{n}$, with the integer $n=1$, $2,3 \ldots$

This suggests that $t$ can be interpreted as the time parameter and the time-evolution of the direct and indirect spirals is described by the equations (15a) and (15b). The "two copies" $\left(z_{1}, z_{2}\right)$ can be viewed as the evolution forward in time and backward in time, respectively. The "angular velocity" of (the growth of) the spiral is given by $|d \theta / d t|=|\Gamma / d|$.

By putting $\left[z_{1}(t)+z_{2}^{*}(-t)\right] / 2=x(t) \quad$ and $\left[z_{1}^{*}(-t)+z_{2}(t)\right] / 2=y(t)$, we reduce eqs. (15a) and (15b) to the pair of damped and amplified harmonic oscillators:

$$
\begin{aligned}
& m \ddot{x}+\gamma \dot{x}+k x=0, \\
& m \ddot{y}-\gamma \dot{y}+k y=0
\end{aligned}
$$

respectively. The second of these is the time-reversed image ( $\gamma \rightarrow-\gamma$ ) of the first and the global system $(x-y)$ is a closed system. We see that the oscillator $x$ is a dissipative, open (non-hamiltonian) system and in order to set up the canonical formalism we need to double the degrees of freedom by introducing its time reversed image $y$ [58]: the physical meaning of the requirement to consider both the elements of the basis $\left\{e^{-d \theta}, e^{+d \theta}\right\}$ is that we need to consider the closed system $\left(z_{1}, z_{2}\right)$. Only then can we set up the canonical formalism.

If we did not close the system $z_{1}$ with the system $z_{2}$, we would not be able to define the conjugate momenta because the Lagrangian would not exist.
We now remark that classical, deterministic systems presenting loss of information (dissipation) might, providing some constraints are imposed, behave according to quantum evolution [59, 60, 61]. In the present case, this means that the logarithmic spiral and its time-reversed double (the direct and the indirect spiral) manifest themselves as macroscopic quantum systems. The quantization of the system described by Eqs. (15a) and (15b) (or (17a) and (17b)), can be performed in the usual way and the result is that the system ground state $|0(t)\rangle$ is a coherent squeezed state, which is produced by condensation of couples of (entangled) $A$ and $B$ modes (related to the $\left(z_{1}, z_{2}\right)$ couple, see Ref. [58, 54, 55]) for details): $(A B)^{n}, n=0$, $1,2 \ldots \infty$. The operator which generates $|0(t)\rangle$ is recognized to be the two mode squeezing generator with squeezing parameter $\zeta=-\Gamma t$. It must be stressed that the correct mathematical framework to study quantum dissipation is the one of quantum field theory (QFT). The realization of the logarithmic spiral (and in general of fractals) in the many cases observed in nature involves indeed an infinite number of elementary degrees of freedom.

We may define the fractal Hamiltonian, which turns out to be actually the fractal free energy for the coherent boson condensation process out of which the fractal is formed. We indeed find that time evolution is controlled by the entropy operator. This is to be expected because entropy controls time irreversibility and the breakdown of time-reversal symmetry that is characteristic of dissipation is clearly manifest in the formation process of fractals. In the case of the logarithmic spiral, the breakdown of time-reversal symmetry is associated with the chirality of the spiral: the indirect (right-handed) spiral is the time-reversed, but distinct, image of the direct (left-handed) spiral.

These results can be also extended to other examples of deterministic fractals [54], such as the Koch curve, the Sierpinski gasket and carpet, the Cantor set, etc..We do not consider these cases here. We only recall that by resorting to the results reported above the isomorphism can be shown [55] to exist between classical and quantum electrodynamics, from one side, and the fractal self-similarity structure and squeezed coherent states, from the other side, provided some quite general conditions are satisfied.

As suggested also in the discussion above (see also [24] and below), the paradigm of coherence seems to extend to large part, if not the whole of the universe $[62,54,55]$.

\section{Xiv. Topology of Quantum Spacetime}

The isomorphism between quantum coherence and fractals goes considerably deeper than we have presented so far. In a series of earlier papers (see [63][66]), MeN showed that $\mathcal{E}^{\infty}$ theory gives predictions of 
the mass spectrum of elementary particles and quarks in high energy physics based on the golden mean that are in remarkable agreement with those generally accepted experimentally and theoretically in the literature. Further, the same predictions are provided by a physical interpretation of four-dimensional fusion algebra, of Conne's non-commutative geometry and related theories such as Freedmann topological the-ory of four manifolds (wild topology), and Penrose space $X$ (of which $\mathcal{E}^{\infty}$ is a higher dimensional space). The fascinating details are beyond the scope of the present paper. We cite the results because they so strongly suggest that the mass spectrum based on the golden mean is a reflection of the quantum topology of spacetime [65].

This conjecture is reinforced, as MeN pointed out, in the simplest mechanical model for $\mathcal{E}^{\infty}$ theory, which is two golden mean coupled-oscillators, with frequencies of vibrations $\omega_{1}=\phi, \omega_{2}=1 / \phi$. That is because at least in the case of quarks, what we consider to be a particle can be thought of as a highly localized vibration, a standing wave simulating a particle (as in string theory).

Generalizing to $n$ such nested oscillators, Leila Marek-Crnjac was able to use Southwell and Dunkerley summation theorems for structural stability to obtain the masses of the elementary particles of the standard model, as well as the current quarks and constituent quarks [67]. The results are again very close to the theoretical and experimental masses found previously.

Marek-Crnjac also noted that the golden mean plays a central role in dynamic stability in KAM theory (see above), pointing out that string theory eliminates the wave-particle duality by using the highly localized vibration of a Planck length string that gives rise to a particle when perceived on lower energy levels [67]. In this way, the golden mean enters into the mass spectrum via the KAM theory. As it is the most irrational number (see sections 6 and 12) it plays the key role in the stability of periodic orbits and the onset of global chaos. The ratio of $\Omega / \omega$ (driver to oscillator frequency) is decisive as to whether the motion is localized (regular) or dissipative (stochastic). If the ratio is rational, the torus is destroyed. However, if it is irrational, the torus persists. A well-known application of this theory is the Kirkwood gaps - dips in the distribution of the main belt asteroids, which coincide with orbital resonances with Jupiter. What this means is that a particle is observed only when the energy is localized in a highly coherent vibration in an irrational frequency, and the most irrational frequency is $\phi$.

Marek-Crnjac's argument applies also to the circular thermodynamics of organisms and the fractal hierarchy of living activities developed in Sections 7-12. The mass spectrum of elementary particle and living activities are both a reflection of the universal quantum topology of spacetime.

\section{Coherent Brain Waves, Scale-Free}

\section{LAWS AND FRACTALS}

Laboratory observations on the brain have consistently detected spatially extended regions of coherent neuronal activities, most thoroughly described in recent years by Walter Freeman and colleagues [68],[69],[70]. These phenomena have been observed by Lashley since the 1940s, who introduced the notion of field. Karl Pribram proposed his holographic hypothesis of memory formation in the early 1960s that introduced the notion of coherence in analogy to laser theory [58]. Hiroomi Umezawa proposed the first quantum field theory of memory in 1967 (see [71],[72]), that includes both the notions of field and coherence.

Observations clearly show that the coherent cortical activities cannot be fully accounted for by the electric field of the extracellular dendritic current or the extracellular magnetic field from the high-density electric current inside the dendritic shafts, nor by chemical diffusion. Spatially extended patterns of phase-locked oscillations are intermittently present in resting, awake subjects, and in the same subjects actively engaged in cognitive tasks. They are best described as properties of the brain modulated upon engagement with the environment. These packets of waves extend over spatial domains covering much of the hemisphere in rabbits and cats [73]-[76] and over regions of $19 \mathrm{~cm}$ linear dimension in the human cortex with near zero phase dispersion [68], [77]-[79].

Umezawa's many-body model [80] is based on the quantum field theoretic notion of spontaneous breakdown of symmetry [81],[61], which requires the existence of Nambu-Goldstone (NG) massless particles. Examples are the phonon modes in crystals, the magnon modes in ferromagnets, etc. They can be described, as customary in quantum theory, as the quanta associated with certain waves such as the elastic wave in crystals, the spin wave in ferromagnets. The role of such waves is that of establishing long range correlation. The mechanism of spontaneous breakdown of symmetry generates the change of scale: from the microscopic scale of the elementary constituent dynamics to the macroscopic scale of the system observable ordered patterns. In ferromagnets, for example, the ordered patterns is described by magnetization, which for this reason is called the order parameter. The phase transition from zero magnetization to the magnetic phase is induced by some external weak magnetic field. The mathematical structure of the theory must be adequate to allow for physically distinct phases. Quantum field theory possesses such a mathematical structure. The ground states corresponding to physically distinct phases are characterized by distinct degrees of ordering described by different densities of NG modes condensed in them. Such a condensation of NG modes 
in the ground states is a coherent condensation, which physically expresses the in phase synchronized oscillations. In quantum mechanics, on the contrary, all the state representations are physically equivalent and therefore not useful to describe phase transitions [82].

The quantum variables relevant to the manybody model of the brain have been identified to be the electric dipole vibration modes of the water molecules that constitute the matrix in which neurons and glia cells are embedded [83],[84]. The spontaneous breakdown of the rotational symmetry of electrical dipoles of water and other molecules implies the existence of $\mathrm{NG}$ modes. These modes have been called the dipole wave quanta (DWQ). The system ground state is thus obtained in terms of coherent condensation of the DWQ, which are the agents responsible for neuronal coordination [83]-[85]. In particular, it is found that the memory state is a squeezed coherent state for the basic quantum variables expressed mesoscopically as amplitude and phase modulation of the carrier signal observed in electroencephalograms (EEGs) and electrocorticograms (ECoGs).

Laboratory observations show that self-similarity characterizes the brain ground state. Measurements of the durations, recurrence intervals and diameters of neocortical EEG phase patterns have power-law distribution with no detectable minima. The power spectral densities in both time and space conform to power law distribution [73],[74], a hallmark of fractals. It is therefore consistent with the squeezed coherent state for memory found earlier.

Coherent signals are obtained when the electrodes are closely spaced. A lot of data have been collected by Freeman's group on ECoG spatial imaging coming from small, high-density electrode arrays fixed on the surfaces of the olfactory, visual, auditory, or somatic modules. Spectral analysis of the ECoG shows broad distribution of the frequency components, where the temporal power spectral density in log-log coordinates is power-law, $1 / f^{\alpha}$, in segments. Below an inflection in the theta-alpha range the power spectral density is at, $\alpha=0$. Above, the $\log _{10}$ power decreases linearly with increasing $\log 10$ frequency in the betagamma range $(12.5-80 \mathrm{~Hz})$ with the exponent $\alpha$ between 2 and 3 . One can show that in slow-wave sleep, the exponent averages near 3 ; in seizures it averages near 4 . On the basis of what was said above, such values of the slope $\alpha$ provide corresponding values of the fractal dimension $d \equiv \alpha$, with deformation parameter $q \equiv 1 / f^{\alpha}$ and coherent strength corresponding to the power spectral density. Brief epochs of narrow band oscillation create multiple peaks, indicating a departure from the scale free regime (the straight line) and therefore the presence of structures emergent from the background activity.

The observed power law signals the scale free ("spatial similarity") property of the brain ground states.
The spatial similarities reveal the long spatial distances across which synaptic interactions can sustain coherence, namely the high-density coordination of neuron firing by synaptic interaction, in agreement with the dissipative model prediction.

A confirmation of the brain scale-free behaviour comes also from the group of Dietmar Plenz at the US National Institute of Mental Health. They have identified neuronal avalanches - cascades of neuronal activities that follow precise $1 / f$ power laws - in the excitatory neurons of the superficial layers in isolated neocortex preparations in vitro as well as in awake animals and humans in vivo [86]. They showed that the neuronal avalanche of the default state with the $1 / f$ signature of self-organized criticality gives the optimum response to inputs as well as maximum information capacity (reviewed elsewhere in more detail by MWH [87]).

Most significantly, the avalanche dynamics give rise to coherence potentials consisting of subsets of avalanches in which the precise waveform of the local field potential is replicated with high fidelity in distant network sites. The process is independent of spatial distance and includes near instantaneous neuronal activities as well as sequential activities over many time scales.

Most coherence potentials are spatially disjunct. Local Field Potentials (LFPs) of successive coherence potentials are not similar, but they are practically identical within a coherence potential among all the participating sites, there being no growth or dissipation during propagation. This suggests that the waveform of a coherence potential is a high-dimensional coding space in information processing of the brain. For decades, phase-locked neuronal activity has been reliably recorded using the LFPs or EEG and was found to correlate with the presentation of stimulus in animals and visual perception in humans.

\section{Golden Music of the Brain}

In living systems, fractals must satisfy the state of quantum coherence which maximizes global cohesion as well as local autonomy, and enables energy from any local level to spread to the global and conversely concentrate energy to any domain from the entire system. Our discussion suggests that fractals with fractal dimension in the golden mean (golden fractals) might be the most effective in giving autonomy to the greatest number of cycles. On the other hand, global cohesion is also ensured, because cycles in a fractal hierarchy are quantum coherent; energy can be shared between global and local. The golden mean is also close to an infinite number of rational ratios, so special resonances or correlations can be easily established. Thus, one would expect biological rhythms in general to conform to the golden mean, although no such survey has yet been carried out. The golden mean figures 
prominently in the EEG frequencies of the resting brain (see Table 1). Belinda Pletzer, Hubert Kerschbaum and Wolfgang Klimesch proposed that brain frequencies that never synchronize in the resting brain may play an important role in the organization of groups of cells in keeping their rhythms distinct and free from mutual interference. They suggested that this can be achieved via frequencies in irrational multiples in the resting (default) brain [88] (see also [87]). Table 1 lists the typical EEG frequency bands.

Table 1 : Typical EEG frequency bands and subbands and corresponding periods (from [88]).

\begin{tabular}{llcccc}
\hline Band & $(\mathrm{Hz})$ & Subband & $(\mathrm{Hz})$ & Peak $(\mathrm{Hz})$ & Period $(\mathrm{ms})$ \\
\hline delta & \multirow{2}{*}{$1.5-4$} & delta 1 & $1-2$ & 1.5 & 667 \\
& & delta2 & $2-3$ & 2.5 & 400 \\
theta & $4-10$ & theta1 & $3-5$ & 4 & 250 \\
& & theta2 & $5-8$ & 6.5 & 154 \\
alpha & \multirow{2}{*}{$8-12$} & alpha & $8-12$ & 10 & 100 \\
beta & \multirow{2}{*}{$10-30$} & beta1 & $12-20$ & 16 & 62.5 \\
& & beta2 & $20-30$ & 25 & 40 \\
gamma & \multirow{2}{*}{$30-80$} & gamma1 & $30-50$ & 40 & 25 \\
& & gamma2 & $50-80$ & 65 & 15 \\
fast & \multirow{2}{*}{$80-200$} & ripples1 & $80-120$ & 100 & 10 \\
ripples & & ripples2 & $120-200$ & 160 & 6.25 \\
\hline
\end{tabular}

The classical EEG frequency bands can indeed be described as a geometric series with a ratio between neighbouring frequencies approximating $\Phi=1.618$, and the successive frequencies are the sum of two previous ones, as in the Fibonacci sequence. Intuitively at least, one can see that the golden mean provides the highest physiologically possible desynchronized frequencies, and at the same time, the potential for spontaneous diverse coupling and uncoupling between rhythms and a rapid transition from resting state to activity.

A team of researchers led by Miles Whittington have been studying the intricacies in the golden music of the brain by recording from multiple layers of the neocortex simultaneously. They found multiple local neuron assemblies supporting different discrete frequencies in the neocortex network, and the relationships between different frequencies appear designed to minimize interference and to allow diverse coupling of activities via stable phase interactions and the control of the amplitude of one frequency in relation to the phase of another [89].

The $1 / f$ pattern of EEG is really a timeaveraged smoothed collection of multiple, discrete frequencies, and does not represent all the frequencies and combination of frequencies present in the brain. (It is like a recording of a Mozart symphony that averages out all the sounds made in discrete periods of time, so the music is completely buried.) Detailed observations made by the team have shown that at least three discrete frequencies $\delta(1-3 \mathrm{~Hz}), \theta(6-9 \mathrm{~Hz})$, and $\gamma(30-50$ $\mathrm{Hz}$ ) are often expressed simultaneously, and can be associated with further much slower rhythms both in vivo and in vitro.
Discrete frequencies ranging from low $\delta$ to high can $\gamma$ be produced from a single area of the isolated neocortex in vitro, with peak frequencies distributed according to the golden mean.

To keep simultaneously occurring frequencies apart and minimize interferences, the solution is indeed to have ratios of frequencies that are irrational numbers. Coexistent $\gamma_{1}$ and $\beta_{2}$ rhythms in the cortex, for example, are generated in two different layers and survive physical separation of the layers. The ratio of peak frequencies is approximately $\Phi$, resulting in a periodic pattern of change in low-level synchrony between the layers with a period equal to the sum of the two periods of oscillation present. This phenomenon can occur to some extent with any pair of co-expressed frequencies. But using $\Phi$ as a common ratio between adjacent frequencies in the EEG spectrum enables the neocortex to pack the available frequency space (thereby maximising the information processing capacity, or the capacity to produce the most music). If the cortex uses different frequency bands to process different aspects of incoming information, then it must also have the ability to combine information held in these bands to reconstruct the input; hence the importance of keeping them separate, as the golden mean does.

It is also possible for a local neuron assembly generating a single frequency rhythm to switch frequencies. Such changes are facilitated by a range of mechanisms including changes in neuronal intrinsic conductances and non-reciprocal interactions with other regions oscillating at a similar frequency. After stimulation, $\gamma$ frequencies can transform to $\beta$ frequencies 
(approximately halved) due to inhibitory postsynaptic potentials on the principal cells generating the action potentials.

Can interactions between multiple spatiotemporal scales of activity tell us anything about how the cortex processes sensory information?

In the time domain, the ability to distinguish rapidly changing features of an input from more slowly changing features provides an efficient means of recognizing objects. Feature detection over a range of time scales can reproduce many properties of individual neurons in the visual cortex. Thus, from a computational perspective, it is an advantage for the cortex to process different temporal scales of information separately, using different frequencies. It has been shown that rhythms with larger temporal scales (slower frequencies) facilitate interactions over greater distances in cortical networks, i.e., they may synchronize over larger areas of the visual map in the retina of the eyes. Thus, different frequencies may have a role for processing sensory information on different spatial scales. In a visual task designed to test perception shifting from features of an object with low spatial frequency to those with high spatial frequency, a direct correlation was found between the spatial scale of the sensory object and the temporal scale (frequency) of associated cortical rhythms. Thus cross-frequency phase synchronization is a possible means of combining information from different frequency channels to fully represent a sensory object.

\section{XVil. Conclusion}

We have considered the fabric of spacetime from the widest perspectives, drawing on findings from mathematics, quantum physics, far from equilibrium thermodynamics, biology, and neurobiology. Although a definite answer cannot be given to the question asked in the title of this paper, the totality of findings obtained by different authors discussed in previous Sections seem to converge and justify our speculation that spacetime is fractal and quantum coherent in the golden mean. Mathematically, the fractal universe is non-differentiable and discontinuous, yet dense in infinite dimensional spacetime. Physically, it is a quantum coherent universe consisting of an infinite diversity of autonomous agents all participating in co-creating organic, fractal spacetime by their multitudinous coupled activity cycles. Biologically, this fractal coherent space-time could also provide the fabric of conscious awareness mirrored in the quantum coherence of our brain states. This view depicts a new organic cosmogony consonant with that of Alfred North Whitehead [1], resolving major paradoxes associated with the classical mechanics, and paving the way to reconciling or transcending quantum theory and general relativity. Much work remains to be done in order to provide a definitive answer to our question of whether fractal self-similarity, golden ratio and coherence are characterizing features of the fabric of spacetime.

As this manuscript is going to press, the first ever observation is reported that the brightness of some stars pulsate at primary and secondary frequencies whose ratios are near the golden mean [John $F$. Lindner, Vivek Kohar, Behnam Kia, Michael Hippke, John G. Learned, and William L. Ditto, Strange Nonchaotic Stars, Phys. Rev. Lett. 114, 054101 (2015). arxiv.org/abs/1501.01747]. This evidence of strange nonchaotic dynamics recorded by the Kepler space telescope lends support to our vision of the fractal golden mean structure of spacetime.

\section{XViII. ACKNOWLedgment}

We thank Peter Saunders for stimulating and helpful discussions and for critical reading of successive drafts of this manuscript.

\section{Appendix 1. Zeno's Paradox}

Achilles is running a race with the tortoise. Achilles gives the tortoise a head start of 100 metres, say. If we suppose that both run at constant speed Achilles very fast and the tortoise very slow - then after some time, Achilles will have run 100 metres, bringing him to the tortoise's starting point. But during that time, the tortoise has run a much shorter distance, say 10 metres. It will then take Achilles some further time to run that distance, by which time the tortoise will have gone ahead farther; and then he would need more time still to reach that third point, while the tortoise moves ahead, and so on. Thus, whenever Achilles reaches somewhere the tortoise has been, he still has farther to go. Therefore, because there are an infinite number of points where the tortoise has already been for Achilles to reach, he can never overtake the tortoise [4].

\section{Appendix 2. Some Informal Definitions}

We provide here informal definitions (from [8]) of some mathematical terms that will be used in this paper; some, like deterministic chaos, have no generally agreed definition.

Set theory is the branch of mathematical logic about collections of mathematical objects. The modern study of set theory was initiated by German mathematicians Georg Cantor (1845-1918) and Richard Dedekind (1831-1916) in the 1870s. A closed set contains its own boundary, its complement is an open set which is a set that does not contain its boundary.

A Borel set is any set in a topological space that can be formed from open sets (or equivalently from closed sets) through the operations of countable union, countable intersection, and relative complement. 
A countable set is a set with the same number of elements as some subset of the set of natural numbers. The elements of a countable set can be counted one at a time, and although the counting may never finish, every element of the set will eventually be associated with a natural number.

The union of two sets $A$ and $B$ is the the set of all elements that belong to either set and is denoted by $A \cup B$. The intersection of $A$ and $B$ is the set of all elements that belong to both sets and is denoted by $A \cap B$. The relative complement of $A$ in $B$ is the set of elements that are in $B$ but are not in $A$.

A bijection is a mapping from a set $A$ to a set $B$ that is both an injection (one-to-one) and a surjection (onto); it relates each member of $A$ (the domain) to a unique member of $B$ (the range). Each element of $B$ also has a unique corresponding element in $A$.

The classical triadic Cantor set is obtained by dividing the unit line into three equal parts, discarding the middle part except for its end points, and repeating the operation with the two remaining parts ad infinitum. In the random version, it could be any of the three parts that is discarded at random after each division. A topological space is a set of points and a set of neighbourhoods for each point that satisfy a set of axioms relating to points and neighbourhoods. The definition of a topological space relies only on set theory and is the most general notion of a mathematical space.

For our purposes, the topological dimension is what one ordinarily understands by the concept of dimension. A point has topological dimension 0 , a curve has topological dimension 1 (whether it is closed up into a circle or not); a at sheet has a topological dimension of 2, as do the surface of a cylinder, sphere or doughnut, and so on.

The Menger-Urysohn dimension is a generalized topological dimension of topological spaces, arrived at by mathematical induction. It is based on the observation that, in n-dimensional Euclidean space $R^{n}$, the boundaries of $n$-dimensional balls have dimension $n-1$. Therefore it should be possible to define the dimension of a space inductively in terms of the dimensions of the boundaries of suitable open sets.

The Hausdorff dimension generalizes the notion of dimension to irregular sets such as fractals. For example, a Cantor set has a Hausdorff dimension of $\log 2 / \log 3$, the ratio of the logarithm to the base 2 of the parts remaining to the whole after each iteration.

A fractal is a mathematical set that typically displays self-similar patterns, and has dimensions that are fractions rather than integers. Geometric examples are branching trees, blood vessels, frond leaves etc.

Deterministic Chaos describes dynamical systems with unpredictable behavior that is highly sensitive to initial conditions, but nevertheless globally determined in the sense that the trajectories are confined within a region of phase space called strange attractors.

\section{References Références Referencias}

1. Ho MW. Golden geometry of E-infinity fractal spacetime. Story of phipart 5. Science in Society 2014, 62, 36-39.

2. Whitehead AN. Science and the Modern World, Lowell Lectures 1925, Collins Fontana Books, Glasgow, 1975.

3. Penrose R. The Road to Reality, A Complete Guide to the Laws of the Universe, Vintage Books, London, 2005. 1099 pp.

4. Wikipedia. Zeno's paradoxes. 20 October 2014.

5. Bergson H. Time and Free Will, An Essay on the Immediate Data of Consciousness (FL Pogson trans.), George Allen \& Unwin, Ltd., New York, 1916.

6. Whitehead, AN. Concept of Nature, Tarner Lectures delievered in Trinity College, Cambridge, November 1919, Gutenberg eBook, 16 July 2006.

7. Cantor G. Über unendliche, lineare Puntkmannigfaltigkeiten V. Mathematische Annelen 21, 545591; cited in [8].

8. Rosser JB. Complex Evolutionary Dynamics in Urban-Regional and Ecologi-Economic Systems, DOI 10.1007/978-1-4419-8828-7, Springer Science +Business Media, LLC 2011.

9. Mandelbrot BB. The Fractal Geometry of Nature, W.H. Freeman, San Francisco, 1983.

10. Lorenz E. N. Deterministic non-periodic ow. J. Atmos Sci. 1963, 20, 130-141.

11. Lorenz E. N. The Essence of Chaos, University of Washington Press, Seattle, 1993.

12. Ord, GN. Fractal space-time: a geometric analogue of relativistic quantum mechanics. J. Phys. A: Math. Gen. 1983, 16, 1869-84.

13. Feynman RP and Hibbs AR. Quantum Mechanics and Path Integrals, McGraw-Hill, New York, 1965.

14. Nottale L. Fractal Space-Time and Microphysics: Towards a Theory of Scale Relativity, World Scientific, 1993.

15. Nottale L. Scale Relativity and Fractal Space-Time, A New Approach in Unifying Relativity and Quantum Mechanics, Imperial College Press, London, 2011.

16. El Naschie MS. A review of E infinity theory and the mass spectrum of high energy particle physics. Chaos Solitons \& Fractals 2004,19, 209-36.

17. El Naschie MS. The theory of Cantorian spacetime and high energy particle physics (an informal review). Chaos, Solitons and Fractals 2009, 41, 2635-46.

18. Mauldin RD and Williams SC. Random recursive constructions: asymptotic geometric and topological properties. Trans. Amer. Math. Soc. 1986, 295, 325-46. 
19. Ho MW. The story of phi part 1. Science in Society 2014, 62, 24-26. www.academia.edu/7718556/The Story_of_Phi_Part_1_The_Mathematics

20. "There is something about phi, Chapter 9 - Fractals and the golden ratio" Javier Romanach, YouTube, accessed 1 March 2014, www.youtube.com/watch $? \mathrm{v}=\mathrm{BURjKRfOA9g}$

21. Ho MW. Watching the daisies grow. Science in Society 2014, 62, 27-29. www.academia.edu /7718580/Story_of_Phi_Part_2_Watching_the_Daisies Grow

22. Marek-Crnjac L. The Hausdorff Dimension of the Penrose universe. Phys. Res. Interna 2011, 874302, 4 pages

23. Connes A. Noncommutative Geometry, Paris, 1994, www.alainconnes.org/docs/book94bigpdf.pdf

24. Ho MW. The Rainbow and the Worm, the Physics of Organisms, World Scientific, 1993, 2nd edition, 1996, 3rd enlarged edition, 2008, Singapore and London. www.i-sis.org.uk/rnbwwrm.php

25. Schommers W. Space-time and quantum phenomena. In Quantum Theory and Pictures of Reality (W. Schommers ed.), pp. 217-77, SpringerVerlag, Berlin, 1989.

26. El Naschie MS. Quantum collapse of wave interference pattern in the two-slit experiment: a set theoretical resolution. Nonlinear Sci. Lett. A 2011, 2 , 1-8.

27. El Naschie MS. Topological-geometrical and physical interpretation of the dark energy of the cosmos as a halo energy of the Schrdinger quantum wave. J. Mod. Phys. 2013, 4, 591-6.

28. Ho MW. E infinity spacetime, quantum paradoxes and quantum gravity. Science in Society 2014, 62, 40-43. www.academia.edu/7718725/ E-infinity space_time_quantum_paradoxes_and_quantum gravity story_of phi part_6

29. "Our universe continually cycles through a series of 'aeons", The Daily Galaxy, 26 September 2011, www. dailygalaxy.com/my weblog/2011/09/we-cansee-through-the-big-bang-to-the-universe-that-existed-in-the-aeon-before- $h$ tml

30. Eugene WC. An introduction to KAM theory. Preprint January 2008, http://math.bu.edu/people/cew/ preprints/introkam.pdf

31. Ho MW. Golden cycles and organic spacetime. Science in Society 2014, 62, 32-34.

32. Kolmogorov-Arnold-Moser theorem. Wikipedia, 24 January 2014,

33. Effect of noise on the golden-mean quasiperiodicity at the chaos threshold. www.sgtnd.narod.ru/science /noise/2noise/eng/2noise.htm

34. Arnold tongue. Wikipedia, 4 February 2014. http://en.wikipedia.org/wiki/Arnold_tongue
35. Ivankov NY and Kuznetsov SP. Complex periodic orbits, renormalization, and scaling for quasiperiodic golden-mean transition to chaos. Phys. Rev. E2001, 63, 046210.

36. Lombardi OW and Lombardi MA. The golden mean in the solar system. The Fibonacci Quarterly 1984, 22, 70-75. www.fq.math.ca/22-1.html

37. Phi and the solar system. f Phi 1.618 The Golden Number, 13 May 2013, www.goldennumber.net /solar-system/

38. "Is the solar system stable?" Scott Tremaine, Institute for Advanced Study, Summer 2011, www.ias.edu/about/publications/ias-letter/articles/ 2011-summer/solar- system-tremaine

39. Sprott JC. Honors: A tribute to Dr Edward Norten Lorenz. EC Journal 2008, 55-61, http://sprott.physics.wisc.edu/lorenz.pdf

40. Sprott JC. Strange Attractors: Creating Patterns in Chaos, M\&T Books, New York, 1993.

41. Viswanath D. Symbolic dynamics and periodic orbits of the Lorenz at tractor. Nonlinearity 2003, 16, 1035-56.

42. Birman JS and Williams RF. Knotted periodic orbits in dynamical systems 1. Lorenz's equations. Topology 1983, 22, 47-82.

43. Gutzwiller M. Quantum chaos. Scientific American January 1992, republished 27 October 2008, www.scientificamerican.com/article/quantum-chaos -subatomic- worlds/

44. Selvam AM. Cantorian fractal space-time fluctuations in turbulent fluid flows and the kinetic theory of gases. Apeiron 2002, 9, 1-20.

45. Kraut DA, Carroll KS and Herschlag D. Challenges in enzyme mechanisms and energetics. Ann. Rev. Biochem. 2003, 72, 517-71.

46. Ho MW. Living Rainbow H2O, World Scientific and Imperial College Press, Singapore and London, 2012.

47. Ho MW. Water is the means, medium, and message of life. Int. J. Design, Nature and Ecodynamics 2014, 9, 1-12.

48. Ho MW. Illuminating water and life. Entropy 2014 , 16, 4874-91.

49. Ho MW. Circular thermodynamics of organisms and sustainable systems. Systems 2013, 1, 30-49.

50. Glauber RJ. Coherence and quantum detection. In Quantum Optics ( RJ Glauber ed.), Academic Press, New York, 1969.

51. Metabolic pathways. Sigma Aldrich, accessed 27 October 2014. www.sigmaaldrich.com/content /dam/sigma-aldrich/docs/Sigma/General_Information/metabolic pathways poster.pdf

52. Prigogine I. Time, structure and fluctuations. Nobel Lecture, 8 December 1977, www.nobelprize.org/ 
nobel prizes/chemistry/laureates/1977/prigoginelecture.pdf

53. G. Vitiello, Fractals as macroscopic manifestation of squeezed coherent states and brain dynamics. J. Physics: Conf Ser 2012, 380, 012021 (13 pp).

54. G. Vitiello, Fractals, coherent states and selfsimilarity induced noncommutative geometry. Phys. Lett. A376, 2527-2532 (2012).

55. G. Vitiello, On the isomorphism between dissipative systems, fractal selfsimilarity and electrodynamics. Toward an integrated vision of Nature. Systems 2 , 203-216 (2014).

56. H. O. Peitgen, H. Jürgens and D. Saupe, Chaos and Fractals. New frontiers of Science, (SpringerVerlag, Berlin 1986).

57. A. A. Andronov, A. A. Vitt, S. E. Khaikin, Theory of Oscillators, (Dover Publications, INC, N.Y. 1966).

58. E. Celeghini, M. Rasetti and G. Vitiello, Quantum dissipation. Ann. Phys. 215, 156-170 (1992).

59. G. 't Hooft, Quantum Gravity as a Dissipative Deterministic System. Classical and Quantum Gravity 16, 3263-3279 (1999);

60. M. Blasone, P. Jizba and G. Vitiello, Dissipation and quantization. Phys. Lett. A287, 205-210 (2001).

M. Blasone, E. Celeghini, P. Jizba and G. Vitiello, Quantization, group contraction and zero point energy. Phys. Lett. A310, 393-399 (2003).

M. Blasone, P. Jizba, F. Scardigli and G. Vitiello, Dissipation and quantization in composite systems. Phys. Lett. A373, 4106-4112 (2009).

61. M. Blasone, P. Jizba and G. Vitiello Quantum Field Theory and its Macroscopic Manifestations, (Imperial College Press, London 2011).

62. Ho MW. Quantum world coming series. Science in Society 2004, 22, 4-15

63. El Naschie MS. Quantum loops, wild topology and fat Cantor sets in transfinite high-energy physics. Chaos, Solitons \& Fractals 2002, 13, 1167-74.

64. El Naschie MS. Wild topology, hyperbolic geometry and fusion algebra in high-energy physics. Chaos, Solitons \& Fractals 2002, 13, 1935-45.

65. El Naschie MS. On the exact mass spectrum of quarks. Chaos Solitons \& Fractals 2002, 14, 369-76.

66. El Naschie MS. On a class of general theories for high energy particle physics. Chaos, Solitons \& Fractals 2002, 14, 649-68.

67. Marek-Crnjac L. The mass spectrum of high energy elementary particles via El Naschie's $\mathcal{E}^{\infty}$ golden mean nested oscillators, the Dunkerly-Southwell eigenvalue theorems and KAM. Chaos Solitons \& Fractals 2003, 18, 125-33.

68. Freeman WJ. Mass Action in the Nervous System, Academic Press, New York, 1975, 2004.

69. Freeman WJ. Neurodynamics, an Exploration of Mesoscopic Brain Dynamics, Springer- Verlag, 2000.
70. Freeman WJ. How Brains make up Their Minds, Columbia University Press, 2001.

71. Umezawa H. Advanced Field Theory: Micro, Macro and Thermal Physics, American Institute of Physics, New York, 1993.

72. Vitiello G. Hiroomi Umezawa and quantum field theory. NeuroQuantology 2011, 9, DOI: 10.14704/nq.2011.9.3.450

73. Freeman WJ. Origin, Structure, and role of background EEG activity. Part 1. Analytic phase. Clin Neurol. 2004, 115, 2077-88.

74. Freeman WJ. Origin, Structure, and role of background EEG activity. Part 2. Analytic amplitude. Clin Neurol. 2004, 115, 2089-107.

75. Freeman WJ. Origin, Structure, and role of background EEG activity. Part 3. Neural frame classification. Clin. Neurol. 2005, 116, 1118-29.

76. Freeman WJ. Phase transitions in the neuropercolation nodel of neural populations with mixed local and non-local interactions. Biol. Cybern. 2005, 92, 367-79.

77. Bassett DS, Meyer-Lindenberg A, Achard S, Duke T, Bullmore E. Adaptive reconfiguration of fractal small-world human brain functional networks. PNAS 2006, 103, 19518-23.

78. Freeman WJ and Burke BC. A neurobiological theory of meaning in perception. Part 4. Multicortical patterns of amplitude modulation in gamma EEG. Int J. Bifurc. \& Chaos 2003, 13, 2857-66.

79. Freeman WJ and Rogers LJ. A neurobiological theory of meaning in perception. Part 5. Multicortical patterns of phase modulation in gamma EEG. Int. J. Bifurc. \& Chaos 2003, 13, 2867-87.

80. Ricciardi LM and Umezawa $\mathrm{H}$. Brain and physics of many-body problems. Kybernetik 1967, 4, 44-48.

81. Vitiello G. My Double Unveiled, John Benjamin Pub. Co., Amsterdam, 2001.

82. Umezawa H and Vitiello G. Quantum Mechanics, Bibliopolis, Naples, 1985.

83. Vitiello G. Dissipation and memory capacity in the quantum brain model. Int. J. Mod. Phys. 1995, B9, 973.

84. Jibu M and Yasue K. Quantum Brain Dynamics and Consciousness, John Benjamin, Amsterdam, 1995.

85. Del Giudice E, Preparata G and Vitiello G. Water as a free electric dipole laser. Phys. Rev. Lett. 1988, 61, 1085-88.

86. Plenz D. Neuronal avalanches and coherence potentials. Eur. Phys. J. Special Topics 2012, 205, 259-301.

87. Ho MW. Golden music of the brain. Science in Society 2014, 62.

88. Pletzer $\mathrm{B}$, Kerschbaum $\mathrm{H}$ and Kliesch W. When frequencies never synchronize: the golden mean and the resting EEG. Brain Research 2010, 1335, 91-102. 
89. Roopun RK, Kramer MA, Carracedo LM, Kaiser M, Davies $\mathrm{CH}$, Traub RD, Kopell NJ and Whittingon MA. Temporal interactions between cortical rhythms. Frontiers in Neuroscience 2008, doi:10.3389/neuro.01.034.2008 
GlobAl JOURNALS INC. (US) GUIDELINES HANDBOOK 2015

WWW.GLOBALJOURNALS.ORG 


\section{FELLOWS}

\section{FELLOW OF ASSOCIATION OF RESEARCH SOCIETY IN SCIENCE (FARSS)}

Global Journals Incorporate (USA) is accredited by Open Association of Research Society (OARS), U.S.A and in turn, awards "FARSS" title to individuals. The 'FARSS' title is accorded to a selected professional after the approval of the Editor-inChief/Editorial Board Members/Dean.

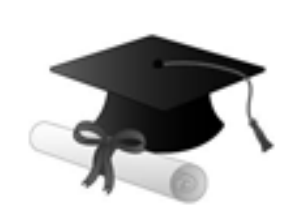

The "FARSS" is a dignified title which is accorded to a person's name viz. Dr. John E. Hall, Ph.D., FARSS or William Walldroff, M.S., FARSS.

FARSS accrediting is an honor. It authenticates your research activities. After recognition as FARSB, you can add 'FARSS' title with your name as you use this recognition as additional suffix to your status. This will definitely enhance and add more value and repute to your name. You may use it on your professional Counseling Materials such as CV, Resume, and Visiting Card etc.

The following benefits can be availed by you only for next three years from the date of certification:

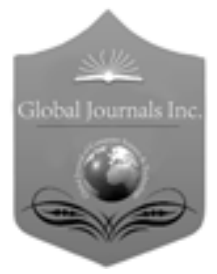

FARSS designated members are entitled to avail a $40 \%$ discount while publishing their research papers (of a single author) with Global Journals Incorporation (USA), if the same is accepted by Editorial Board/Peer Reviewers. If you are a main author or coauthor in case of multiple authors, you will be entitled to avail discount of $10 \%$.

Once FARSB title is accorded, the Fellow is authorized to organize a symposium/seminar/conference on behalf of Global Journal Incorporation (USA). The Fellow can also participate in conference/seminar/symposium organized by another institution as representative of Global Journal. In both the cases, it is mandatory for

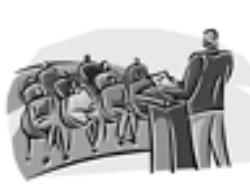
him to discuss with us and obtain our consent.

You may join as member of the Editorial Board of Global Journals Incorporation (USA) after successful completion of three years as Fellow and as Peer Reviewer. In addition, it is also desirable that you should organize seminar/symposium/conference at least once.

We shall provide you intimation regarding launching of e-version of journal of your stream time to time.This may be utilized in your library for the enrichment of knowledge of your students as well as it can also be helpful for the concerned faculty members.

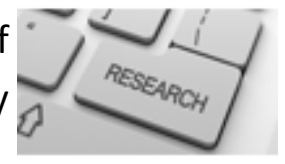

(C) Copyright by Global Journals Inc.(US) | Guidelines Handbook 
The FARSS can go through standards of OARS. You can also play vital role if you have any suggestions so that proper amendment can take place to improve the same for the Journals Rescarch benefit of entire research community.

As FARSS, you will be given a renowned, secure and free professional email address with 100 GB of space e.g. johnhall@globaljournals.org. This will include Webmail,

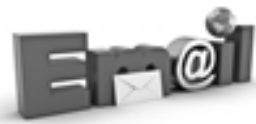
Spam Assassin, Email Forwarders,Auto-Responders, Email Delivery Route tracing, etc.

The FARSS will be eligible for a free application of standardization of their researches.

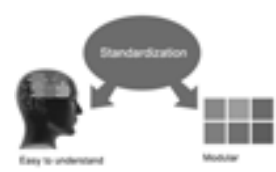
Standardization of research will be subject to acceptability within stipulated norms as the next step after publishing in a journal. We shall depute a team of specialized research professionals who will render their services for elevating your researches to next higher level, which is worldwide open standardization.

The FARSS member can apply for grading and certification of standards of their educational and Institutional Degrees to Open Association of Research, Society U.S.A. Once you are designated as FARSS, you may send us a scanned copy of all of your credentials. OARS will verify, grade and certify them. This will be based on your academic records, quality of research papers published by you, and some more criteria. After certification of all your credentials by OARS, they will be published on

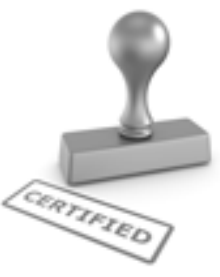
your Fellow Profile link on website https://associationofresearch.org which will be helpful to upgrade the dignity.

The FARSS members can avail the benefits of free research podcasting in Global Research Radio with their research documents. After publishing the work, (including

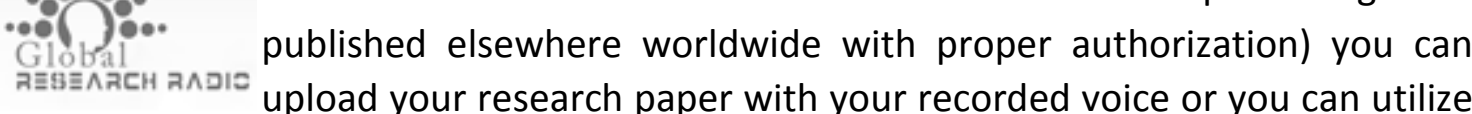

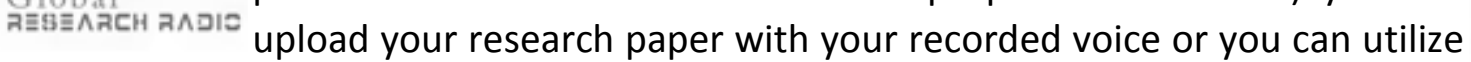
chargeable services of our professional RJs to record your paper in their voice on request.

The FARSS member also entitled to get the benefits of free research podcasting of

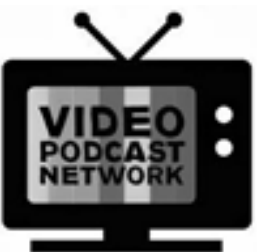
their research documents through video clips. We can also streamline your conference videos and display your slides/ online slides and online research video clips at reasonable charges, on request.

(C) Copyright by Global Journals Inc.(US) | Guidelines Handbook 


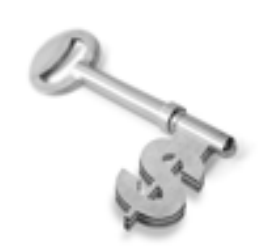

The FARSS is eligible to earn from sales proceeds of his/her researches/reference/review Books or literature, while publishing with Global Journals. The FARSS can decide whether he/she would like to publish his/her research in a closed manner. In this case, whenever readers purchase that individual research paper for reading, maximum $60 \%$ of its profit earned as royalty by Global Journals, will be credited to his/her bank account. The entire entitled amount will be credited to his/her bank account exceeding limit of minimum fixed balance. There is no minimum time limit for collection. The FARSS member can decide its price and we can help in making the right decision.

The FARSS member is eligible to join as a paid peer reviewer at Global Journals Incorporation (USA) and can get remuneration of $15 \%$ of author fees, taken from the author of a respective paper. After reviewing 5 or more papers you can request to transfer the amount to your bank account.

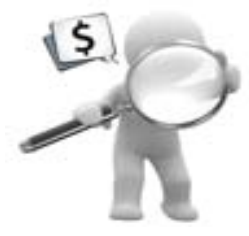

\section{MEMBER OF ASSOCIATION OF RESEARCH SOCIETY IN SCIENCE (MARSS)}

The ' MARSS ' title is accorded to a selected professional after the approval of the Editor-in-Chief / Editorial Board Members/Dean.

The "MARSS" is a dignified ornament which is accorded to a person's name viz. Dr. John E. Hall, Ph.D., MARSS or William Walldroff, M.S., MARSS.

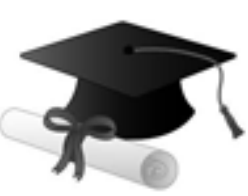

MARSS accrediting is an honor. It authenticates your research activities. After becoming MARSS, you can add 'MARSS' title with your name as you use this recognition as additional suffix to your status. This will definitely enhance and add more value and repute to your name. You may use it on your professional Counseling Materials such as CV, Resume, Visiting Card and Name Plate etc.

The following benefitscan be availed by you only for next three years from the date of certification.

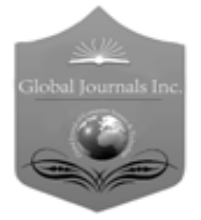

MARSS designated members are entitled to avail a $25 \%$ discount while publishing their research papers (of a single author) in Global Journals Inc., if the same is accepted by our Editorial Board and Peer Reviewers. If you are a main author or coauthor of a group of authors, you will get discount of $10 \%$.

As MARSS, you will be given a renowned, secure and free professional email address with 30 GB of space e.g. johnhall@globaljournals.org. This will include Webmail, Spam Assassin, Email Forwarders,Auto-Responders, Email Delivery Route tracing, etc.

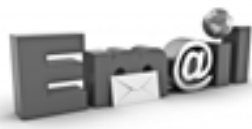

(C) Copyright by Global Journals Inc.(US) | Guidelines Handbook 


\section{We shall provide you intimation regarding launching of e-version of journal of your}

$\underbrace{\sim}_{0}$ stream time to time.This may be utilized in your library for the enrichment of knowledge of your students as well as it can also be helpful for the concerned faculty members.

The MARSS member can apply for approval, grading and certification of standards of their educational and Institutional Degrees to Open Association of Research, Society U.S.A.

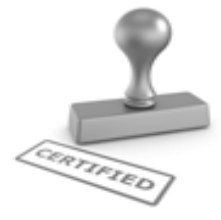

Once you are designated as MARSS, you may send us a scanned copy of all of your credentials. OARS will verify, grade and certify them. This will be based on your academic records, quality of research papers published by you, and some more criteria.

It is mandatory to read all terms and conditions carefully. 


\section{AUXILIARY MEMBERSHIPS}

\section{Institutional Fellow of Global Journals Incorporation (USA)-OARS (USA)}

Global Journals Incorporation (USA) is accredited by Open Association of Research Society, U.S.A (OARS) and in turn, affiliates research institutions as "Institutional Fellow of Open Association of Research Society" (IFOARS).

The "FARSC" is a dignified title which is accorded to a person's name viz. Dr. John E. Hall, Ph.D., FARSC or William Walldroff, M.S., FARSC.

The IFOARS institution is entitled to form a Board comprised of one Chairperson and three to five board members preferably from different streams. The Board will be recognized as "Institutional Board of Open Association of Research Society"-(IBOARS).

The Institute will be entitled to following benefits:

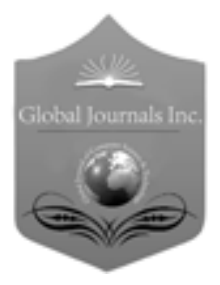

The IBOARS can initially review research papers of their institute and recommend them to publish with respective journal of Global Journals. It can also review the papers of other institutions after obtaining our consent. The second review will be done by peer reviewer of Global Journals Incorporation (USA) The Board is at liberty to appoint a peer reviewer with the approval of chairperson after consulting us.

The author fees of such paper may be waived off up to $40 \%$.

The Global Journals Incorporation (USA) at its discretion can also refer double blind peer reviewed paper at their end to the board for the verification and to get recommendation for final stage of acceptance of publication.

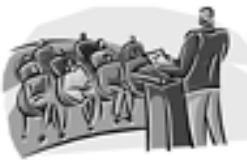

The IBOARS can organize symposium/seminar/conference in their counu y un vcran u Global Journals Incorporation (USA)-OARS (USA). The terms and conditions can be discussed separately.

The Board can also play vital role by exploring and giving valuable suggestions regarding the Standards of "Open Association of Research Society, U.S.A (OARS)" so that proper amendment can take place for the benefit of entire research community. We shall provide details of particular standard only on receipt of request from the Board.

The board members can also join us as Individual Fellow with $40 \%$ discount on total fees applicable to Individual Fellow. They will be entitled to avail all the benefits as declared. Please visit Individual Fellow-sub menu of GlobalJournals.org to have more relevant details. 
We shall provide you intimation regarding launching of e-version of journal of your stream time to time. This may be utilized in your library for the enrichment of knowledge of your students as well as it can also be helpful for the concerned faculty members.

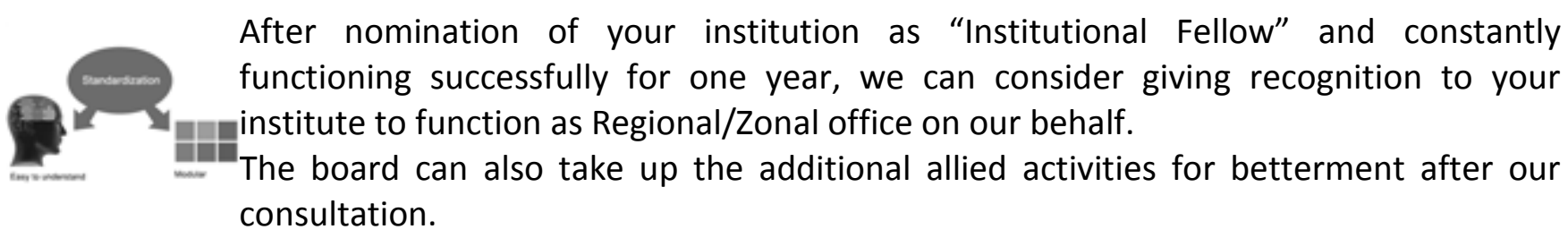

The following entitlements are applicable to individual Fellows:

Open Association of Research Society, U.S.A (OARS) By-laws states that an individual Fellow may use the designations as applicable, or the corresponding initials. The Credentials of individual Fellow and Associate designations signify that the individual has gained knowledge of the fundamental concepts. One is magnanimous and proficient in an expertise course covering the professional code of conduct, and follows recognized standards of practice.
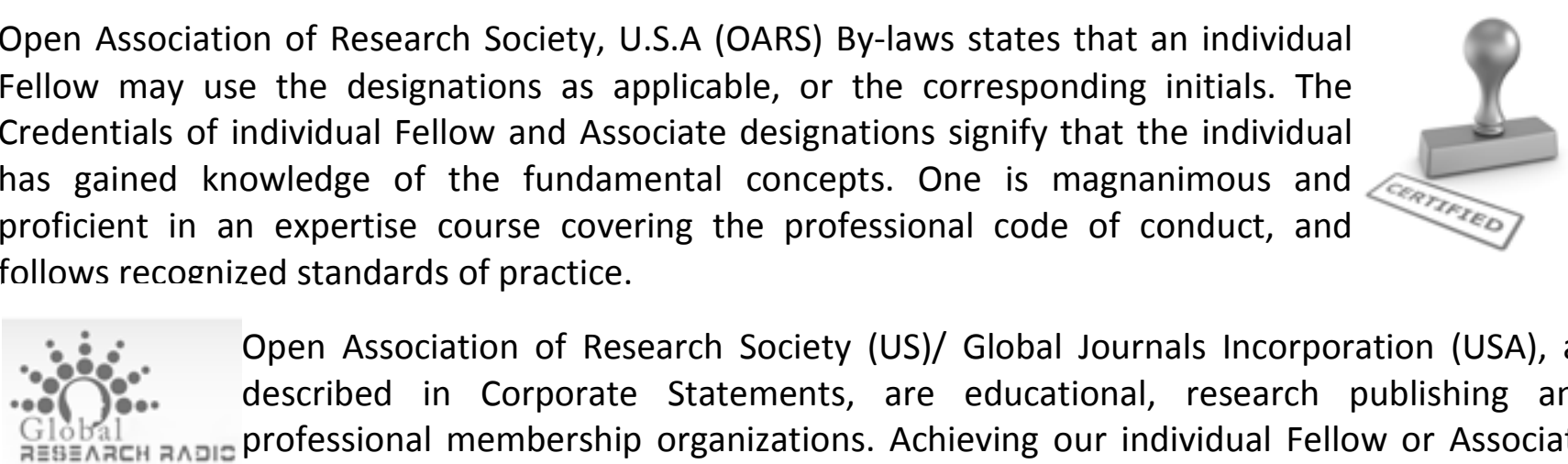

Open Association of Research Society (US)/ Global Journals Incorporation (USA), as described in Corporate Statements, are educational, research publishing and professional membership organizations. Achieving our individual Fellow or Associate status is based mainly on meeting stated educational research requirements.

Disbursement of $40 \%$ Royalty earned through Global Journals : Researcher $=50 \%$, Peer Reviewer $=37.50 \%$, Institution $=12.50 \%$ E.g. Out of $40 \%$, the $20 \%$ benefit should be passed on to researcher, $15 \%$ benefit towards remuneration should be given to a reviewer and remaining $5 \%$ is to be retained by the institution.

We shall provide print version of 12 issues of any three journals [as per your requirement] out of our 38 journals worth $\$ 2376$ USD.

\section{Other:}

The individual Fellow and Associate designations accredited by Open Association of Research Society (US) credentials signify guarantees following achievements:

> The professional accredited with Fellow honor, is entitled to various benefits viz. name, fame, honor, regular flow of income, secured bright future, social status etc.

(C) Copyright by Global Journals Inc.(US) | Guidelines Handbook 
$>$ In addition to above, if one is single author, then entitled to $40 \%$ discount on publishing research paper and can get $10 \%$ discount if one is co-author or main author among group of authors.

> The Fellow can organize symposium/seminar/conference on behalf of Global Journals Incorporation (USA) and he/she can also attend the same organized by other institutes on behalf of Global Journals.

$>$ The Fellow can become member of Editorial Board Member after completing 3yrs.

$>$ The Fellow can earn $60 \%$ of sales proceeds from the sale of reference/review books/literature/publishing of research paper.

$>$ Fellow can also join as paid peer reviewer and earn 15\% remuneration of author charges and can also get an opportunity to join as member of the Editorial Board of Global Journals Incorporation (USA)

- This individual has learned the basic methods of applying those concepts and techniques to common challenging situations. This individual has further demonstrated an in-depth understanding of the application of suitable techniques to a particular area of research practice.

\section{Note :}

IV.

$>$ In future, if the board feels the necessity to change any board member, the same can be done with the consent of the chairperson along with anyone board member without our approval.

$>$ In case, the chairperson needs to be replaced then consent of $2 / 3$ rd board members are required and they are also required to jointly pass the resolution copy of which should be sent to us. In such case, it will be compulsory to obtain our approval before replacement.

$>$ In case of "Difference of Opinion [if any]" among the Board members, our decision will be final and binding to everyone. 


\section{PROCESS OF SUBMISSION OF RESEARCH PAPER}

The Area or field of specialization may or may not be of any category as mentioned in 'Scope of Journal' menu of the GlobalJournals.org website. There are 37 Research Journal categorized with Six parental Journals GJCST, GJMR, GJRE, GJMBR, GJSFR, GJHSS. For Authors should prefer the mentioned categories. There are three widely used systems UDC, DDC and LCC. The details are available as 'Knowledge Abstract' at Home page. The major advantage of this coding is that, the research work will be exposed to and shared with all over the world as we are being abstracted and indexed worldwide.

The paper should be in proper format. The format can be downloaded from first page of 'Author Guideline' Menu. The Author is expected to follow the general rules as mentioned in this menu. The paper should be written in MS-Word Format (*.DOC, ${ }^{*}$.DOCX).

The Author can submit the paper either online or offline. The authors should prefer online submission. Online Submission: There are three ways to submit your paper:

(A) (I) First, register yourself using top right corner of Home page then Login. If you are already registered, then login using your username and password.

(II) Choose corresponding Journal.

(III) Click 'Submit Manuscript'. Fill required information and Upload the paper.

(B) If you are using Internet Explorer, then Direct Submission through Homepage is also available.

(C) If these two are not conveninet, and then email the paper directly to dean@globaljournals.org.

Offline Submission: Author can send the typed form of paper by Post. However, online submission should be preferred.

(C) Copyright by Global Journals Inc.(US) | Guidelines Handbook 


\section{PREFERRED AUTHOR GUIDELINES}

\section{MANUSCRIPT STYLE INSTRUCTION (Must be strictly followed)}

Page Size: $8.27^{\prime \prime}$ X $11^{\prime \prime \prime}$

- Left Margin: 0.65

- $\quad$ Right Margin: 0.65

- Top Margin: 0.75

- Bottom Margin: 0.75

- $\quad$ Font type of all text should be Swis 721 Lt BT.

- Paper Title should be of Font Size 24 with one Column section.

- Author Name in Font Size of 11 with one column as of Title.

- Abstract Font size of 9 Bold, "Abstract" word in Italic Bold.

- Main Text: Font size 10 with justified two columns section

- $\quad$ Two Column with Equal Column with of 3.38 and Gaping of .2

- $\quad$ First Character must be three lines Drop capped.

- $\quad$ Paragraph before Spacing of $1 \mathrm{pt}$ and After of $0 \mathrm{pt}$.

- $\quad$ Line Spacing of $1 \mathrm{pt}$

- Large Images must be in One Column

- Numbering of First Main Headings (Heading 1) must be in Roman Letters, Capital Letter, and Font Size of 10.

- Numbering of Second Main Headings (Heading 2) must be in Alphabets, Italic, and Font Size of 10.

You can use your own standard format also.

Author Guidelines:

1. General,

2. Ethical Guidelines,

3. Submission of Manuscripts,

4. Manuscript's Category,

5. Structure and Format of Manuscript,

6. After Acceptance.

\section{GENERAL}

Before submitting your research paper, one is advised to go through the details as mentioned in following heads. It will be beneficial, while peer reviewer justify your paper for publication.

Scope

The Global Journals Inc. (US) welcome the submission of original paper, review paper, survey article relevant to the all the streams of Philosophy and knowledge. The Global Journals Inc. (US) is parental platform for Global Journal of Computer Science and Technology, Researches in Engineering, Medical Research, Science Frontier Research, Human Social Science, Management, and Business organization. The choice of specific field can be done otherwise as following in Abstracting and Indexing Page on this Website. As the all Global 
Journals Inc. (US) are being abstracted and indexed (in process) by most of the reputed organizations. Topics of only narrow interest will not be accepted unless they have wider potential or consequences.

\section{ETHICAL GUIDELINES}

Authors should follow the ethical guidelines as mentioned below for publication of research paper and research activities.

Papers are accepted on strict understanding that the material in whole or in part has not been, nor is being, considered for publication elsewhere. If the paper once accepted by Global Journals Inc. (US) and Editorial Board, will become the copyright of the Global Journals Inc. (US).

Authorship: The authors and coauthors should have active contribution to conception design, analysis and interpretation of findings. They should critically review the contents and drafting of the paper. All should approve the final version of the paper before submission

The Global Journals Inc. (US) follows the definition of authorship set up by the Global Academy of Research and Development. According to the Global Academy of R\&D authorship, criteria must be based on:

1) Substantial contributions to conception and acquisition of data, analysis and interpretation of the findings.

2) Drafting the paper and revising it critically regarding important academic content.

3) Final approval of the version of the paper to be published.

All authors should have been credited according to their appropriate contribution in research activity and preparing paper. Contributors who do not match the criteria as authors may be mentioned under Acknowledgement.

Acknowledgements: Contributors to the research other than authors credited should be mentioned under acknowledgement. The specifications of the source of funding for the research if appropriate can be included. Suppliers of resources may be mentioned along with address.

Appeal of Decision: The Editorial Board's decision on publication of the paper is final and cannot be appealed elsewhere.

Permissions: It is the author's responsibility to have prior permission if all or parts of earlier published illustrations are used in this paper.

Please mention proper reference and appropriate acknowledgements wherever expected.

If all or parts of previously published illustrations are used, permission must be taken from the copyright holder concerned. It is the author's responsibility to take these in writing.

Approval for reproduction/modification of any information (including figures and tables) published elsewhere must be obtained by the authors/copyright holders before submission of the manuscript. Contributors (Authors) are responsible for any copyright fee involved.

\section{SUBMISSION OF MANUSCRIPTS}

Manuscripts should be uploaded via this online submission page. The online submission is most efficient method for submission of papers, as it enables rapid distribution of manuscripts and consequently speeds up the review procedure. It also enables authors to know the status of their own manuscripts by emailing us. Complete instructions for submitting a paper is available below.

Manuscript submission is a systematic procedure and little preparation is required beyond having all parts of your manuscript in a given format and a computer with an Internet connection and a Web browser. Full help and instructions are provided on-screen. As an author, you will be prompted for login and manuscript details as Field of Paper and then to upload your manuscript file(s) according to the instructions. 
To avoid postal delays, all transaction is preferred by e-mail. A finished manuscript submission is confirmed by e-mail immediately and your paper enters the editorial process with no postal delays. When a conclusion is made about the publication of your paper by our Editorial Board, revisions can be submitted online with the same procedure, with an occasion to view and respond to all comments.

Complete support for both authors and co-author is provided.

\section{MANUSCRIPT'S CATEGORY}

Based on potential and nature, the manuscript can be categorized under the following heads:

Original research paper: Such papers are reports of high-level significant original research work.

Review papers: These are concise, significant but helpful and decisive topics for young researchers.

Research articles: These are handled with small investigation and applications

Research letters: The letters are small and concise comments on previously published matters.

\section{STRUCTURE AND FORMAT OF MANUSCRIPT}

The recommended size of original research paper is less than seven thousand words, review papers fewer than seven thousands words also.Preparation of research paper or how to write research paper, are major hurdle, while writing manuscript. The research articles and research letters should be fewer than three thousand words, the structure original research paper; sometime review paper should be as follows:

Papers: These are reports of significant research (typically less than 7000 words equivalent, including tables, figures, references), and comprise:

(a)Title should be relevant and commensurate with the theme of the paper.

(b) A brief Summary, "Abstract" (less than 150 words) containing the major results and conclusions.

(c) Up to ten keywords, that precisely identifies the paper's subject, purpose, and focus.

(d) An Introduction, giving necessary background excluding subheadings; objectives must be clearly declared.

(e) Resources and techniques with sufficient complete experimental details (wherever possible by reference) to permit repetition; sources of information must be given and numerical methods must be specified by reference, unless non-standard.

(f) Results should be presented concisely, by well-designed tables and/or figures; the same data may not be used in both; suitable statistical data should be given. All data must be obtained with attention to numerical detail in the planning stage. As reproduced design has been recognized to be important to experiments for a considerable time, the Editor has decided that any paper that appears not to have adequate numerical treatments of the data will be returned un-refereed;

(g) Discussion should cover the implications and consequences, not just recapitulating the results; conclusions should be summarizing.

(h) Brief Acknowledgements.

(i) References in the proper form.

Authors should very cautiously consider the preparation of papers to ensure that they communicate efficiently. Papers are much more likely to be accepted, if they are cautiously designed and laid out, contain few or no errors, are summarizing, and be conventional to the approach and instructions. They will in addition, be published with much less delays than those that require much technical and editorial correction. 
The Editorial Board reserves the right to make literary corrections and to make suggestions to improve briefness.

It is vital, that authors take care in submitting a manuscript that is written in simple language and adheres to published guidelines.

\section{Format}

Language: The language of publication is UK English. Authors, for whom English is a second language, must have their manuscript efficiently edited by an English-speaking person before submission to make sure that, the English is of high excellence. It is preferable, that manuscripts should be professionally edited.

Standard Usage, Abbreviations, and Units: Spelling and hyphenation should be conventional to The Concise Oxford English Dictionary. Statistics and measurements should at all times be given in figures, e.g. $16 \mathrm{~min}$, except for when the number begins a sentence. When the number does not refer to a unit of measurement it should be spelt in full unless, it is 160 or greater.

Abbreviations supposed to be used carefully. The abbreviated name or expression is supposed to be cited in full at first usage, followed by the conventional abbreviation in parentheses.

Metric SI units are supposed to generally be used excluding where they conflict with current practice or are confusing. For illustration, 1.4 I rather than $1.4 \times 10-3 \mathrm{~m} 3$, or $4 \mathrm{~mm}$ somewhat than $4 \times 10-3 \mathrm{~m}$. Chemical formula and solutions must identify the form used, e.g. anhydrous or hydrated, and the concentration must be in clearly defined units. Common species names should be followed by underlines at the first mention. For following use the generic name should be constricted to a single letter, if it is clear.

\section{Structure}

All manuscripts submitted to Global Journals Inc. (US), ought to include:

Title: The title page must carry an instructive title that reflects the content, a running title (less than 45 characters together with spaces), names of the authors and co-authors, and the place(s) wherever the work was carried out. The full postal address in addition with the email address of related author must be given. Up to eleven keywords or very brief phrases have to be given to help data retrieval, mining and indexing.

\section{Abstract, used in Original Papers and Reviews:}

Optimizing Abstract for Search Engines

Many researchers searching for information online will use search engines such as Google, Yahoo or similar. By optimizing your paper for search engines, you will amplify the chance of someone finding it. This in turn will make it more likely to be viewed and/or cited in a further work. Global Journals Inc. (US) have compiled these guidelines to facilitate you to maximize the web-friendliness of the most public part of your paper.

Key Words

A major linchpin in research work for the writing research paper is the keyword search, which one will employ to find both library and Internet resources.

One must be persistent and creative in using keywords. An effective keyword search requires a strategy and planning a list of possible keywords and phrases to try.

Search engines for most searches, use Boolean searching, which is somewhat different from Internet searches. The Boolean search uses "operators," words (and, or, not, and near) that enable you to expand or narrow your affords. Tips for research paper while preparing research paper are very helpful guideline of research paper.

Choice of key words is first tool of tips to write research paper. Research paper writing is an art.A few tips for deciding as strategically as possible about keyword search:

\section{(C) Copyright by Global Journals Inc.(US)| Guidelines Handbook}


- $\quad$ One should start brainstorming lists of possible keywords before even begin searching. Think about the most important concepts related to research work. Ask, "What words would a source have to include to be truly valuable in research paper?" Then consider synonyms for the important words.

- It may take the discovery of only one relevant paper to let steer in the right keyword direction because in most databases, the keywords under which a research paper is abstracted are listed with the paper.

- $\quad$ One should avoid outdated words.

Keywords are the key that opens a door to research work sources. Keyword searching is an art in which researcher's skills are bound to improve with experience and time.

Numerical Methods: Numerical methods used should be clear and, where appropriate, supported by references.

Acknowledgements: Please make these as concise as possible.

References

References follow the Harvard scheme of referencing. References in the text should cite the authors' names followed by the time of their publication, unless there are three or more authors when simply the first author's name is quoted followed by et al. unpublished work has to only be cited where necessary, and only in the text. Copies of references in press in other journals have to be supplied with submitted typescripts. It is necessary that all citations and references be carefully checked before submission, as mistakes or omissions will cause delays.

References to information on the World Wide Web can be given, but only if the information is available without charge to readers on an official site. Wikipedia and Similar websites are not allowed where anyone can change the information. Authors will be asked to make available electronic copies of the cited information for inclusion on the Global Journals Inc. (US) homepage at the judgment of the Editorial Board.

The Editorial Board and Global Journals Inc. (US) recommend that, citation of online-published papers and other material should be done via a DOI (digital object identifier). If an author cites anything, which does not have a DOI, they run the risk of the cited material not being noticeable.

The Editorial Board and Global Journals Inc. (US) recommend the use of a tool such as Reference Manager for reference management and formatting.

Tables, Figures and Figure Legends

Tables: Tables should be few in number, cautiously designed, uncrowned, and include only essential data. Each must have an Arabic number, e.g. Table 4, a self-explanatory caption and be on a separate sheet. Vertical lines should not be used.

Figures: Figures are supposed to be submitted as separate files. Always take in a citation in the text for each figure using Arabic numbers, e.g. Fig. 4. Artwork must be submitted online in electronic form by e-mailing them.

Preparation of Electronic Figures for Publication

Even though low quality images are sufficient for review purposes, print publication requires high quality images to prevent the final product being blurred or fuzzy. Submit (or e-mail) EPS (line art) or TIFF (halftone/photographs) files only. MS PowerPoint and Word Graphics are unsuitable for printed pictures. Do not use pixel-oriented software. Scans (TIFF only) should have a resolution of at least 350 dpi (halftone) or 700 to 1100 dpi (line drawings) in relation to the imitation size. Please give the data for figures in black and white or submit a Color Work Agreement Form. EPS files must be saved with fonts embedded (and with a TIFF preview, if possible).

For scanned images, the scanning resolution (at final image size) ought to be as follows to ensure good reproduction: line art: $>650$ dpi; halftones (including gel photographs) : >350 dpi; figures containing both halftone and line images: $>650 \mathrm{dpi}$. 
Color Charges: It is the rule of the Global Journals Inc. (US) for authors to pay the full cost for the reproduction of their color artwork. Hence, please note that, if there is color artwork in your manuscript when it is accepted for publication, we would require you to complete and return a color work agreement form before your paper can be published.

Figure Legends: Self-explanatory legends of all figures should be incorporated separately under the heading 'Legends to Figures'. In the full-text online edition of the journal, figure legends may possibly be truncated in abbreviated links to the full screen version. Therefore, the first 100 characters of any legend should notify the reader, about the key aspects of the figure.

\section{AFTER ACCEPTANCE}

Upon approval of a paper for publication, the manuscript will be forwarded to the dean, who is responsible for the publication of the Global Journals Inc. (US).

\subsection{Proof Corrections}

The corresponding author will receive an e-mail alert containing a link to a website or will be attached. A working e-mail address must therefore be provided for the related author.

Acrobat Reader will be required in order to read this file. This software can be downloaded

(Free of charge) from the following website:

www.adobe.com/products/acrobat/readstep2.html. This will facilitate the file to be opened, read on screen, and printed out in order for any corrections to be added. Further instructions will be sent with the proof.

Proofs must be returned to the dean at dean@globaljournals.org within three days of receipt.

As changes to proofs are costly, we inquire that you only correct typesetting errors. All illustrations are retained by the publisher. Please note that the authors are responsible for all statements made in their work, including changes made by the copy editor.

\subsection{Early View of Global Journals Inc. (US) (Publication Prior to Print)}

The Global Journals Inc. (US) are enclosed by our publishing's Early View service. Early View articles are complete full-text articles sent in advance of their publication. Early View articles are absolute and final. They have been completely reviewed, revised and edited for publication, and the authors' final corrections have been incorporated. Because they are in final form, no changes can be made after sending them. The nature of Early View articles means that they do not yet have volume, issue or page numbers, so Early View articles cannot be cited in the conventional way.

\subsection{Author Services}

Online production tracking is available for your article through Author Services. Author Services enables authors to track their article once it has been accepted - through the production process to publication online and in print. Authors can check the status of their articles online and choose to receive automated e-mails at key stages of production. The authors will receive an e-mail with a unique link that enables them to register and have their article automatically added to the system. Please ensure that a complete e-mail address is provided when submitting the manuscript.

\subsection{Author Material Archive Policy}

Please note that if not specifically requested, publisher will dispose off hardcopy \& electronic information submitted, after the two months of publication. If you require the return of any information submitted, please inform the Editorial Board or dean as soon as possible.

\subsection{Offprint and Extra Copies}

A PDF offprint of the online-published article will be provided free of charge to the related author, and may be distributed according to the Publisher's terms and conditions. Additional paper offprint may be ordered by emailing us at: editor@globaljournals.org . 
Before start writing a good quality Computer Science Research Paper, let us first understand what is Computer Science Research Paper? So, Computer Science Research Paper is the paper which is written by professionals or scientists who are associated to Computer Science and Information Technology, or doing research study in these areas. If you are novel to this field then you can consult about this field from your supervisor or guide.

\section{TECHNIQUES FOR WRITING A GOOD QUALITY RESEARCH PAPER:}

1. Choosing the topic: In most cases, the topic is searched by the interest of author but it can be also suggested by the guides. You can have several topics and then you can judge that in which topic or subject you are finding yourself most comfortable. This can be done by asking several questions to yourself, like Will I be able to carry our search in this area? Will I find all necessary recourses to accomplish the search? Will I be able to find all information in this field area? If the answer of these types of questions will be "Yes" then you can choose that topic. In most of the cases, you may have to conduct the surveys and have to visit several places because this field is related to Computer Science and Information Technology. Also, you may have to do a lot of work to find all rise and falls regarding the various data of that subject. Sometimes, detailed information plays a vital role, instead of short information.

2. Evaluators are human: First thing to remember that evaluators are also human being. They are not only meant for rejecting a paper. They are here to evaluate your paper. So, present your Best.

3. Think Like Evaluators: If you are in a confusion or getting demotivated that your paper will be accepted by evaluators or not, then think and try to evaluate your paper like an Evaluator. Try to understand that what an evaluator wants in your research paper and automatically you will have your answer.

4. Make blueprints of paper: The outline is the plan or framework that will help you to arrange your thoughts. It will make your paper logical. But remember that all points of your outline must be related to the topic you have chosen.

5. Ask your Guides: If you are having any difficulty in your research, then do not hesitate to share your difficulty to your guide (if you have any). They will surely help you out and resolve your doubts. If you can't clarify what exactly you require for your work then ask the supervisor to help you with the alternative. He might also provide you the list of essential readings.

6. Use of computer is recommended: As you are doing research in the field of Computer Science, then this point is quite obvious.

7. Use right software: Always use good quality software packages. If you are not capable to judge good software then you can lose quality of your paper unknowingly. There are various software programs available to help you, which you can get through Internet.

8. Use the Internet for help: An excellent start for your paper can be by using the Google. It is an excellent search engine, where you can have your doubts resolved. You may also read some answers for the frequent question how to write my research paper or find model research paper. From the internet library you can download books. If you have all required books make important reading selecting and analyzing the specified information. Then put together research paper sketch out.

9. Use and get big pictures: Always use encyclopedias, Wikipedia to get pictures so that you can go into the depth.

10. Bookmarks are useful: When you read any book or magazine, you generally use bookmarks, right! It is a good habit, which helps to not to lose your continuity. You should always use bookmarks while searching on Internet also, which will make your search easier.

11. Revise what you wrote: When you write anything, always read it, summarize it and then finalize it. 
12. Make all efforts: Make all efforts to mention what you are going to write in your paper. That means always have a good start. Try to mention everything in introduction, that what is the need of a particular research paper. Polish your work by good skill of writing and always give an evaluator, what he wants.

13. Have backups: When you are going to do any important thing like making research paper, you should always have backup copies of it either in your computer or in paper. This will help you to not to lose any of your important.

14. Produce good diagrams of your own: Always try to include good charts or diagrams in your paper to improve quality. Using several and unnecessary diagrams will degrade the quality of your paper by creating "hotchpotch." So always, try to make and include those diagrams, which are made by your own to improve readability and understandability of your paper.

15. Use of direct quotes: When you do research relevant to literature, history or current affairs then use of quotes become essential but if study is relevant to science then use of quotes is not preferable.

16. Use proper verb tense: Use proper verb tenses in your paper. Use past tense, to present those events that happened. Use present tense to indicate events that are going on. Use future tense to indicate future happening events. Use of improper and wrong tenses will confuse the evaluator. Avoid the sentences that are incomplete.

17. Never use online paper: If you are getting any paper on Internet, then never use it as your research paper because it might be possible that evaluator has already seen it or maybe it is outdated version.

18. Pick a good study spot: To do your research studies always try to pick a spot, which is quiet. Every spot is not for studies. Spot that suits you choose it and proceed further.

19. Know what you know: Always try to know, what you know by making objectives. Else, you will be confused and cannot achieve your target.

20. Use good quality grammar: Always use a good quality grammar and use words that will throw positive impact on evaluator. Use of good quality grammar does not mean to use tough words, that for each word the evaluator has to go through dictionary. Do not start sentence with a conjunction. Do not fragment sentences. Eliminate one-word sentences. Ignore passive voice. Do not ever use a big word when a diminutive one would suffice. Verbs have to be in agreement with their subjects. Prepositions are not expressions to finish sentences with. It is incorrect to ever divide an infinitive. Avoid clichés like the disease. Also, always shun irritating alliteration. Use language that is simple and straight forward. put together a neat summary.

21. Arrangement of information: Each section of the main body should start with an opening sentence and there should be a changeover at the end of the section. Give only valid and powerful arguments to your topic. You may also maintain your arguments with records.

22. Never start in last minute: Always start at right time and give enough time to research work. Leaving everything to the last minute will degrade your paper and spoil your work.

23. Multitasking in research is not good: Doing several things at the same time proves bad habit in case of research activity. Research is an area, where everything has a particular time slot. Divide your research work in parts and do particular part in particular time slot.

24. Never copy others' work: Never copy others' work and give it your name because if evaluator has seen it anywhere you will be in trouble.

25. Take proper rest and food: No matter how many hours you spend for your research activity, if you are not taking care of your health then all your efforts will be in vain. For a quality research, study is must, and this can be done by taking proper rest and food.

26. Go for seminars: Attend seminars if the topic is relevant to your research area. Utilize all your resources.

\section{(C) Copyright by Global Journals Inc.(US)| Guidelines Handbook}


27. Refresh your mind after intervals: Try to give rest to your mind by listening to soft music or by sleeping in intervals. This will also improve your memory.

28. Make colleagues: Always try to make colleagues. No matter how sharper or intelligent you are, if you make colleagues you can have several ideas, which will be helpful for your research.

29. Think technically: Always think technically. If anything happens, then search its reasons, its benefits, and demerits.

30. Think and then print: When you will go to print your paper, notice that tables are not be split, headings are not detached from their descriptions, and page sequence is maintained.

31. Adding unnecessary information: Do not add unnecessary information, like, I have used MS Excel to draw graph. Do not add irrelevant and inappropriate material. These all will create superfluous. Foreign terminology and phrases are not apropos. One should NEVER take a broad view. Analogy in script is like feathers on a snake. Not at all use a large word when a very small one would be sufficient. Use words properly, regardless of how others use them. Remove quotations. Puns are for kids, not grunt readers. Amplification is a billion times of inferior quality than sarcasm.

32. Never oversimplify everything: To add material in your research paper, never go for oversimplification. This will definitely irritate the evaluator. Be more or less specific. Also too, by no means, ever use rhythmic redundancies. Contractions aren't essential and shouldn't be there used. Comparisons are as terrible as clichés. Give up ampersands and abbreviations, and so on. Remove commas, that are, not necessary. Parenthetical words however should be together with this in commas. Understatement is all the time the complete best way to put onward earth-shaking thoughts. Give a detailed literary review.

33. Report concluded results: Use concluded results. From raw data, filter the results and then conclude your studies based on measurements and observations taken. Significant figures and appropriate number of decimal places should be used. Parenthetical remarks are prohibitive. Proofread carefully at final stage. In the end give outline to your arguments. Spot out perspectives of further study of this subject. Justify your conclusion by at the bottom of them with sufficient justifications and examples.

34. After conclusion: Once you have concluded your research, the next most important step is to present your findings. Presentation is extremely important as it is the definite medium though which your research is going to be in print to the rest of the crowd. Care should be taken to categorize your thoughts well and present them in a logical and neat manner. A good quality research paper format is essential because it serves to highlight your research paper and bring to light all necessary aspects in your research.

\section{INFORMAL GUIDELINES OF RESEARCH PAPER WRITING}

Key points to remember:

- Submit all work in its final form.

- Write your paper in the form, which is presented in the guidelines using the template.

- $\quad$ Please note the criterion for grading the final paper by peer-reviewers.

\section{Final Points:}

A purpose of organizing a research paper is to let people to interpret your effort selectively. The journal requires the following sections, submitted in the order listed, each section to start on a new page.

The introduction will be compiled from reference matter and will reflect the design processes or outline of basis that direct you to make study. As you will carry out the process of study, the method and process section will be constructed as like that. The result segment will show related statistics in nearly sequential order and will direct the reviewers next to the similar intellectual paths throughout the data that you took to carry out your study. The discussion section will provide understanding of the data and projections as to the implication of the results. The use of good quality references all through the paper will give the effort trustworthiness by representing an alertness of prior workings.

\section{(C) Copyright by Global Journals Inc.(US) | Guidelines Handbook}


Writing a research paper is not an easy job no matter how trouble-free the actual research or concept. Practice, excellent preparation, and controlled record keeping are the only means to make straightforward the progression.

\section{General style:}

Specific editorial column necessities for compliance of a manuscript will always take over from directions in these general guidelines.

To make a paper clear

- Adhere to recommended page limits

Mistakes to evade

- Insertion a title at the foot of a page with the subsequent text on the next page

- Separating a table/chart or figure - impound each figure/table to a single page

- Submitting a manuscript with pages out of sequence

In every sections of your document

• Use standard writing style including articles ("a", "the," etc.)

- Keep on paying attention on the research topic of the paper

- Use paragraphs to split each significant point (excluding for the abstract)

- Align the primary line of each section

- Present your points in sound order

- Use present tense to report well accepted

- Use past tense to describe specific results

- Shun familiar wording, don't address the reviewer directly, and don't use slang, slang language, or superlatives

- Shun use of extra pictures - include only those figures essential to presenting results

Title Page:

Choose a revealing title. It should be short. It should not have non-standard acronyms or abbreviations. It should not exceed two printed lines. It should include the name(s) and address (es) of all authors.

\section{(C) Copyright by Global Journals Inc.(US)| Guidelines Handbook}




\section{Abstract:}

The summary should be two hundred words or less. It should briefly and clearly explain the key findings reported in the manuscript-must have precise statistics. It should not have abnormal acronyms or abbreviations. It should be logical in itself. Shun citing references at this point.

An abstract is a brief distinct paragraph summary of finished work or work in development. In a minute or less a reviewer can be taught the foundation behind the study, common approach to the problem, relevant results, and significant conclusions or new questions.

Write your summary when your paper is completed because how can you write the summary of anything which is not yet written? Wealth of terminology is very essential in abstract. Yet, use comprehensive sentences and do not let go readability for briefness. You can maintain it succinct by phrasing sentences so that they provide more than lone rationale. The author can at this moment go straight to shortening the outcome. Sum up the study, with the subsequent elements in any summary. Try to maintain the initial two items to no more than one ruling each.

- Reason of the study - theory, overall issue, purpose

- Fundamental goal

- To the point depiction of the research

- Consequences, including definite statistics - if the consequences are quantitative in nature, account quantitative data; results of any numerical analysis should be reported

- $\quad$ Significant conclusions or questions that track from the research(es)

Approach:

- $\quad$ Single section, and succinct

- As a outline of job done, it is always written in past tense

- A conceptual should situate on its own, and not submit to any other part of the paper such as a form or table

- Center on shortening results - bound background information to a verdict or two, if completely necessary

- What you account in an conceptual must be regular with what you reported in the manuscript

- $\quad$ Exact spelling, clearness of sentences and phrases, and appropriate reporting of quantities (proper units, important statistics) are just as significant in an abstract as they are anywhere else

\section{Introduction:}

The Introduction should "introduce" the manuscript. The reviewer should be presented with sufficient background information to be capable to comprehend and calculate the purpose of your study without having to submit to other works. The basis for the study should be offered. Give most important references but shun difficult to make a comprehensive appraisal of the topic. In the introduction, describe the problem visibly. If the problem is not acknowledged in a logical, reasonable way, the reviewer will have no attention in your result. Speak in common terms about techniques used to explain the problem, if needed, but do not present any particulars about the protocols here. Following approach can create a valuable beginning:

- Explain the value (significance) of the study

- Shield the model - why did you employ this particular system or method? What is its compensation? You strength remark on its appropriateness from a abstract point of vision as well as point out sensible reasons for using it.

- Present a justification. Status your particular theory (es) or aim(s), and describe the logic that led you to choose them.

- Very for a short time explain the tentative propose and how it skilled the declared objectives.

Approach:

- Use past tense except for when referring to recognized facts. After all, the manuscript will be submitted after the entire job is done.

- Sort out your thoughts; manufacture one key point with every section. If you make the four points listed above, you will need a least of four paragraphs. 
- Present surroundings information only as desirable in order hold up a situation. The reviewer does not desire to read the whole thing you know about a topic.

- $\quad$ Shape the theory/purpose specifically - do not take a broad view.

- As always, give awareness to spelling, simplicity and correctness of sentences and phrases.

\section{Procedures (Methods and Materials):}

This part is supposed to be the easiest to carve if you have good skills. A sound written Procedures segment allows a capable scientist to replacement your results. Present precise information about your supplies. The suppliers and clarity of reagents can be helpful bits of information. Present methods in sequential order but linked methodologies can be grouped as a segment. Be concise when relating the protocols. Attempt for the least amount of information that would permit another capable scientist to spare your outcome but be cautious that vital information is integrated. The use of subheadings is suggested and ought to be synchronized with the results section. When a technique is used that has been well described in another object, mention the specific item describing a way but draw the basic principle while stating the situation. The purpose is to text all particular resources and broad procedures, so that another person may use some or all of the methods in one more study or referee the scientific value of your work. It is not to be a step by step report of the whole thing you did, nor is a methods section a set of orders.

Materials:

- Explain materials individually only if the study is so complex that it saves liberty this way.

- Embrace particular materials, and any tools or provisions that are not frequently found in laboratories.

- Do not take in frequently found.

- If use of a definite type of tools.

- Materials may be reported in a part section or else they may be recognized along with your measures.

Methods:

- $\quad$ Report the method (not particulars of each process that engaged the same methodology)

- Describe the method entirely

- To be succinct, present methods under headings dedicated to specific dealings or groups of measures

- Simplify - details how procedures were completed not how they were exclusively performed on a particular day.

- If well known procedures were used, account the procedure by name, possibly with reference, and that's all.

Approach:

- It is embarrassed or not possible to use vigorous voice when documenting methods with no using first person, which would focus the reviewer's interest on the researcher rather than the job. As a result when script up the methods most authors use third person passive voice.

- Use standard style in this and in every other part of the paper - avoid familiar lists, and use full sentences.

What to keep away from

- Resources and methods are not a set of information.

- $\quad$ Skip all descriptive information and surroundings - save it for the argument.

- Leave out information that is immaterial to a third party.

Results:

The principle of a results segment is to present and demonstrate your conclusion. Create this part a entirely objective details of the outcome, and save all understanding for the discussion.

The page length of this segment is set by the sum and types of data to be reported. Carry on to be to the point, by means of statistics and tables, if suitable, to present consequences most efficiently.You must obviously differentiate material that would usually be incorporated in a study editorial from any unprocessed data or additional appendix matter that would not be available. In fact, such matter should not be submitted at all except requested by the instructor.

\section{(C) Copyright by Global Journals Inc.(US) | Guidelines Handbook}


- Sum up your conclusion in text and demonstrate them, if suitable, with figures and tables.

- In manuscript, explain each of your consequences, point the reader to remarks that are most appropriate.

- Present a background, such as by describing the question that was addressed by creation an exacting study.

- Explain results of control experiments and comprise remarks that are not accessible in a prescribed figure or table, if appropriate.

- Examine your data, then prepare the analyzed (transformed) data in the form of a figure (graph), table, or in manuscript form. What to stay away from

- Do not discuss or infer your outcome, report surroundings information, or try to explain anything.

- Not at all, take in raw data or intermediate calculations in a research manuscript.

- Do not present the similar data more than once.

- Manuscript should complement any figures or tables, not duplicate the identical information.

- $\quad$ Never confuse figures with tables - there is a difference.

Approach

- $\quad$ As forever, use past tense when you submit to your results, and put the whole thing in a reasonable order.

- $\quad$ Put figures and tables, appropriately numbered, in order at the end of the report

- If you desire, you may place your figures and tables properly within the text of your results part.

Figures and tables

- If you put figures and tables at the end of the details, make certain that they are visibly distinguished from any attach appendix materials, such as raw facts

- Despite of position, each figure must be numbered one after the other and complete with subtitle

- In spite of position, each table must be titled, numbered one after the other and complete with heading

- All figure and table must be adequately complete that it could situate on its own, divide from text

Discussion

The Discussion is expected the trickiest segment to write and describe. A lot of papers submitted for journal are discarded based on problems with the Discussion. There is no head of state for how long a argument should be. Position your understanding of the outcome visibly to lead the reviewer through your conclusions, and then finish the paper with a summing up of the implication of the study. The purpose here is to offer an understanding of your results and hold up for all of your conclusions, using facts from your research and generally accepted information, if suitable. The implication of result should be visibly described. Infer your data in the conversation in suitable depth. This means that when you clarify an observable fact you must explain mechanisms that may account for the observation. If your results vary from your prospect, make clear why that may have happened. If your results agree, then explain the theory that the proof supported. It is never suitable to just state that the data approved with prospect, and let it drop at that.

- Make a decision if each premise is supported, discarded, or if you cannot make a conclusion with assurance. Do not just dismiss a study or part of a study as "uncertain."

- $\quad$ Research papers are not acknowledged if the work is imperfect. Draw what conclusions you can based upon the results that you have, and take care of the study as a finished work

- You may propose future guidelines, such as how the experiment might be personalized to accomplish a new idea.

- Give details all of your remarks as much as possible, focus on mechanisms.

- Make a decision if the tentative design sufficiently addressed the theory, and whether or not it was correctly restricted.

- Try to present substitute explanations if sensible alternatives be present.

- One research will not counter an overall question, so maintain the large picture in mind, where do you go next? The best studies unlock new avenues of study. What questions remain?

- Recommendations for detailed papers will offer supplementary suggestions.

Approach:

- When you refer to information, differentiate data generated by your own studies from available information

- Submit to work done by specific persons (including you) in past tense.

- Submit to generally acknowledged facts and main beliefs in present tense. 
Please carefully note down following rules and regulation before submitting your Research Paper to Global Journals Inc. (US):

Segment Draft and Final Research Paper: You have to strictly follow the template of research paper. If it is not done your paper may get rejected.

- The major constraint is that you must independently make all content, tables, graphs, and facts that are offered in the paper. You must write each part of the paper wholly on your own. The Peer-reviewers need to identify your own perceptive of the concepts in your own terms. NEVER extract straight from any foundation, and never rephrase someone else's analysis.

- $\quad$ Do not give permission to anyone else to "PROOFREAD" your manuscript.

- Methods to avoid Plagiarism is applied by us on every paper, if found guilty, you will be blacklisted by all of our collaborated research groups, your institution will be informed for this and strict legal actions will be taken immediately.)

- To guard yourself and others from possible illegal use please do not permit anyone right to use to your paper and files. 


\section{CRITERION FOR GRADING A RESEARCH PAPER (COMPILATION) \\ BY GLOBAL JOURNALS INC. (US)}

Please note that following table is only a Grading of "Paper Compilation" and not on "Performed/Stated Research" whose grading solely depends on Individual Assigned Peer Reviewer and Editorial Board Member. These can be available only on request and after decision of Paper. This report will be the property of Global Journals Inc. (US).

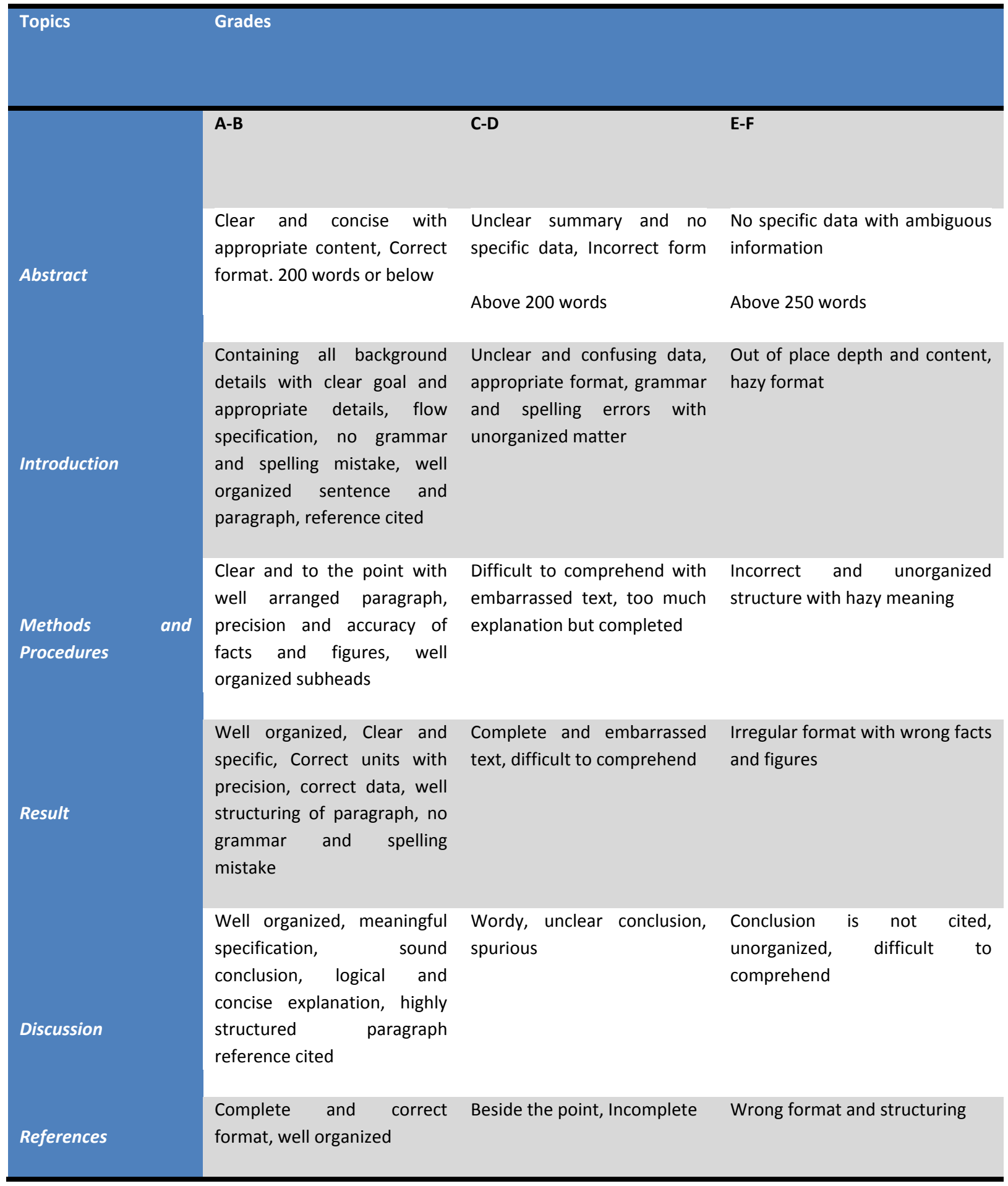




\section{INDEX}

\section{A}

Acoustics $\cdot 59$

Anomalies $\cdot 95$

B

Birefringent $\cdot 10$

c

Chalcogenide $\cdot 26$

Chaotic $\cdot 99,100,102,103$

$\bar{D}$

Dissipation · 104, 105, 107, 110, 116

$\bar{H}$

Hydraulics $\cdot 59$

I

Interferometric · 17, 19, 21

Iteration $\cdot 114$

\section{L}

Litharge $\cdot$ 2, 3, 25, 26

N

Neocortex $\cdot 110,112$

O

Onsager $\cdot 105$
$\bar{P}$

Penetrations $\cdot 63$

Perturbation · 9, 99, 100

$\boldsymbol{R}$

Redistribute $\cdot 17$

Regime $\cdot 6,9,110$

Resonator $\cdot 7,10$

\section{$S$}

Sapphire $\cdot 3,5,6,7,9,10,11$

Stagnation $\cdot 23$

Subluminal $\cdot 59$

$T$

Tardyon · 60, 61, 62, 63 


\section{Global Journal of Science Frontier Research}

Visit us on the Web at www.GlobalJournals.org | www.JournalofScience.org or email us at helpdesk@globaljournals.org 